the morphologic spectrum and etiology of DAD encountered during adult autopsy in an inner city teaching hospital. The diagnostic utility of post mortem lung culture was also evaluated.

Design: A retrospective study was performed on all adult autopsies from July 2010 to July 2013 with final histopathologic diagnosis of DAD. The histopathological features of DAD were re-evaluated by one autopsy pathologist and one pathology resident, based on the duration (exudative or proliferative phase), severity (bilateral/unilateral; focal/extensive) and pattern (classical vs. acute fibrinous and organizing pneumonia aka AFOP). Clinical history, pre and post mortem laboratory investigations, including postmortem lung culture (for bacteria, mycobacteria, fungi and virus) were reviewed to elucidate etiology.

Results: $36(16.2 \%)$ cases showed histopathologic features of DAD out of 222 adult autopsies in the three year study period. Clinical ARDS was documented in 20 of these cases $(55.6 \%)$. DAD was interpreted as the immediate cause of death in 19 cases $(8.6 \%)$ and described as bilateral and extensive distribution in $75 \%$ of cases. Morphologically, exudative phase and proliferative phase were found in 11 and 23 cases, respectively. AFOP pattern was identified in 2 cases. Infection ( 28 cases and $77.8 \%$ ) was the most common etiology for DAD (bacterial-10, fungal-4, viral-3, parasitic-1, undetermined-10). 6 patients had history of chronic interstitial lung disease with superimposed acute infection. Other causes included malignancy ( 3 cases) and chemotherapy related ( 1 case). Interestingly, even after exhaustive tissue cultures and medical record review, 4 cases (11.1\%) did not show any obvious cause of acute lung injury, raising the possibility of acute interstitial pneumonia (AIP). Post-mortem lung cultures were performed in 16 cases of which 9 cases $(56.3 \%)$ were positive ( 5 bacterial, 2 fungal and 2 viral infections).

Conclusions: Our study highlights the morphological spectrum of DAD encountered in adult hospital autopsy in an inner city teaching hospital, infection being the most common triggering factor, especially during acute exacerbation of chronic interstitial lung disease. A significant subpopulation of the cases had acute interstitial pneumonia, with no etiology identified. Post-mortem lung culture was a valuable diagnostic tool.

\section{Bone and Soft Tissue Pathology}

\section{Giant Cell Reparative Granuloma of Hands and Feet Show USP6 Gene Rearrangement: Are They Truly Solid Aneurysmal Bone Cysts?} NP Agaram, FV Le Loarer, L Zhang, S Hwang, EA Athanasian, $M$ Hameed, CR Antonescu. Memorial Sloan-Kettering Cancer Center, New York, NY.

Background: Giant cell reparative granulomas (GCRG) are lytic lesions of the bone that predominantly occur in the gnathic bones but have also been described in the small bones of the hands and feet. Morphologically, they are indistinguishable from the so-called 'solid variant' of Aneurysmal Bone Cysts (ABC) in extra-gnathic sites. The neoplastic nature of primary ABCs has been established with the identification of USP6 rearrangement in $70 \%$ of the cases. USP6 genetic alterations in giant-cell rich lesions (GCRG / ABC) of the small bones of the hands and feet has not been previously studied. Design: We investigated a group of 8 giant-cell rich lesions of the hands and feet by FISH for USP6 gene rearrangement, and further, compared the findings with other morphologically similar lesions including 9 gnathic GCRGs, 22 primary ABCs, 8 giant cell tumors of bone and 2 brown tumors of hyperparathyroidism.

Results: Overall, there were 49 samples from 48 patients including 26 females and 22 males. Radiologic imaging of the 8 lesions of the hands and feet showed 2 purely cystic, 1 purely solid and 4 mixed cystic and solid lesions. FISH for USP6 was performed on all of the 49 lesions in the study. Seven of the $8(88 \%)$ lesions of the hand and feet showed rearrangement of the USP6 gene. No USP6 gene rearrangements were identified in the 9 cases of gnathic GCRGs, 2 cases of brown tumor or the 8 cases of GCT of bone. Thirteen of the $22(59 \%)$ primary $\mathrm{ABCs}$ from the long bones and flat bones showed rearrangements of the USP 6 gene rearrangement.

Conclusions: Our results suggest that the majority of the GCRGs of the hands and feet represent true $\mathrm{ABCs}$ and should be reclassified as such. The terminology of GCRG should be restricted only to lesions in the gnathic location. FISH for USP6 is a useful ancillary tool in the diagnosis of primary $\mathrm{ABCs}$, and can be extremely helpful in distinguishing them from GCRGs and other morphologically similar lesions.

30 Extraskeletal Myxoid Chondrosarcoma with Non-EWSR1-NR4A3 Variant Fusions Correlate with Rhabdoid Phenotype and High Grade Morphology

NP Agaram, L Zhang, Y-S Sung, S Singer, CR Antonescu. Memorial Sloan-Kettering Cancer Center, New York, NY.

Background: Extraskeletal myxoid chondrosarcoma (EMC) is a rare soft tissue sarcoma with distinctive histology and uncertain histogenesis, characterized by EWSRI-NR4A3 fusion in $75 \%$ of the cases. A smaller proportion of cases show NR4A3 fused to other gene partners including TAF15, TCF 12 and TFG. The impact of various gene fusions on morphology and outcome has not been previously evaluated.

Design: We investigated a group of 26 consecutive EMCs with adequate material for FISH and/or RT-PCR analysis and correlated the genetic findings with morphology and clinical outcome.

Results: There were 5 females and 21 males, with a median age of 49.5 years. The mean size of the tumors was $11.1 \mathrm{~cm}$. FISH analysis showed that $16(62 \%)$ of the 26 cases had the EWSRI-NR4A3 gene fusion, 7 (27\%) cases showed TAF15-NR4A3 gene fusion and $1(4 \%)$ case showed TCF12-NR4A3 gene fusion. No rearrangements of the TFG or FUS genes were identified. Upon correlation, the morphology of most EWSRI-rearranged tumors ( 10 of 16) showed low cellularity, minimal cytologic atypia and low mitotic counts. In contrast, a predominant number of cases $(80 \%)$ with variant
(non-EWSR1) NR4A3 gene fusions (TAF15, TCF12) showed distinctive plasmacytoid / rhabdoid morphology, with increased cellularity, cytologic atypia and high mitotic counts. Follow-up showed that only 1 of 16 patients with EWSRI-rearranged tumors died of disease, in contrast to 3 of $7(43 \%)$ patients with TAF 15-rearranged tumors. Conclusions: In conclusion, EMCs with variant $N R 4 A 3$ gene fusions show a higher incidence of rhabdoid phenotype, high grade morphology and a more aggressive outcome compared to the more common EWSRI-NR4A3 positive tumors. Furthermore, as EWSR1 FISH break-apart assay is the preferred ancillary test to confirm diagnosis of EMC, tumors with variant $N R 4 A 3$ gene fusions remain under-recognized and often misdiagnosed. FISH assay for $N R 4 A 3$ rearrangements recognizes $>95 \%$ of EMCs and should be an additional tool in EWSRI-negative tumors.

31 Solitary Fibrous Tumor "Hemangiopericytoma" of Skin Is Rare; Other Differentials Should Be Considered

OI Ahmed, A AlSayyah, SS Qasem. Wake Forest School of Medicine, Winston Salem, NC.

Background: Solitary fibrous tumor (SFT), formerly hemangiopericytoma (HPC), is a rare neoplasm that can arise anywhere in the body. The majority of cases are benign; however, it can be of borderline or frank malignancy. Histologically it's composed of fibroblast-like spindled cells with variable cellularity, intervening collagen bundles and staghorn-like vasculature. Although the majority of SFT arise in the deep soft tissue, and body cavities, they can also be located superficially. In fact, they are frequently considered in the differential diagnosis of spindle cell lesions with prominent vasculature in the skin and subcutaneous tissue. The goal of this project is to study the frequency of SFT in skin.

Design: We searched our database for cases diagnosed as SFT or HPC. We then classified cases into superficial and deep based on the clinical information and the pathology report. The slides of all potential superficial cases were pulled and evaluated microscopically for the presence of skin in the specimen, the location of the tumor in relation to the skin and the histomorphology of the lesion. Any associated immunostains were also evaluated. Results: Our search retrieved 134 specimens, belonging to 108 patients, examined in our hospital over the course of 36 years. The specimens included 2 autopsies, 5 cytology specimens and 127 surgicals (biopsies and resections). $68 \%$ of the cases were considered benign, $4 \%$ were borderline and $28 \%$ were malignant. $81 \%$ percent were primary, $8 \%$ were recurrent and $11 \%$ were metastatic. The majority ( $87 \%$ ) were deep and only $13 \%$ were potentially superficial. Of the superficial cases, only 4 cases were involving or intimately associated with skin; 2 of which showed metastatic SFT from other deep preidentified primary sites; the other 2 were not morphologically conclusive for SFT (one is believed to be nodular fasciitis and the other is an aneurysmal fibrous histiocytoma).

Table 1

\begin{tabular}{|l|l|l||}
\hline Category & No. & \% \\
\hline Superficial & 17 & $13 \%$ \\
\hline Deep & 117 & $87 \%$ \\
\hline Benign & 91 & $68 \%$ \\
\hline Malignant & 37 & $28 \%$ \\
\hline Borderline & 6 & $4 \%$ \\
\hline Skin involved & 4 & $3 \%$ \\
\hline Primary & 108 & $81 \%$ \\
\hline Recurrent & 10 & $8 \%$ \\
\hline Metastatic & 16 & $11 \%$ \\
\hline
\end{tabular}

Conclusions: It is very rare to encounter SFT as a primary cutaneous neoplasm. There are several lesions in soft tissue with "HPC -like" architecture such as myofibroma, synovial sarcoma and fibrous histiocytoma. In addition CD34 staining is not specific for this tumor. Therefore, unless dealing with a patient with a known history of SFT, other differential diagnoses should be considered before making a diagnosis of SFT in the skin.

32 Novel ZC3H7B-BCOR and MEAF6-PHF1 Fusions in Ossifying Fibromyxoid Tumors - Molecular Characterization Shows Genetic Overlap with Endometrial Stromal Sarcoma

CR Antonescu, Y-S Sung, C-L Chen, L Zhang, H-W Chen, NP Agaram, CD Fletcher. Memorial Sloan-Kettering Cancer Center, New York, NY; Brigham and Women's Hospital, Boston, MA.

Background: $P H F 1$ gene rearrangements have been recently described in around $50 \%$ of ossifying fibromyxoid tumors (OFMT) including benign and malignant cases, with a small subset showing $E P 400-P H F 1$ fusions. In the remaining cases no alternative gene fusions have been identified. PHFl-negative OFTs, especially if lacking S100 protein staining or peripheral ossification, are difficult to diagnose and distinguished from other soft tissue mimics.

Design: In seeking more comprehensive molecular characterization, we investigated a large cohort of 39 OFMT of various anatomic sites, immunoprofiles and grades of malignancy. Tumors were screened for PHF1 and EP4OO rearrangements by FISH. RNA sequencing was performed in two index cases (OFMT1, OFMT3), negative for EP400-PHF1 fusions, followed by FusionSeq data analysis, a modular computational tool developed to discover gene fusions from paired-end RNA-seq data.

Results: Two novel fusions were identified $Z C 3 H 7 B-B C O R$ in OFMT1 and MEAF6$P H F 1$ in OFMT3. After being validated by FISH and RT-PCR, these abnormalities were screened on the remaining cases. With these additional gene fusions, the majority $(85 \%)$ of OFMTs with classic morphologic appearance demonstrated recurrent gene rearrangements, regardless of degree of malignancy, presence of ossification or immunoprofile, which can be used as molecular markers in challenging cases. The most common abnormality is $P H F 1$ gene rearrangement ( $80 \%$ ), being present in benign, atypical and malignant lesions, with fusion to EP400 in $44 \%$ of cases.

Conclusions: $Z C 3 H 7 B-B C O R$ and $M E A F 6-P H F 1$ fusions occurred predominantly in S100 protein-negative and malignant OFMT. Similar gene fusions have been reported in endometrial stromal sarcoma (ESS), a tumor seemingly unrelated to 
OFMT. Furthermore, similar with ESS pathogenesis, it appears that translocation genes involved in acetylation (MEAF6) and methylation $(P H F 1)$ have a role in the neoplastic development of OFMT.

\section{Diagnostic Pitfalls of Wilms Tumor (WT) Versus Desmoplastic Small Round Cell Tumor (DSRCT): Overlapping Morphologic and} Immunohistochemical Features

CA Arnold, L Schoenfield, MA Arnold. The Ohio State University, College of Medicine, Columbus, OH; Nationwide Children's Hospital, Columbus, $\mathrm{OH}$.

Background: Blastemal predominant Wilms Tumor (WT) and Desmoplastic Small Round Cell Tumor (DSRCT) have overlapping histologic features, yet accurate distinction is critical because of differing clinical management and prognostic significance. We recently encountered a case of blastemal predominant WT in a neck mass of a young adult who also had an abdominal mass. The neck mass was diagnosed as DSRCT based on a desmoplastic stroma, small round blue cell morphology, and reactivity for desmin (perinuclear accentuation), and cytokeratins. However, triphasic elements were identified on deeper sections and EWSRI rearrangement studies were negative; supporting the ultimate diagnosis of WT. Based on this challenging case, we undertook a comparison study of desmin and cytokeratin reactivity in WT and DSRCT. Design: Since the blastema of WT can mimic DSRCT, we compared the reactivity patterns of the blastemal components of WT to DSRCT. Twenty two WT resections (triphasic $=14$, blastemal $=8$ ) and 12 DSRCT (including 10 cases from 7 patients with confirmatory molecular studies) were retrospectively reviewed. H\&E sections were compared to desmin, and CAM5.2 and/or AE1/3 immunohistochemical stains and scored by 3 pathologists.

Results: Desmin reactivity was seen in 11/22 WT blastema and 11/12 DSRCT. While diffuse desmin reactivity was limited to DSRCT (0/11 WT vs. 5/11 DSRCT), dot-like or perinuclear accentuation was seen in nearly all desmin positive cases of both WT and DSRCT (10/11 WT, 10/11 DSRCT); larger discrete dots were more common in DSRCT. Cytoplasmic cytokeratin reactivity was seen in nearly all cases $(21 / 22 \mathrm{WT}$ blastema, 10/12 DSRCT). Cytokeratin staining of WT blastema was more often diffuse compared to DSRCT (11/21 WT vs 3/10 DSRCT).

Conclusions: Herein, we report a comparison study of desmin and cytokeratin reactivity patterns in WT and DSRCT, which shows marked overlap. Moreover, although dotlike desmin reactivity is traditionally associated with DSRCT, we demonstrate that nearly all desmin positive cases of WT blastema displayed dot-like or perinuclear desmin accentuation; serving as an important diagnostic pitfall. This distinction is most challenging in blastemal predominant WT, particularly in metastatic sites with associated desmoplasia. In these cases, detection of EWSRI rearrangement (seen with DSRCT) and immunoreactivity with antibodies to the amino-terminus of WT1 (seen in WT) remain the most reliable diagnostic tools.

\section{Deep Myxomas: Reappraisal of Desmin Expression and Correlation} with Novel Genetic Finding

$R$ Berry, T Bocklage. University of New Mexico SOM, Albuquerque, NM.

Background: Deep myxomas (juxta-articular [J-A] and intramuscular [IM]) have been described to variably express desmin. While the 2013 W.H.O. Atlas affirms this for IM myxoma, it provides no data regarding desmin expession in J-A myxoma. However, other new soft tissue tumor texts state that desmin expression is absent in IM myxoma. We reviewed recent cases to determine desmin reactivity in deep myxomas evaluating whether desmin reactive tumors could be distinguished clinically, histologically, or genetically from non-desmin-reactive myxomas.

Design: Twenty six deep myxomas were identified in Pathology with slides and blocks available. Patients were adults and comprised 13 males and 13 females with an age range of 40-88 years (mean 59 years). Tumors were located in extremity deep tissues. Imaging studies and follow-up was available for 24 cases. Immunohistochemistry was performed on one representative whole tumor section per case using a Dako immunostainer and a Dako desmin monoclonal antibody (clone D33 diluted 1:100) after antigen retrieval using Dako Hi pH Target Retrieval Solution in a pressure cooker decloaker for 20 minutes. Slides were incubated 30 minutes at room temperature. Re-review of clinical history, imaging studies, karyotypes $(\mathrm{n}=16)$ and all slides was performed. Desmin expression was assessed as percent reactivity and intensity.

Results:

Deep Myxomas: Desmin Reactivity and Genetics

\begin{tabular}{|c|c|c|c|c|}
\hline Feature & Desmin Positive & $\begin{array}{l}\begin{array}{l}\text { Desmin } \\
\text { Negative }\end{array} \\
\end{array}$ & $\begin{array}{l}\text { Karyotype } \\
\text { Normal }\end{array}$ & $\begin{array}{l}\text { Karyotype } \\
\text { Abnormal }\end{array}$ \\
\hline Classic Myxoma & 6 & 10 & 7 & 3 \\
\hline Cellular Myxoma & 2 & 3 & 2 & 1 \\
\hline Juxta-articular Myxoma & 3 & 2 & 1 & 2 \\
\hline Totals (\%) & $11(42 \%)$ & $15(58 \%)$ & $10(63 \%)$ & $6(37 \%)$ \\
\hline
\end{tabular}

10/16 IM myxomas karyotyped; 3/5 cellular myxomas karyotyped; 3/5 J-A myxomas karyotyped Eleven of 26 cases ( $42 \%$ ) expressed desmin with three strongly in $>40 \%$ of cells. Three cases with diffuse expression shared aberrations of 17q21-22 by karyotyping (one not karyotyped). No other features distinguished these tumors from the other myxomas.

Three Deep Myxomas with High Desmin Expression and Shared Changes of 17q21-22

\begin{tabular}{|l|l|l||}
\hline Case Diagnosis & Desmin Expression & Karyotype \\
\hline Intramuscular Myxoma & $40 \%$ & $\mathrm{t}(3 ; 17)(\mathrm{q} 27 ; \mathrm{q} 21) ;$ del17(q21q25) and others \\
\hline Cellular myxoma & $75 \%$ & $\mathrm{t}(9 ; 17)(\mathrm{p} 21 ; \mathrm{q} 22)$ and others \\
\hline Juxta-articular myxoma & $15 \%$ & add(17)(q21) and others \\
\hline \hline
\end{tabular}

desmin intensity: $3+$; other positive cases $1-5 \%$

Conclusions: A sizable minority of deep myxomas express desmin. Those with strong expression appear to be genetically distinct from other deep myxomas. However, behavior and clinical characteristics of these greater desmin positive tumors appear to match those of other deep myxomas. Review of more cases and molecular analyses are ongoing.
35 Head and Neck and Meningeal Hemangiopericytomas Are a Histological Variant of Solitary Fibrous Tumors

$M$ Biscuola, $R$ Avila, B Vieites, MA Lopez-Garcia, D Marcilla, F Carmona, A Blanco, $R$ Alvarez, F Sanchez, E de Alava. Virgen del Rocío Hospital, Seville, Spain; Miguel Servet Hospital, Zaragoza, Spain.

Background: Soft Tissue Hemangiopericytoma (HPC) and Solitary Fibrous Tumor (SFT) were considered by pathologists as two variants of a single tumor entity until were reclassified only as SFTs due to the detection of novel markers like GRIA2 and STAT6 overexpression and principally the molecular fusion occurring between NAB2 and STAT6 genes. Our hypothesis is that HPCs and SFTs could be considered the same entity regardless of the anatomical localization and to elucidate this point we tested these markers on a series of Head\&Neck, Meningeal and Soft Tissue HPC/SF tumours. Design: Samples from $44 \mathrm{HPC} / \mathrm{SFTs}$ (11 Meningeal, 5 Head\&Neck and 28 S.T. tumours) were obtained from Pathology Departments. Representative areas were carefully selected from stained sections of each tumour and $1-\mathrm{mm}$ diameter tissue cores were obtained. Immunohistochemistry was carried out on sequential TMA sections using CD34, SMA, $\mathrm{Bcl} 2$, EMA, Ki67 to confirm the previous histological diagnoses; GRIA2 and STAT6 as novel markers. In order to detect the possible rearrangements of the NAB2-STAT6 fusion and the correlation of this alteration with the nuclear reallocation of STAT6, specific RT-PCR primers for the two more common rearrangements were tested on the 53 samples after RNA isolation.

Results: We observed positive staining for CD34 and Ki-61 in the $86 \%$ of the cases, for SMA in the $27 \%$, for $\mathrm{Bcl} 2$ in the $50 \%$ and for EMA in the $16 \%$ of the samples. Over expression of GRIA2 was observed in the $42.9 \%$ of the cases, being positive in $20 \%$ of HPCs and in $20.5 \%$ SFTs. Nuclear expression of STAT 6 was positive in the $78.5 \%$ of the samples, $31.8 \%$ of HPCs and in $38.6 \%$ SFTs respectively. No staining was observed in negative control cases. We obtained positive RT-PCR amplification for the Exon4-Exon3 NAB2-STAT6 rearrangement in the 13\% of the cases. For those cases, we observed positive correlation with nuclear STAT6 expression in 5 out 6 of them. Unfortunately no samples showed the Exon6-Exon17/18/19 rearrangement, probably due to degradation of nucleic acid obtained from the FFPE tissues and the expected length of PCR amplification products.

Conclusions: Our results based on the GRIA2 and STAT6 expression suggest that HPCs and SFTs could be considered as one entity regardless of the anatomical localization. Specifically HPCs of the meningeal area behave like their soft tissue counterpart, indicating that they could belong to the SFT group. Due to the short amount of cases, HPCs of the Head and Neck tract require further studies to reach definitive conclusions.

36 Expression of p16 in Hibernomas - A Pitfall in Differentiating Adipocytic Tumors

MD Cain, GP Siegal, S Wei. University of Alabama at Birmingham, Birmingham, AL. Background: Hibernoma is a rare adipocytic tumor composed of brown fat. It occurs predominantly in young adults but has a wide age distribution and may rarely involve elderly patients. While the tumor normally does not present a diagnostic dilemma in a large excision, it can be challenging on a small biopsy as some of the neoplastic cells have transparent cytoplasm with multivacuolation, thus mimicing the lipoblasts commonly present in atypical lipomatous tumor/well-differentiated liposarcoma (ALT/ WDL). Distinguishing these entities is important for prognosis as excision is curative in hibernomas with no significant potential for recurrence. Recently, studies have shown that MDM2, CDK4 and p16 can be used as an adjunct to distinguish lipoma from WDL. Limited previous data have showed that about $50 \%$ of hibernomas immunoreacted with MDM2 and none expressed CDK4 and p16. In this study, we sought to further explore the specificity of CDK4 or p16 in distinguishing hibernoma from WDL. Moreover, given that all three molecules are closely related to p53 and that the aberrant MDM2+/ $\mathrm{p} 53+$ profile is a frequent phenotype in WDL, the expression of $\mathrm{p} 53$ was also examined. Design: The surgical pathology database of the authors' institution was searched to identify hibernoma cases. Immunohistochemical stains for CDK4, p16 and p53 were performed. Unequivocal nuclear staining was regarded as positive and was further ranked $1+(1-10 \%), 2+(11-50 \%)$ or $3+(>50 \%)$.

Results: Of 12 consecutive hibernoma cases retrieved, 7 (58\%) demonstrated nuclear reactivity for p16, including 6 with $1+$ and 1 with $2+$ staining. One case showed $1+$ staining for CDK4. All cases were negative for p53. Interestingly, this panel was also performed on a separate case of retroperitoneal WDL with prominent hibernomatous differentiation, which revealed the same p16+/CDK4-/p53-immunoprofile.

Conclusions: In contrast to a single previous study, p16 expression was seen in most hibernoma cases; therefore, it is not a promising marker in distinguishing hibernoma from WDL. In keeping with the prior findings, CDK4 is a relatively useful biomarker in this setting although it is not entirely specific. Further, p53 overexpression was not seen in any of the hibernoma cases examined, thus it can be utilized as an adjunct to other markers in the differential diagnosis. Given that MDM2 expression is reportedly seen in $50 \%$ of hibernomas, in situ hybridization analysis of MDM2 in these cases is ongoing. Large-scale studies to include other special types of benign adipocytic tumors are needed to investigate the utility of this "adipocytic tumor panel" in pathology practice.

37 Period

MD Cain, GP Siegal, S Wei. University of Alabama at Birmingham, Birmingham, AL. Background: The skeleton is a common site for tumor metastasis. Over 400,000 patients are diagnosed with bone metastasis (BM) annually in the US. It has long been accepted that osteotropism is an intrinsic property of certain malignances, with carcinomas of the breast $(\mathrm{BC})$, prostate $(\mathrm{PC})$ and lung $(\mathrm{LC})$ reportedly being the most prevalent primaries in general population. The highest incidences of BM have been seen in patients with these as well as thyroid and renal cell (RCC) carcinomas in postmortem examinations. 
However, it has been unclear whether these trends have remained constant over time. The aim of the study was to examine the frequencies of primary tumors among all patients with bone metastases over the last $3+$ decades.

Design: The tumor registry of the authors' institution was searched to identify cases of BM. The clinicopathological characteristics were recorded. Hematologic malignancies and soft tissue sarcomas directly involving bone were excluded. The remaining cases were divided into decades and further separated by gender. $\mathrm{X}^{2}$ test was utilized for statistical analysis.

Results: There were 2,355 cases identified by our inclusion criteria. LC was the most frequent primary tumor in men across all decades, followed by PC (ranged $45-50 \%$ vs. $18-23 \%$; $<.0001)$. While $\mathrm{BC}$ and $\mathrm{LC}$ showed similar frequencies in women in the 1980 's (33\% and 32\%, respectively), lung surpassed breast as the primary tumor site by approximately $10 \%$ thereafter in each decade $(37-41 \%$ vs. $26-34 \%, \mathrm{P}=.03)$. The other most common tumors included RCC, head/neck (H\&N; excluding thyroid) and gastrointestinal carcinomas in men; and those of $H \& \mathrm{~N}, \mathrm{RCC}$ and gynecologic origins in women, accounting for about $15 \%$ in each gender. No tumor type showed a significant variation in frequency over the study period. Tumors of unknown primary (TUP) dropped significantly from $14 \%$ (1980's) to $7 \%$ (1990's) to $0.6 \%$ (2000 and thereafter; $\mathrm{P}<0.001$ ), with no difference between genders. Not surprisingly, PC showed the highest tendency to relapse in bone on the first metastasis (95\%), followed by H\&N (93\%), LC $(82 \%), \mathrm{BC}(79 \%)$ and RCC $(71 \%)$

Conclusions: The trends in primary tumor metastasizing to bone have remained consistent over the last few decades. Given that $\mathrm{PC}$ and $\mathrm{BC}$ are by far the most prevalent malignancies in men and women, respectively, LC represents the highest number of metastatic bone disease cases relative to its incidence. These changes may represent locoregional predisposing factors to $\mathrm{LC}$ or later detection when compared to $\mathrm{PC}$ and $\mathrm{BC}$. The significant drop in TUP reflects the recent advancement in immunohistochemistry and molecular genetic techniques.

\section{$38 \quad$ GIST Associated with Desmoid Fibromatosis}

$M$ Caragea, D Stockman, K Torres, A Lazar, J Meis. MD Anderson Cancer Center, Houston, TX

Background: Gastrointestinal stromal tumors (GIST) may be associated with other neoplasms (carcinoma, lymphoma, leukemia) as either a preceding or synchronous malignancy. The association of GIST and mesenchymal neoplasms is relatively rare (neurofibroma, liposarcoma and leiomyosarcoma). We report 8 patients with GIST who also had desmoid fibromatosis in close anatomic proximity.

Design: Patients with GIST associated with desmoid fibromatosis were retrieved from the files of a major referral center. Morphologic, immunohistochemical, sequencing, and outcome data were analyzed.

Results: Eight patients with desmoid fibromatosis and GIST were identified. All tumors occurred within the abdominal cavity. Median patient age was 59 years with males predominating (6/8). GIST occurred prior to the development of desmoid in 6 patients and at the site of the prior surgical resection in 3 . In one patient the tumors were synchronous and in one the desmoid preceded GIST. Median interval to the development of the $2^{\text {nd }}$ tumor was 36 mos. Most GIST occurred in the stomach (5), followed by small bowel (2) and omentum (1). Median GIST size was $7.5 \mathrm{~cm}$. Six GIST were categorized as moderate to high risk and 2 as low risk. KIT and/or PDGFRA mutation analyses (5) revealed point mutations or deletions in exon 11 of the $K I T$ gene. Desmoids ranged from $1.5-8.5 \mathrm{~cm}$ and were located in gastric and perigastric soft tissue (3), small bowel (2), colon (1) and abdominal wall (1). Mutations were detected in exon 3 of the CTNNB1 gene (5). Clinical follow-up in 7 patients revealed 5 were alive and disease free and 2 were alive with disease (one with desmoid and one with GIST). Two patients with GIST developed recurrences and one hepatic metastasis.

Conclusions: The occurrence of GIST with desmoid fibromatosis does not appear to be fortuitous based on the cases in this series and the small number of reported cases. Notably, all cases of GIST in this series were intra-abdominal as were the desmoids, with nearly $40 \%$ of desmoids occurring at the site of a GIST that had been surgically removed. No relationship was found between the specific mutations seen in the GISTs or desmoids. A recurrent mass at the site of a previous GIST should not be assumed to be GIST clinically and vice versa. Histologic confirmation with appropriate immunohistochemical and molecular studies is strongly recommended in such cases.

$39 \quad$ Atypical Neurofibromata Do Not Display Malignant Behavior Despite Concerning Histologic Features

$M$ Caragea, A Lazar, W-L Wang. MD Anderson Cancer Center, Houston, TX.

Background: Atypical neurofibromata are usually seen in the setting of neurofibromatosis. They can be clinically and histiologically concerning with increased cellularity, scattered atypia and rare mitotic activity. While the criteria for diagnosis are not well established, there are certain peripheral nerve sheath tumors that warrant such diagnosis. We assessed the clinical behavior and outcome of these lesions at our institution.

Design: Twenty-four cases (20 patients) were retrieved from pathology archives. Available clinical data and tumor properties were tabulated. The tumors were evaluated for the following: growth pattern, cellularity, cytologic atypia, mitotic count and necrosis. Immunohistochemistry for $\mathrm{S} 100$ protein, $\mathrm{P} 53$ and $\mathrm{Ki}-67$ were performed.

Results: The median age was 21 years-old (range: 15 and 68) with equal male:female ratio. Tumors arose primarily in the setting of neurofibromatosis type $1(14 / 24)$ or in association with nerves $(6 / 24)$. Most involved the trunk and extremities with a median size of 5.5 (range: 1.1 to 9.5 ) $\mathrm{cm}$. Most patients presented with enlarging mass sometimes with compressive symptoms, such as pain or numbness, or in the setting of known neurofibromatosis complicated by PET avid tumors (20/24). Surgical resection was performed in 22/24 cases. Microscopically, all tumors were composed of spindle cells with wavy nuclei with a classic neurofibromatous growth pattern, some with myxoid stroma. The punctuated cytologic atypia was scattered throughout the neoplasm, ranging from moderate to scattered highly atypical nuclei $(n=21)$. Most $(n=14)$ showed $1-2$ mitoses/10 HPF. Necrosis was not identified. S-100 protein was performed in 12/24 cases and was diffusely and strongly expressed in all, while P53 was negative or weakly expressed in 10/24 cases (5-40\% of tumor nuclei). Ki- 67 marked $<1-30 \%$ of tumor nuclei. Of patient with follow-up ( $n=16)$, one progressed to MPNST in the same location one year after excision. Most patients are alive (12), disease free or living with disease. Four patients died, three of unknown cause (but not related to aggressive tumor behavior) and one due to surgical complications.

Conclusions: Atypical neurofibromas represent a subset of peripheral nerve sheath tumors seen primarily in the setting of neurofibromatosis. Despite their concerning cytologic and architectural features, these tumors behave in a benign fashion and should be distinguished from MPNST. Progression to MPNST appears to be rare. S100 protein is diffusely expressed in atypical neurofibromas and is helpful in this differential diagnosis.

\section{Malignant Peripheral Nerve Sheath Tumor (MPNST) with Low Grade Histologic Features Is a Fully Malignant Neoplasm with Aggressive} Behavior

$M$ Caragea, A Lazar, W-L Wang. MD Anderson Cancer Center, Houston, TX.

Background: MPNST with low grade histologic features is controversial with some doubting the utility of histologic grading. However, some neurogenic sarcomas with increased cellularity and mitotic activity lack the overt atypia and necrosis seen in most conventional MPNST. We examined a cohort of patients with tumors which fall within the spectrum of MPNST but with low-grade histology.

Design: Cases were obtained from our pathology archives. Association with large nerves or neurofibromatosis was noted. Criteria used for diagnosis include: increased cellularity, fascicular growth, uniform cellular histology with relatively mild atypia, mitotic activity, and patchy S100 protein expression. Clinical data and tumor properties were tabulated. The tumors were evaluated for: growth pattern, cellularity, cytologic atypia, mitotic count and necrosis. A panel of immunohistochemical markers, including S-100 protein, p53 and Ki-67 was performed.

Results: Eight cases were retrieved. Majority were sporadic (6/8) while 2 patients had NF1. The median age was 40 (range $16-64$ ) years-old. Majority of patients were females $(5 / 8)$. Most presented with an enlarging mass and involved the upper extremity and head and neck neural plexus. Median tumor size was $7 \mathrm{~cm}$ (range 1.2 to 9.5 ) $\mathrm{cm}$. Most tumors (5/8) displayed a fascicular growth pattern with fibrous bands separating nodules with a vague alternating hypo- and hypercellular areas and sometimes peripheral condensation. A myxoid background was appreciated in some tumors. The neoplastic cells were monotonous, spindled to more rounded, with relatively mild atypia. Median mitotic activity $3 / 10 \mathrm{hpf}$ (range: 1 to 10 mitoses per 10 high power fields). Necrosis was not identified. $5 / 8$ cases had patchy labeling for S-100 protein. Weak nuclear P53 was expressed in $3 / 4$ case and strong in 1(range, $15-80 \%$ of nuclei). The Ki- 67 proliferative index was low, with median of $5 \%$ (range $<1 \%-30 \%$ ). Seven patients had follow up with a median follow up time of 2 years (range 1-6 years). Local recurrence was seen in $50 \%(4 / 8)$ of the patients, while metastases were documented in one case. Four patients succumbed: 2 with disease (median time to death 3 years) and two of unknown causes. One patient is well with no evidence of disease.

Conclusions: Neurogenic sarcomas can have distinctive low-grade morphology that is different from atypical neurofibromas and classical high grade MPNST, and most are sporadic. Despite their low grade histologic features, are aggressive neoplasms with high rates of local recurrence and metastatic capacity.

41 Atypical Notochordal Cell Tumors: A Clinicopathologic Review of "Unclassifiable" Notochordal Tumors

JM Carter, DE Wenger, CY Inwards. Mayo Clinic, Rochester, MN.

Background: By current WHO classification, notochordal-derived tumors include benign notocordal cell tumor (BNCT) and chordoma, a malignant tumor with locally aggressive behavior. Defined radiologic and histologic criteria distinguish them. For example, BNCTs do not show radiologic evidence of bone destruction or extra-osseous extension. This distinction is essential as chordomas warrant aggressive surgical management while BNCT can be followed with serial imaging. We report our experience with a subset of BNCTs with atypical features and propose an alternative category of atypical notochordal cell tumors.

Design: Three cases of BNCTs with unusual radiologic features were retrieved from our archives. Radiologic re-review was performed to assess cortical and soft tissue involvement. Histologic re-review was performed. Immunohistochemistry for cytokeratins and brachyury was performed.

Results: Case 1 ( 52 y, male): MRI showed a $2.1 \mathrm{~cm}$ sacral (S4) lesion showing features of BNCT with the exception of cortical breakthrough with minimal soft tissue extension. Following complete resection, histologically, the tumor (in toto) showed typical features of BNCT. At 36 mos follow-up, there is no evidence of recurrent disease. Case 2 (60 $y$, male): MRI demonstrated a lumbar (L4) spine lesion showing radiologic features of BNCT with the exception of a minimal $(6.0 \mathrm{~mm})$ extradural soft tissue mass. Histologically, the biopsy tissue was BNCT. Re-biopsy at 6 yrs showed histologic features of BNCT with very focal myxoid change and tumor cell cording. However, serial imaging (total $8 \mathrm{yrs}$ ) has shown $<2 \mathrm{~mm}$ interval growth of the soft tissue component. He continues to be followed with serial imaging. Case 3 ( $74 \mathrm{y}$, male): MRI demonstrated a sacral mass (S3) showing features of BNCT with the exception of cortical breakthrough posteriorly and minimal extension into the sacral canal. Histologically, the biopsy tissue was BNCT. To date ( 8 mos), there is no evidence of tumor progression. In all cases, the tumors expressed cytokeratins and brachyury. 
Conclusions: Our 3 cases do not fit the current diagnostic criteria for BNCT or chordoma. To date, we have no evidence to support the need for aggressive surgery in this setting. Thus we propose an alternative diagnostic term, "atypical notochordal cell tumor", for this tumor subset. Additional cases with longer follow-up will be necessary to fully understand the biology of these unusual tumors.

\section{Chromogenic In Situ Hybridization for FGF23 mRNA in the Diagnosis of Phosphaturic Mesenchymal Tumors}

JM Carter, A Dogan, BL Caron, AL Folpe. Mayo Clinic, Rochester, MN; Memorial Sloan-Kettering Cancer Center, New York, NY.

Background: Phosphaturic mesenchymal tumors (PMT) are extraordinarily rare mesenchymal tumors, which can cause tumor-induced osteomalacia (TIO) through production of phosphaturic hormones, most often fibroblast growth factor-23 (FGF23). Distinction of PMT from morphologic mimics is important, as complete resection may be curative of debilitating TIO. Detection of $F G F 23$ expression is a valuable diagnostic adjunct, especially in patients with occult TIO. However, commercial FGF23 antibodies lack specificity, and RT-PCR for $F G F 23$ mRNA is excessively sensitive, detecting low levels of endogenous $F G F 23$ mRNA in normal bone. For these reasons, we developed a novel chromogenic in situ hybridization assay for $F G F 23$ mRNA.

Design: Cases of PMT (14) and histologic mimics (20) including chondromyxoid fibroma, chondroblastoma, osteoblastoma, and various soft tissue tumors were retrieved from our archives. In situ hybridization for $F G F 23$ mRNA was performed in archival tissues using the RNAscope ${ }^{\circledR}$ 2.0 FFPE Assay (ACD Inc. Hayward, CA) with appropriate control probes. Using a HRP-based signal amplification system, punctate intracellular staining was scored as "positive" using a semi-quantitative scoring method (3+: >10 signals/cell; 2+: 4-10 signals/cell; 1+: 1-3 signals/cell).

Results: PMT occurred in 11 males and 3 females (median age 53 y, range 35-73 y), all with clinically apparent TIO, as masses (mean $3.9 \mathrm{~cm}$, range $1.4-12 \mathrm{~cm}$ ) in bone $(\mathrm{N}=7$; femur (4), ilium, tibia, fibula) or soft tissues ( $\mathrm{N}=7$; thigh (3), buttock, ankle, arm, shoulder). All tumors showed typical features of PMT, as previously described. FGF23 mRNA was detected in 86\% (12/14) of PMT (3+: 6 cases, 2:5 cases, 1+: 1 case). One malignant PMT with metastasis had $3+$ staining in both locations. No nonPMT had detectable $F G F 23$ mRNA . Non-neoplastic osteocytes, which express low levels of FGF23 protein, did not show detectable staining. One patient with a FGF23 mRNA-negative PMT had normal serum FGF23 levels, consistent with production of an alternative phosphatonin.

Conclusions: Our novel semi-quantitative in situ hybridization assay for $F G F 23$ mRNA is a highly sensitive and specific adjunctive test for the diagnosis of PMT, applicable to formalin-fixed, paraffin-embedded tissues. In our experience, this method has increased specificity over FGF23 protein immunohistochemistry. Compared to RT-PCR, this method preserves tissue morphology, and reduces "false positives" related to detection of FGF23 mRNA expression in normal tissues.

43 Desmoid-Type Fibromatosis-Associated Gardner Fibromas: Prevalence and Impact on Local Recurrence

JM Cates, CM Coffin, TP Stricker. Vanderbilt University Medical Center, Nashville, TN. Background: Evaluation of surgical resection margins (SRM) fails to predict local recurrence of desmoid-type fibromatosis (DTF). Although Gardner fibroma (GF) is considered a precursor lesion of DTF, the prevalence of GF associated with DTF has not been systematically studied. We postulated that residual GF not excised during surgical resection of DTF may account for the limited prognostic value of SRM.

Design: Clinical and demographic data was abstracted from the medical records of 134 patients with DTF. Only 4 subjects had documented Gardner syndrome. Clinical and pathologic characteristics of GF-associated DTF and DTF without associated GF were compared using standard bivariate statistical methods. Log-rank tests of Kaplan-Meier recurrence-free survival (RFS) curves were also performed. Of 122 subjects followed after primary surgical resection, median follow-up was 2.3 years (range, 1 day-26.7 years) during which there were 44 local recurrences $(36 \%)$, with a median time to recurrence of 1.5 years (range, 4 months-16.2 years).

Results: Of 109 evaluable primary DTF resections, 34 (31\%) had an associated GF. In 8 additional cases, GF was present in either the incisional biopsy or local recurrence (39\% of all patients). In 23 of 30 (77\%) evaluable cases, GF was present at the surgical resection margin. GF was not associated with sex, age, adjuvant therapy or surgical resection margin status. DTF with GF were slightly larger than those without GF (mean, 7.4 vs. $5.8 \mathrm{~cm}, P=0.06$ ). DTF arising in the abdomen, abdominal wall or superficial trunk were associated significantly less often with GF than tumors of the extremities, head/neck region or deep trunk ( $22 \%$ vs. $54 \%, P<0.001)$. Median RFS for patients with DTF-associated GF was 4.8 years compared to 16.2 years in patients without associated GF. Primary resection at an outside facility $(P=0.004)$, deep, extra-abdominal location ( $P=0.008)$, and younger age at diagnosis $(P=0.004)$, were also associated with shorter RFS. RFS was not affected by surgical resection margin status or adjuvant therapy.

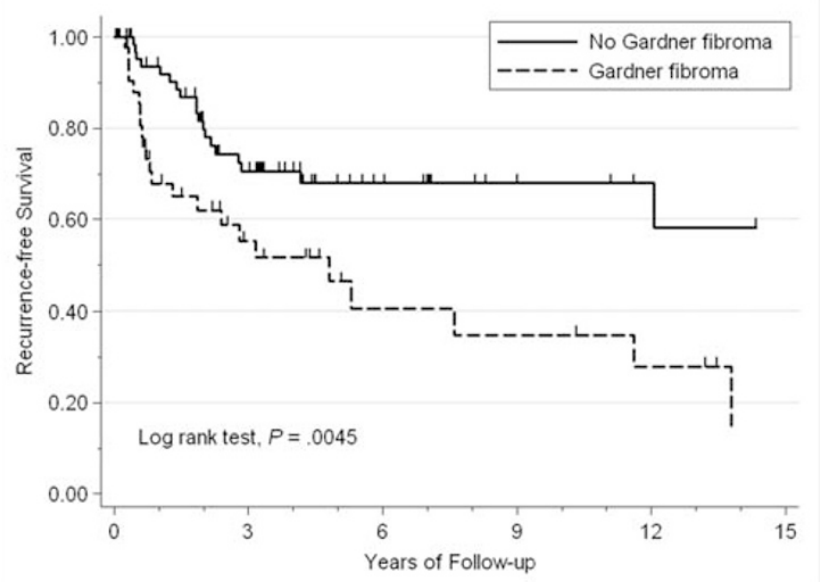

Conclusions: Gardner fibromas are associated with $39 \%$ of DTF. The presence of under-recognized residual DTF precursor lesions after surgical resection might account for the unpredictability of local recurrence in DTF.

44 Detailed Analysis of Surgical Resection Margins in DesmoidType Fibromatosis

JM Cates, CM Coffin, TP Stricker. Vanderbilt University Medical Center, Nashville, TN. Background: The prognostic value of surgical resection margins (SRM) in predicting recurrence of desmoid-type fibromatosis (DTF) is controversial. We analyzed whether the distance and tissue composition of negative margins, linear extent of positive margins, or adequacy of assessment affects the prognostic impact of SRM.

Design: The distance (clearance) and type of tissue composing the SRM was recorded for cases with negative SRM. The number of positive margins and linear extent of marginal involvement was recorded for cases with positive SRM. The number of tissue sections submitted for evaluation of SRM was also noted. Factors predictive of positive SRM were evaluated by logistic regression. Cox regression analysis of recurrence-free survival (RFS) was also performed.

Results: Of 128 surgical resection specimens, $71(56 \%)$ had positive SRM. For the 57 cases with negative SRM, the mean clearance was $0.22 \mathrm{~cm}$. Tissues composing the SRM were most often fibrous $(n=15)$, skeletal muscle $(n=15)$, or fibroadipose $(n=10)$. In cases with positive SRM, the mean linear extent of SRM involvement was $0.8 \mathrm{~cm}$. The number of tissue sections evaluating SRM was greater in cases with positive SRM (10.2 vs. $6.4, P<0.001)$. By logistic regression, only the number of tissue sections evaluating SRM was predictive of a positive SRM (odds ratio $1.15,95 \%$ CI 1.05-1.26). SRM status was not associated with age, sex, anatomic site, radiation therapy, size, or specimen type (primary resection vs. local recurrence). Patients with positive SRM received chemotherapy more often than patients with negative SRM. Of 122 subjects who underwent primary resection, only SRM clearance was predictive of local recurrence. Cox regression analysis of recurrence-free survival.

Cox regression analysis of recurrence-free survival.
\begin{tabular}{|l|l|l|}
\hline Margin status (R0 vs. R1/R2) & Hazard ratio & P \\
\hline R status (R0) & 0.80 & 0.58 \\
\hline R1 & 0.83 & 0.64 \\
\hline R2 & 0.69 & 0.63 \\
\hline Clearance (mm) & 0.03 & 0.02 \\
\hline Margin composition (fibrous tissue) & & 0.61 \\
\hline Adipose & 1.61 & 0.76 \\
\hline Fibroadipose & 1.29 & 0.72 \\
\hline Skeletal muscle & 1.35 & 0.90 \\
\hline Number of positive margins & 0.98 & 0.65 \\
\hline Linear extent of involvement & 1.20 & \\
\hline
\end{tabular}

Limiting Cox regression models to samples meeting increasingly stringent thresholds for predicted probability of positive SRM or by number of sections evaluating SRM had no effect on the hazard ratios for SRM status.

Conclusions: Resection margins fail to predict RFS for DTF. The number of positive SRM, linear extent of margin involvement and tissue composition also fail to predict RFS. However, decreasing clearance appears to be predictive of recurrence in cases with negative SRM. Although the optimum number of tissue sections for evaluation of SRM is unclear, most cases appear to be adequately sampled.

\section{5} Tumor

AL Cheah, SD Billings, JR Goldblum, P Carver, M Tanas, BP Rubin. Cleveland Clinic, Cleveland, $\mathrm{OH}$.

Background: Recurrent NAB2-STAT6 gene fusions have recently been identified in solitary fibrous tumors (SFT) by integrative sequencing. Nuclear expression of STAT6 by immunohistochemistry (IHC) discriminates meningeal SFT from its mimics in the central nervous system. Our aim was to examine the sensitivity and specificity of STAT6 IHC for SFT versus other morphologically similar soft tissue tumors.

Design: STAT6 expression was evaluated by IHC in 53 SFT of various sites and 99 soft tissue tumors in the histologic differential diagnosis. We used two antibodies (Epitomics and Santa Cruz) using FFPE whole sections and tissue microarray slides. Only nuclear staining of STAT 6 was considered positive. Staining was scored as: 0 (no staining), $1+$ $(1-25 \%), 2+(26-50 \%), 3+(>50 \%)$. Intensity was scored as weak, moderate or strong. 
Results: Our preliminary workup of the STAT6 Santa Cruz antibody showed excessive noise to signal ratio, which precluded accurate scoring. Thus, we elected to focus on the STAT6 Epitomics rabbit monoclonal antibody $(1: 100)$, which has not previously been examined by others, in our expanded study. Nuclear STAT6 staining was present in all SFT cases tested (53/53, sensitivity $100 \%)$. The majority of cases showed $3+$ and strong intensity staining. All tested cases of cellular angiofibroma (0/9), myofibroblastoma $(0 / 10)$, spindle cell lipoma $(0 / 10)$, benign fibrous histiocytoma $(0 / 13)$, dermatofibrosarcoma protuberans $(0 / 9)$, low-grade fibromyxoid sarcoma $(0 / 7)$, schwannoma (0/8), desmoid fibromatosis $(0 / 8)$, monophasic synovial sarcoma $(0 / 11)$, malignant peripheral nerve sheath tumor $(0 / 7)$, and mesenchymal chondrosarcoma $(0 / 7)$ were negative for STAT6 (specificity $100 \%$ ).

Conclusions: Our study provides further support that STAT6 is an extremely sensitive and specific marker for SFT and is a useful diagnostic adjunct in discriminating SFT from other soft tissue tumor mimics.

46 Expression of Growth-Associated Protein 43 (GAP43) in Malignant Peripheral Nerve Sheath Tumors Arising in the Neurofibromatosis-1 and Sporadic Settings

W-S Chen, P-L Chen, LP Dehner. Washington University in St. Louis, Saint Louis, MO; MD Anderson Cancer Center, Houston, TX

Background: The malignant peripheral nerve sheath tumor (MPNST) is a relatively uncommon type of soft tissue sarcoma arising from a peripheral nerve or extraneural soft tissues and showing nerve sheath differentiation. The diagnosis of MPNST can be one of the more challenging tasks due to its uncommon type, morphologic resemblance to other spindle cell neoplasms and lack of sensitive and specific immunohistochemical markers. The diagnosis is more straightforward in the clinical setting of neurofibromatosis-1 (NF-1), but problems are mainly centered on the sporadic (non-NF-1) MPNSTs. We recently reported the utility of a novel marker, Growth-associated protein 43 (GAP43), a membrane-associated phosphoprotein expressed in neuronal growth cones and Schwann cell precursors during neural development and axonal regeneration, in differentiating a set of benign and malignant nerve sheath tumors from other non-nerve sheath spindle cell neoplasms. We also showed that, in the setting of NF-1, GAP-43 has a sensitivity $(86 \%)$ superior to $\mathrm{S} 100$ protein $(62 \%)$. Here, we expanded our study to include a series of 19 sporadic (non-NF-1) MPNSTs.

Design: A series of 19 sporadic MPNSTs were selected from our surgical pathology archives, including 3 biopsies and 16 resection specimens. All cases showed at least positivity for one of the following three markers, namely S100 protein, collagen IV and Leu7. Immunohistochemical staining using anti-GAP43 and anti-S100 protein antibodies was performed on consecutive sections for each case.

Results: In this study, we showed that GAP43 is positive in $8 / 19$ cases $(42 \%)$, a result comparable to $\mathrm{S} 100$ protein $(\mathrm{n}=10 / 19 ; 52 \%)$. (Table 1.)

Table 1. S100 protein and GAP43 expression in sporadic MPNST

\begin{tabular}{|c|c|c|c|c|c|}
\hline & Neg & Pos & $0-5 \%$ & $25-50 \%$ & $>50 \%$ \\
\hline S100 & 9 & 10 & 5 & 3 & 2 \\
\hline GAP-43 & 10 & 9 & 6 & 2 & 1 \\
\hline
\end{tabular}

Conclusions: Even though GAP43 is a highly sensitive marker and superior to S100 protein for the diagnosis of MPNSTs in the NF-1 setting, the utility of this marker in the sporadic MPNSTs is more limited, and awareness of this caveat is important. Both comparative genomic hybridization studies and studies analyzing germline and somatic NF1 mutations suggest that the NF-1 and sporadic MPNSTs harbor different genetic changes, which may be reflected in part in the expression of GAP43. Our data provide further evidence that the MPNSTs arising from these two settings may reflect two different pathogenetic mechanisms.

\section{CCNB3 Immunohistochemistry Is Highly Specific for BCOR-} CCNB3 Fusion Sarcomas

S-J Cho, M Doral, A Sabnis, ML Loh, M Applebaum, SG Dubois, AE Horvai. UCSF, San Francisco, CA.

Background: Ewing sarcoma (ES) is a rare malignant small round blue cell tumor (SRBCT) characterized by chromosomal translocations resulting in fusions involving the EWSR I gene. SRBCT that morphologically and clinically fit a diagnosis of ES but lack $E W S R 1$ rearrangement pose a diagnostic dilemma. A subset of such ES-like sarcomas demonstrates fusion between the BCOR and CCNB3 genes (encoding the BCL6 corepressor and cyclin B3, respectively). Preliminary results suggest that these ES-like sarcomas may be detected by immunohistochemistry for CCNB3 protein. However, the diagnostic utility of CCNB3 immunohistochemistry in a large group of genetically characterized SRBCT has not been tested. We investigated the specificity of CCNB3 immunohistochemistry in SRBCT.

Design: We studied 48 SRBCT, previously characterized for EWSRI rearrangement. The SRBCT subtypes included conventional ES (classic clinicopathologic features of ES with EWSR I rearrangement, n=38), ES-like sarcoma (classic clinicopathologic features of ES but absent EWSRI rearrangement, $\mathrm{n}=3$ ), unclassified SRBCT (indeterminate $E W S R 1$ rearrangement status, $\mathrm{n}=5$ ) and other $E W S R 1$-associated SRBCT (desmoplastic small round cell tumor and clear cell sarcoma, $\mathrm{n}=2$ ). Formalin fixed-paraffin embedded tissue was stained using a combination of duplicate tissue microarrays, whole sections, or both using the CCNB3 polyclonal antibody HPA000496. Immunostaining for CCNB3 and detection of $B C O R-C C N B 3$ fusion by RTPCR was performed as previously reported (Pierron et al. Nat Genet. 2012;44:461-468). Strong cytoplasmic and/or nuclear staining was considered positive.

Results: Immunohistochemical staining results for CCNB3 are summarized in Table 1. Only one SRBCT $(1 / 48,2 \%)$ showed CCNB3 staining, which was cytoplasmic. RTPCR and DNA sequencing demonstrated a $B C O R$ exon 15 to $C C N B 3$ exon 5 fusion in this tumor.

Table 1. CCNB3 staining results in SRBCT.
\begin{tabular}{|l|l|l|l||}
\hline Diagnosis & $\mathbf{n}$ & CCNB3 positive & EWSR1 rearrangement \\
\hline Ewing sarcoma & 38 & $0(0 \%)$ & + \\
\hline Ewing-like sarcoma & 3 & $1(33 \%)$ & - \\
\hline Other EWSR1-associated SRBCT & 2 & $0(0 \%)$ & + \\
\hline Unclassified SRBCT & 5 & $0(0 \%)$ & Indeterminate \\
\hline Total & $\mathbf{4 8}$ & $\mathbf{1 ( 2 \% )}$ & \\
\hline
\end{tabular}

Conclusions: These results support that CCNB3 positivity by immunohistochemistry is infrequent, even among SRBCT that lack EWSR 1 rearrangement. Importantly, CCNB3 staining is highly specific since no EWSRI rearranged SRBCT demonstrated staining. Given the rarity of these tumors, it remains to be seen whether this antibody would be of value for routine clinical use. The oncogenic mechanisms of the BCOR-CCNB3 fusion protein may provide insight into the pathogenesis of this rare family of sarcomas.

\section{Reappraisal of Primary Spindle/Pleomorphic Sarcomas of Bone}

AP Dei Tos, A Righi, M Gambarotti, D Vanel, C Ferrari, S Benini, S Ferrari, P Picci. The Rizzoli Institute, Bologna, Italy; Department of Oncology, General Hospital of Treviso, Treviso, Italy.

Background: In contrast with the classification of soft tissue spindle/pleomorphic sarcomas, the classification of the much rarer spindle/pleomorphic sarcomas of bone is still limited to few entities as fibrosarcoma, undifferentiated pleomorphic sarcoma (formerly MFH), and leiomyosarcoma.

Design: 430 cases diagnosed as primary spindle/pleomorphic sarcomas of bone for the period 1937-2012 were retrieved from our archives. Thirty cases were excluded for the lack of material. Clinical records, pathology and radiology reports were reviewed. Secondary spindle/pleomorphic sarcomas of bone, cases without adequate slides, and spindle/pleomorphic sarcomas secondarily involving bone were excluded. Immunohistochemistry for cytokeratins AE1/AE3, SMA, desmin, S-100, CD34, MDM2, CD99, EMA, caldesmon, myogenin, TLE1, ALK, ERG, CD31, neurofilament, GFAP, vimentin and Bcl-2 was performed using commercial antibodies. EWSR1 and SYT gene rearrangements detected by FISH were performed when relevant. In all cases long term follow-up was available.

Results: On the basis of the histologic, clinico-radiological, immunohistochemistry and molecular review, of the 400 study cases, 326 were confirmed as primary spindle/ pleomorphic sarcomas of bone. Following reclassification fibrosarcomas dropped from 128 to 9 ; undifferentiated pleomorphic sarcoma from 141 to 47 ; malignant peripheral nerve sheath tumor from 10 to 8 and hemangiopericytoma from 10 to none. Leiomyosarcoma raised from 33 to 142; myoepithelioma from 2 to 12, and of synovial sarcoma from 1 to 25 . In addition other distinct entities were recognized as primary tumors of bone: myofibroblastic sarcoma (49 cases), solitary fibrous tumor ( 22 cases), mixofibrosarcoma ( 7 cases), pleomorphic rhabdomyiosarcoma (3 cases), pleomorphic liposarcoma (1 case) and pseudomiogenic hemangioendothelioma (1 case). Survival curves showed significant correlation with the revised subclassification.

Conclusions: Using strict diagnostic criteria and immunohistochemical and molecular analysis, commonly used diagnostic entities (fibrosarcoma, undifferentiated pleomorphic sarcoma/MFH and hemangiopericytoma ) tends to disappear whereas distinct entities tends to emerge. When primary spindle cell/pleomorphic sarcomas of bone are accurately sublassified, histoype appears as a significant predictor of outcome.

\section{STAT6 Immunohistochemistry Is Useful in the Diagnosis of Solitary} Fibrous Tumors

EG Demicco, PW Harms, RM Patel, SC Smith, SL Carskadon, S Camelo-Piragua, $J B$ McHugh, J Siddiqui, N Palanisamy, DR Lucas, AJ Lazar, W-L Wang. Mount Sinai Medical Center, New York, NY; University of Michigan Medical Center, Ann Arbor, MI; The University of Texas MD Anderson Cancer Center, Houston, TX

Background: Solitary fibrous tumors (SFT) are rare mesenchymal tumors that can be challenging to diagnose, especially in small biopsies, meningeal tumors and those tumors with anaplasia. Recently, SFTs have been discovered to harbor a recurrent gene fusion involving $N A B 2$ and STAT6, resulting in overexpression of STAT6. We assessed the diagnostic utility of nuclear expression of STAT6 in identifying SFT, and inquired if STAT6 might also play a role in other mesenchymal neoplasms.

Design: Tissue microarrays and tissue sections of a variety of mesenchymal tumors (see Table 1) were stained for STAT6 (1:200; M-20, SC-981, Santa Cruz), using an automated immunohistochemical stainer (Ventana). Nuclear and cytoplasmic staining were scored as 0 (absent-minimal blush), 1 (weak), 2 (moderate), or 3 (strong). Only $2-3+$ nuclear stain was considered positive.

Results: Of the 1419 cases assessed (Table 1), nuclear STAT6 was 2-3+ in 240 cases (17\%), including 202/250 SFT (81\%), 6/55 (11\%) unclassified sarcomas, 16/184 (9\%) desmoids, 4/63 (7\%) neurofibromata, and 3/154 (2\%) undifferentiated pleomorphic sarcomas. When present, STAT6 nuclear stain was seen diffusely in a majority of tumor cells. Amongst non-neoplastic tissues, scar was positive in 6/17 (35\%). Nearly $1 / 3$ of meningeal and intraabdominal tumors were negative for STAT6, compared to less than $10 \%$ of pleural tumors.

Conclusions: STAT6 immunohistochemistry is a relatively sensitive and specific test for SFT. Although occasionally moderate nuclear staining may be seen in other proliferations including unclassified sarcomas, neurofibromas, desmoids and scars, the role of STAT6 in these tumors is yet to be determined. Correlation with RT-PCR studies for NAB2-STAT6 fusion are ongoing. 
Table 1. STAT6 Immunohistochemistry in Soft Tissue Tumors

\begin{tabular}{|c|c|c|c|}
\hline Tumor Type & STAT6 positive & Total \# cases & \% Positive \\
\hline Alveolar soft part sarcoma & 0 & 25 & 0 \\
\hline Angiosarcoma & 0 & 67 & 0 \\
\hline Clear cell sarcoma & 1 & 19 & 5 \\
\hline $\begin{array}{l}\text { Dermatofibrosarcoma protuberans }+/- \\
\text { fibrosarcomatous transformation }\end{array}$ & 0 & 6 & 0 \\
\hline Desmoid & 16 & 184 & 9 \\
\hline Epithelioid sarcoma & 1 & 31 & 3 \\
\hline Fibrosarcoma & 0 & 3 & 0 \\
\hline Gastrointestinal stromal tumor & 1 & 26 & 4 \\
\hline Leiomyoma & 0 & 15 & 0 \\
\hline Leiomyosarcoma & 2 & 366 & 1 \\
\hline Liposarcoma & 1 & 16 & 6 \\
\hline Low grade fibromyxoid sarcoma & 0 & 5 & 0 \\
\hline Malignant peripheral nerve sheath tumor & 2 & 111 & 2 \\
\hline Neurofibroma & 4 & 59 & 7 \\
\hline Sinonasal glomangiopericytoma & 0 & 3 & 0 \\
\hline Solitary fibrous tumor & 202 & 250 & 81 \\
\hline Synovial sarcoma & 1 & 24 & 0 \\
\hline Unclassified sarcoma & 6 & 55 & 11 \\
\hline Undifferentiated pleomorphic sarcoma & 3 & 154 & 2 \\
\hline Total & 240 & 1419 & 17 \\
\hline
\end{tabular}

50 Perineurial Tumors with Typical and Atypical Features: A Clinicopathological Study of $\mathbf{4 6}$ Cases

$S$ Dotlic, JM Carter, AL Folpe. University Hospital Center, Zagreb, Croatia; Mayo Clinic, Rochester, MN.

Background: Perineuriomas are uncommon soft tissue tumors showing exclusively perineurial differentiation. Most perineurial tumors are histologically banal and behave in a benign fashion. However, rare perineurial tumors show atypical morphological features and behave aggressively; some of these tumors have been reported as perineurial malignant peripheral nerve sheath tumors. We studied a large series of perineurial tumors to better understand the natural history of these rare lesions, and better define criteria for malignancy in perineurial tumors.

Design: 46 cases previously coded as showing exclusively perineurial differentiation were retrieved. Cases were re-reviewed with attention to morphology (growth pattern, cellularity, mitotic rate, nuclear pleomorphism, and necrosis), and immunophenotype (EMA, GLUT1, claudin-1, CD34, and collagen IV). By definition, S100 protein expression was absent. Follow-up information was obtained.

Results: The tumors occurred in $19 \mathrm{M}$ and $27 \mathrm{~F}$ (median age 42 yrs.; range 12-78 yrs.) and ranged from $0.9-14 \mathrm{~cm}$ (median $3.5 \mathrm{~cm}$ ) in size. Common sites of occurrence included the extremities and trunk; the head/neck and viscera were rarely involved. Growth patterns, when evaluable, were circumscribed (15) and infiltrative (20). Nuclear grade was low (16), intermediate (18) and high (12). Cellularity was low (30), intermediate (11) and high (5). Necrosis was present in only 3 cases. Mitotic count was $0-100$ mitotic figures/ $50 \mathrm{HPF}$ (median 1/50 HPF). IHC results: EMA (46/46, only focal staining in 11/46), claudin-1 (30/43), GLUT-1 (35/42), CD34 (14/18), collagen IV (9/9). Clinical follow-up of $>10$ mos. was available for 10 patients (median 41 mos., range 10-216 mos.); 8 were alive without disease, 1 alive with disease and 1 dead of disease. Local recurrences occurred in 2 cases and metastases in 1 case. Local recurrences and metastases occurred only in tumors showing atypical features (e.g., high nuclear grade or cellularity, necrosis, mitotic count $>1 / 50 \mathrm{HPF}$ ).

Conclusions: A combination of careful morphological study and ancillary IHC should allow for the confident diagnosis of perineurial tumors, including those with atypical or worrisome morphological features. Preliminary follow-up data suggests that perineurial tumors lacking atypical morphological features behave in a clinically benign fashion, with adverse events occurring only in morphologically atypical tumors.

51 Genomic Analysis of $\mathbf{4 2 3}$ Sarcomas: A Single Institution Experience LA Doyle, VY Jo, JL Hornick, CP Raut, LM Sholl, NI Lindeman, CDM Fletcher, AJ Wagner. Brigham and Women's Hospital and Harvard Medical School, Dana Farber Cancer Institute, Boston, MA.

Background: Genomic analysis of tumors is becoming increasingly available, but the spectrum of abnormalities across large tumor sets is not well established. This study aimed to identify the frequency and types of genomic alterations detected in sarcomas using mutation hotspot-based analysis (OncoMap), and a more recent targeted exome sequencing platform (OncoPanel).

Design: FFPE tissue from 423 sarcomas was analyzed. OncoMap detects 472 mutations in 41 cancer-related genes by targeted multiplex PCR amplification and mass spectrometry analysis. OncoPanel surveys exonic DNA sequences of 275 cancerrelated genes and 91 introns across 30 genes for rearrangement by massively parallel sequencing using an Illumina HiSeq 2500 sequencer. MuTect and GATK were used to detect single nucleotide variants and indels, and VisCap to detect copy number variation. Results: OncoMap detected missense mutations in known cancer-related genes in 83 (21\%) of 398 samples. OncoPanel detected 158 missense mutations in 25 samples: of these 20 are known to be clinically significant or have known tumorigenic function and 138 were of unknown significance. As expected, OncoPanel had greater sensitivity in detection of missense mutations than allele-specific analysis; e.g., Oncomap detected 3 mutations in 27 dedifferentiated liposarcomas (DDLPS): Oncopanel detected 50 mutations in 7 DDLPS. Expected findings included KIT and PDGFRA mutations in GIST, CTNNBI mutations in desmoid fibromatosis and TP53 mutations in pleomorphic liposarcoma. $C T N N B 1$ mutations were also found in synovial sarcoma, MPNST and DDLPS. PI3K pathway (PIK3CA, AKT, MTOR) mutations were confirmed in myxoid liposarcoma and $R A S$ mutations in angiosarcoma. Promising findings included KRAS \& PIK3CA mutations in GIST, KRAS \& PTEN mutations in leiomyosarcoma, and PIK3CA \& TSC2 mutations in osteosarcoma. TP53 mutations were present in many different sarcoma types including some Ewing sarcomas. Mutations of uncertain significance involved the ARID family in various sarcoma types and DICER in leiomyosarcoma. High copy number gains were frequent in DDLPS. Low copy number gains and single gene deletions were frequent across various sarcoma types. Targeted validation of these findings in a larger panel of tumors is underway.

Conclusions: Given the wide spectrum of sarcoma types, a broad screening platform allows for greater detection of genomic events. Although the therapeutic and prognostic significance of the reported findings in most sarcoma types, other than GIST, remains to be determined, such data may help match clinical trials to the appropriate genotype.

$52 \quad$ STAT6 Is Amplified in a Subset of Dedifferentiated Liposarcoma

LA Doyle, DL Tao, A Marino-Enriquez. Brigham and Women's Hospital and Harvard Medical School, Boston.

Background: STAT6 is a member of the STAT family of cytoplasmic transcription factors. Recent studies have detected a recurrent intrachromosomal rearrangement in solitary fibrous tumor that results in the formation of a NAB2-STAT6 fusion oncogene. Nuclear expression of STAT6 by immunohistochemistry (IHC) is found in nearly all cases of SFT and serves as a useful diagnostic marker. STAT6 is located in 12q13, a chromosomal region containing well-characterized oncogenes that are commonly amplified in dedifferentiated liposarcoma (DDLPS), and expression of STAT6 has been reported to occur in a subset of DDLPS. The aim of this study was to determine the frequency of STAT6 expression and the underlying genetic mechanism in DDLPS. Design: STAT6 protein expression was evaluated in whole tissue sections from a wellcharacterized series of 35 DDLPS, all with nuclear MDM2 and CDK4 expression by IHC and/or cytogenetic features of DDLPS. IHC was performed following pressure cooker antigen retrieval ( $0.01 \mathrm{M}$ citrate buffer, $\mathrm{pH} 6.0)$ using a rabbit polyclonal STAT6 antibody (1:1000; sc-621; Santa Cruz Biotechnology, Santa Cruz, CA). Dual color FISH was performed with a commercial probe targeting the $12 \mathrm{q} 13$ region, which includes a SpectrumOrange probe spanning the STAT6 locus and a telomeric SpectrumGreen probe (Vysis, Abbott Molecular Inc, Des Plaines, IL), on 4- $\mu \mathrm{m}$ formalin-fixed paraffinembedded sections on all cases with STAT6 expression, and a subset of control cases without. 100 cells were scored per case. The presence of 5 or more orange signals was considered indicative of STAT6 amplification. Non-neoplastic diploid cells were used as internal controls in each case.

Results: $4 / 35$ cases (11\%) showed nuclear expression of STAT6 by IHC -3 with multifocal moderate to strong staining and 1 with weak focal staining- in either well-differentiated sclerosing or dedifferentiated components. FISH demonstrated amplification of STAT6 in the 4 positive cases; in contrast, FISH demonstrated no STAT6 amplification on 4 tumors negative for STAT6 expression by IHC. Of the 4 STAT6 amplified cases, 3 patients were male and 1 female, ranging in age from 51 to 76 years. Tumors were located in the mediastinum $(n=2)$, paratesticular soft tissue (1) and perirenal soft tissue (1). 3 patients received chemotherapy $+/$ - radiation therapy prior to resection.

Conclusions: STAT6 is amplified in a subset of DDLPS, which leads to nuclear expression of STAT6 that can be detected by IHC. These findings suggest a role for STAT6-mediated transcriptional activity in some cases of DDLPS, and highlight the genomic complexity and heterogeneity of DDLPS.

$53 \quad$ Nodular Fasciitis and Aneurysmal Bone Cyst Share the Novel SRSF3-USP6 Fusion Transcript: Further Evidence for Their Common Pathogenesis

MR Erickson-Johnson, B Evers, $C$ Roth, A Seys, $M$ Lonzo, $Y$ Asmann, $X$ Wang, MM Chou, AM Oliveira. Mayo Clinic, Rochester, MN; Children's Hospital of Philadelphia University of Pennsylvania, Philadelphia, PA

Background: Nodular fasciitis (NF) and aneurysmal bone cyst (ABC) are mesenchymal tumors that predominantly occur in younger patients and exhibit overlapping histologic features. While ABC is primarily a bone neoplasm, nodular fasciitis is exclusively encountered in the soft tissues. Recent studies have shown that both tumors harbor USP6 fusion genes but with distinct 5'-end partner genes. In this study we describe a novel fusion of the pre-mRNA splicing factor gene $S R S F 3$ (serine/arginine-rich splicing factor 3) to the ubiquitin protease USP6 in both NF and ABC

Design: One example each of $\mathrm{ABC}$ and NF were identified with the same $\mathrm{t}(6 ; 17)$ (p21;p13) by conventional cytogenetic analysis. Additional cases of NF $(n=7)$ and $\mathrm{ABC}(\mathrm{n}=6)$ that were previously shown to harbor USP6 rearrangement but without a known partner gene were also studied. Histologic diagnosis was confirmed in all cases according to the 2013 WHO classification. RNA was extracted from archival FFPE from both $\mathrm{ABC}$ and NF with $\mathrm{t}(6 ; 17)$. cDNA libraries were prepared using RNA was prepared for transcriptome analysis by paired-end sequencing using the Sure Select RNA target enrichment for Illumina Paired-End Multiplexed Sequencing kit (Agilent Technologies, Englewood CO). Transcriptome sequencing analysis was performed using a HiSeq 2000 sequencer (Ilumina Inc, San Diego, CA). SnowShoes-FTD algorithm for paired end mRNA-Seq data was used to identify any potential fusion gene (Asmann $\mathrm{Y}$ et al., 2011). Break-apart FISH using custom-designed probes for SRSF3 and USP6 and RT-PCR were used to confirm the rearrangement of these loci and presence of the novel fusion transcript, respectively.

Results: Transcriptome sequencing analysis identified the fusion of $S R S F 3$ exon 1 to USP6 exon 2 in an ABC with $\mathrm{t}(6 ; 17)$. RT-PCR confirmed this findings in both index $\mathrm{ABC}$ and $\mathrm{NF}$, and also identified a second splicing variant in which $S R S F 3$ exon 1 was fused to the USP6 exon 1. Among $6 \mathrm{ABC}$ and $7 \mathrm{NF}$ with previously unknown USP6 partner genes, FISH and RT-PCR identified 3 additional tumors ( $2 \mathrm{NF}$ and $1 \mathrm{ABC}$ ) with the same SRSF3-USP6 fusion. One additional ABC was identified with the typical NF fusion MYH9-USP6. 
Conclusions: We report the novel the fusion SRSF3-USP6 due to the translocation $\mathrm{t}(6 ; 17)$ in both NF and ABC, and the typical NF fusion MYH9-USP6 in a skeletal $\mathrm{ABC}$. These results support the impression that $\mathrm{ABC}$ and $\mathrm{NF}$ may belong to the same biologic spectrum of USP6-induced tumors with skeletal and soft tissue counterparts.

$54 \quad$ Frozen Section Analysis and Its Relation to Multiple Factors in the Decision to Pursue Total Joint Arthroplasty

DJ Fix, J Choi, AP Burke. University of Maryland Medical Center, Baltimore, MD. Background: The histologic evaluation has been considered to be the gold standard in diagnosis of periprosthetic joint infection. Numerous studies have examined the sensitivity and specificity of frozen section (FS) histologic analysis, with end comparison to tissue and hardware culture results. Our study is an attempt to review the utilization of FS in the surgical decision to pursue revision arthroplasty with reimplantation of hardware versus antibiotic therapy and postponed reimplantation, and to examine it in regards to concordance with other modalities which aid in this decision.

Design: A search (September, 2011 through July, 2013) for specimens from revision total joint arthroplasties was conducted. A total of 175 cases were identified. Data was collected for the following parameters: decision to pursue revision arthroplasty vs postponement, gross visualization, FS result, permanent section (PS) result, and operative culture results.

Results: Of 175 cases, 139 cases resulted in total joint athroplasty/revision (REV)and 36 with postponement of reimplantation (NOREV). In REV cases, $74 \%$ had a FS, with $92 \%$ being read as negative and $8 \%$ positive, as compared to FS performed in $42 \%$ of NOREV cases, of which $60 \%$ were positive and $40 \%$ negative, and a resultant positive predictive value (PPV) of $53 \%$ and a negative predictive value (NPV) of $94 \%$. This is contrasted with gross visualization, which was positive for infection in $67 \%$ and negative in $33 \%$ of NOREV cases, and positive in $4 \%$ and negative in $96 \%$ of REV cases, with a PPV of $80 \%$ and a NPV of $92 \%$. The NPV of FS compared to that for gross visualization indicates that it is a better test for "ruling out" infection. Average pair-wise Cohen's Kappa (CK) was 0.421 for all test categories, with notable CK values for operative decision versus gross visualization/FS/culture of $0.595 / 0.47 / 0.311$, respectively. FS results showed a moderate correlation with reimplantation $(\mathrm{CK}=0.47)$, less correlation with culture results $(\mathrm{CK}=0.355)$, producing both PPV and NPV are similar to that of previous studies. There was a good correlation between FS and PS $(\mathrm{CK}=0.667)$, with PS showing a small increase in sensitivity.

Conclusions: Gross visualization showed the highest correlation with decision regarding arthroplasty and culture results. FS is utilized more often in cases where the end decision is weighted towards REV rather than NOREV. The benefit of FS over gross inspection is not clearly documented by this study.

\section{5}

\section{Primary Myxoma of Bone}

J Forcucci, K Lindsey, C Welsh, D Lucas, MT Smith. Medical University of South Carolina, Charleston, SC; The University of Michigan Health System, Ann Arbor, MI. Background: Myxoma of bone is a rare, benign, but locally destructive, recurrent neoplasm. Infrequently, these lesions present in the craniofacial bones and mimic other soft tissue tumors, radiologically, clinically, and histopathologically. We present three pediatric cases of primary myxoma of bone emphasizing the clinicopathologic presentation and diagnostic evaluation of this rare entity.

Design: A retrospective search for cases of primary myxoma of bone within the surgical pathology files at two tertiary care centers was performed. Clinical, morphologic, and immunohistochemical data was analyzed.

Results: Three cases of primary myxoma of bone were identified. Each was located within the craniofacial bones of pediatric patients (age range 4-16). While the maxillary lesion was asymptomatic and discovered incidentally, the remaining cases found within the sphenoid were identified secondary to mass effect and visual disturbances. All patients underwent successful subtotal resection and had no evidence of distant disease or recurrence at follow up (range 0-4 years). Grossly, the specimens were composed of heterogenous gelatinous and fibrous soft tissue ranging in size from $4.8-5.3 \mathrm{~cm}$. The histologic sections show a sparsely cellular gray-blue myxoid stroma with evenly spaced bland stellate cells replacing medullary spaces between normal appearing bony trabeculae. In two cases, the stellate cells divide the myxoid material into vaguely polygonal shapes, while in the third case a lightly streaming pattern is present. A delicate capillary network, reminiscent of loose chicken-wire, is apparent in each case. The myxoid material is variable ranging from stringy, eosinophilic, and mucinous to more gray, finely granular, and amorphous. Within the maxillary lesion, an Alcian Blue stain illustrates the differential staining between epithelioid (glandular cells) and stromal mucins (myxoid material) with loss of blue staining following hyaluronidase digestion. Immunohistochemical stains elaborated pertinent negative staining of the stromal cells with S100, and SMA. The cells were positive for vimentin, and showed a low proliferation index with ki-67 staining.

Conclusions: Myxoma of bone is a rare lesion, particularly in the craniofacial bones, with a wide clinicopathologic differential diagnosis including metastatic atrial myxoma, chondroma, chordoma, and chondrosarcoma. Histologically, myxoma of bone recapitulates the features of other myxomas with bland stellate cells populating stromal material composed of mucins.
$56 \quad$ Clinicopathological and Immunohistochemical (IHC) Analysis of 46 Cases of Myxoinflammatory Fibroblastic Sarcoma (MIFS)

NM Harding-Jackson, JC Chang, E Zambrano, B Rubin, D Kazakov, C Bacchi, $H$ Dominguez-Malagon, J Lamovec, G Falconnieri, S Suster. Medical College of Wisconsin, Milwaukee, WI; Cleveland Clinic Foundation, Cleveland, OH; Charles University, Pilsen, Czech Republic; Consultoria em Patologia, Sao Paulo, Brazil; The National Cancer Institute of Mexico, Mexico City, Mexico; Institute of Oncology, Ljubljana, Slovenia; General Hospital S. Maria della Misericordia, Udine, Italy. Background: MIFS is a rare low-grade tumor of modified fibroblasts with a predilection for the distal extremities. Due to the diagnostic challenges posed by its varied morphology and nonspecific IHC features, further characterization may be helpful in distinguishing this entity from inflammatory processes and malignant lesions of higher metastatic potential.

Design: 46 cases of MIFS were retrieved. The clinical and histologic features were characterized, and immunophenotyping was performed using tissue microarray.

Results: Patient ages ranged from 20 to 89 (median 47), with no gender predilection. The anatomic distribution was as follows: foot $26 \%$, hand $24 \%$, forearm $17 \%$, calf $/ \mathrm{knee}$ $15 \%$, axial trunk $9 \%$, proximal arm $5 \%$, gluteus $2 \%$, and proximal leg $2 \%$. Histologic features showed varying amounts of myxoid and fibrotic stroma populated by a mixed inflammatory cell infiltrate. All 46 cases contained scattered large, bizarre-appearing tumor cells with vesicular chromatin, prominent inclusion-like nucleoli, and abundant eosinophilic and variably vacuolated cytoplasm. Seven of 46 cases $(15 \%)$ contained focal areas with a solid, syncytial proliferation of neoplastic cells that outnumbered inflammatory cells and spindle cells; however, areas of conventional MIFS were also present. By IHC, neoplastic cells exhibited strong nuclear positivity for cyclin D1 (86\%), cytoplasmic positivity with distinct perinuclear dot-like accentuation for CD10 (79\%), cytoplasmic positivity for PGP 9.5 (67\%), and membranous positivity for D2-40 with occasional perinuclear dot-like expression (67\%); tumor cells showed patchy positivity for CD63, CD34, and CD15 in 40\%, 35\%, and $29 \%$ of cases, respectively. Factor XIIIa and bcl-2 positivity were observed in $7 \%$ of cases. All cases were negative for AE1/AE3, S100, CD21, CD30, CD31, CD68, CD117, p63, ALK-1, FLI-1, GLUT-1, HBME-1, TLE-1, WT-1, MDM2, PAX-5, SOX-10, MITF, and beta-catenin.

Conclusions: The presence of focal syncytial neoplastic cell growth is reported. We also propose novel markers, which may be of diagnostic utility, including frequent expression of cyclin D1, CD10, PGP 9.5 and D2-40. Due to its high sensitivity and striking perinuclear dot-like pattern, CD10 and D2-40 may be of particular interest.

57 Spectrum of Primary and Secondary Mutations in Gastrointestinal Stromal Tumors: Molecular Analysis of 244 Cases

JF Hechtman, $M$ Hameed, A Oultache, D Kuk, P Chi, S Dogan, ME Arcila, M Ladanyi, K Nafa. Memorial Sloan-Kettering Cancer Center, New York, NY

Background: Primary and secondary mutation status of gastrointestinal stromal tumors (GISTs) influence treatment response and disease progression. Our objective was to characterize primary and secondary mutations in a large cohort of GISTs including recently described KIT exon 8 deletions.

Design: Primary site, size, and time from treatment start to sample acquisition of all GISTs that our lab performed mutation analysis on from 2009-2013 were reviewed. $K I T$ exons 9 and 11 were screened for indels by a sizing assay. KIT exons $9,11,13,14$, 17; and PDGFRA exons 12 and 18 were sequenced. To screen for KIT exon 8 indels in cases lacking the above KIT/PDGFRA mutations ("wild type" cases), we performed a sizing assay of this exon.

Results: Primary mutations were identified in 226 out of 244 (92.6\%) patients (table 1): 197 KIT mutations (exon 9: 1 colorectal, 21 small bowel; exon 11: 8 colorectal, 80 gastric; 75 small bowel, 2 omental, 2 esophageal; exon 13: 5 small bowel, 2 gastric, 1 multifocal familial), and 29 PDGFRA mutations (5 in exon 12: all gastric; and 24 in exon 18: 23 gastric, 1 omental). Primary mutation type was associated with site $(\mathrm{p}<0.001)$ but not size $(\mathrm{p}=0.4)$. All available wild type cases were negative for KIT exon 8 mutations $(0 / 13)$. Secondary mutations were identified in $33 / 68(48.5 \%)$ patients (table 1). Four of $32(12.5 \%)$ patients developed secondary mutations in KIT exons 13 and 17 between 12 and 15 months post imatinib. Secondary KIT mutations occurred mainly in the ATP binding pocket (exon 13: V643fs, Y646S, V654A, N655T; exon 14: T670I, N680K) and activation loop (exon 17: D820E/H/V, N822K/Y, Y823D). Five of 7 patients with primary KIT exon 9 mutations developed secondary KIT mutations: exon 11 (2), exon 13 (1) and exon 17 (2). Twenty-seven of 53 patients with KIT exon 11 mutations developed secondary mutations: exon 13 (11), exon 14 (1), exon 17 (14), and 1 KRAS exon 2 (G12V). One patient with a primary KIT exon 13 mutation (K642E) developed a second KIT exon 13 mutation (V654A). KIT primary mutation type correlated with secondary mutation $(\mathrm{p}=0.04)$. 


\begin{tabular}{|c|c|c|c|c|c|c|}
\hline \multicolumn{2}{|c|}{ Primary Mutations } & \multicolumn{5}{|c|}{ Secondary Mutations } \\
\hline Gene/Exon & Total & Exon 11 & Exons $13 / 14$ & Exon 17 & KRAS & Total \\
\hline KIT & 197 & 2 & 14 & 16 & 1 & 33 \\
\hline \multicolumn{7}{|l|}{ Exon $9(\mathrm{~N}=22)$} \\
\hline Ins & 19 & 2 & 1 & 2 & 0 & 5 \\
\hline Del & 2 & & & & & \\
\hline Missense & 1 & & & & & \\
\hline \multicolumn{7}{|c|}{ Exon $11(\mathrm{~N}=167)$} \\
\hline Ins & 11 & 0 & 0 & 1 & 0 & 1 \\
\hline Del & 111 & 0 & 9 & 13 & 1 & 23 \\
\hline Missense & 45 & 0 & 3 & 0 & 0 & 3 \\
\hline \multicolumn{7}{|c|}{ Exon $13(\mathrm{~N}=8)$} \\
\hline Missense & 8 & 0 & 1 & 0 & 0 & 1 \\
\hline PDGFRA & 29 & 0 & 0 & 0 & 0 & 0 \\
\hline \multicolumn{7}{|c|}{ Exon $12(\mathrm{~N}=5)$} \\
\hline Ins & 2 & & & & & \\
\hline Missense & 3 & & & & & \\
\hline \multicolumn{7}{|c|}{ Exon $18(N=24)$} \\
\hline Del & 6 & & & & & \\
\hline Missense & 18 & & & & & \\
\hline
\end{tabular}

Conclusions: Secondary KIT mutations are detectable between 12 and 15 months of imatinib initiation in $12.5 \%$ and in over $70 \%$ of patients after 33 months. KIT exon 8 indel mutations were not detected.

\section{Expression of ROS1 Predicts ROS1 Gene Rearrangement in} Inflammatory Myofibroblastic Tumors

JL Hornick, LM Sholl, CM Lovly. Brigham and Women's Hospital and Harvard Medical School, Boston, MA; Vanderbilt University School of Medicine and Vanderbilt-Ingram Cancer Center, Nashville, TN

Background: Inflammatory myofibroblastic tumor (IMT) is a distinctive, rarely metastasizing mesenchymal neoplasm composed of spindled or epithelioid cells with a prominent inflammatory infiltrate. Roughly $50 \%$ of IMTs harbor $A L K$ receptor tyrosine kinase gene rearrangements. Such tumors are usually positive for ALK by immunohistochemistry; the pattern of ALK staining correlates with the gene fusion partner. The molecular pathogenesis of ALK-negative IMTs is largely unknown. A recent study identified rearrangements of ROSI (which encodes a related receptor tyrosine kinase) in a subset of ALK-negative IMTs [J Clin Oncol. 31 (suppl), 2013 (abstr 10513)]. Immunohistochemistry for ROS1 has recently been shown to correlate with $R O S 1$ rearrangement in pulmonary adenocarcinoma. The purpose of this study was to determine whether immunohistochemistry for ROS1 could predict ROS1 rearrangement in IMT.

Design: In total, 30 IMTs were evaluated, including 21 ALK-positive tumors (10 confirmed to harbor $A L K$ rearrangements, with TPM3, CLTC, RANBP2, and FN1 fusion partners) and 9 ALK-negative tumors (including 2 known to harbor ROS1 rearrangements, with $Y W H A E$ and $T F G$ fusion partners). Immunohistochemistry was performed on whole tissue sections following pressure cooker antigen retrieval using a rabbit anti-ROS1 monoclonal antibody (clone D4D6; Cell Signaling Technology; 1:100 dilution). The results were scored as "positive" or "negative", and the pattern of staining was recorded.

Results: Three ALK-negative IMTs (including both tumors with known ROS1 rearrangements) showed immunoreactivity for ROS1, whereas all the ALK-positive IMTs were completely negative for ROS1. One ROS1-positive IMT (with ROS1$Y W H A E$ fusion) showed strong, diffuse cytoplasmic and nuclear staining; one case (with ROS1-TFG fusion) showed weak, granular cytoplasmic staining; and one case (genotype unknown) showed strong, diffuse cytoplasmic and dot-like staining. Next-generation sequencing to determine the ROSI fusion partner in the latter case is in progress.

Conclusions: Expression of ROS1 correlates with ROS1 rearrangement in IMT. These findings suggest that immunohistochemistry for ROS1 may be useful to confirm the diagnosis of a subset of IMTs, and also raise the possibility that some patients with clinically aggressive ALK-negative IMTs may be candidates for targeted therapy directed against ROS1. Whether the pattern of ROS1 staining correlates with the gene fusion partner remains to be determined.

\section{Delta-Like 1 Homolog (Dlk1) Is a Useful Marker for Diagnosis of}

\section{Rhabdomyosarcoma}

H Ikeda, H Kimura, S Kitamura, A Ooi. Kanazawa University Hospital, Kanazawa, Ishikawa, Japan.

Background: Delta-like homolog (Dlk1), a member of the epidermal growth factor family, is expressed in several organs during development, and has been detected in various carcinomas. Recently, the relationship between Dlk1 and skeletal muscle regeneration has been reported in rodent experiments and implicated to associate with myogenic tumor. Occasionally, it is difficult to discriminate between alveolar rhabdomyosarcoma and other small round cell tumor, also spindle or pleomorphic rhabdomyosarcoma and high grade sarcomas. We examined the expression of Dlk1 in rhabdomyosarcoma and various sarcomas, similar in morphology.

Design: We collected 40 cases of rhabdomyosarcoma ( 7 cases were embryonal type, 10 cases were alveolar type, 6 cases were spindle type, 17 cases were pleomorphic type). The cases of Ewing sarcoma, desmoplastic small round cell sarcoma were used for control disease of alveolar rhabdomyosarcoma, and the cases of leiomyosarcoma, liposarcoma, undifferentiated sarcoma were used for control disease of spindle/pleomorphic rhabdomyosarcoma. We examined Dlk1 expression using immunohistochemistry.
Results: The expression of Dlk1 was not seen in non-neoplastic skeletal muscle in human. The cytoplasmic staining of Dlk1 was found in rhabdomyosarcoma cells in various degree. The percentage of Dlk-1-positive rhabdomyosarcoma was $100 \%$ in embryonal type, $100 \%$ in alveolar type, $83 \%$ in spindle type, and $88 \%$ in pleomorphic type. Dlk-1 was almost negative in control diseases except 1 case of Ewing sarcoma and undifferentiated sarcoma. The distribution of DLK-1-positive cells were not similar to myogenin or desmin.

Conclusions: The expression of Dlk-1 was frequently seen in all subtype of rhabdomyosarcoma and Dlk-1 was almost negative in sarcomas similar in morphology. Dlk-1 seems to be useful marker for the histological diagnosis of rhabdomyosarcoma and also decision of tumor area.

60 Lentiviral Delivered PPARg ShRNA Improves Bone Microarchitecture by Altering the Balance of Osteogenesis and Adipogenesis

AW James, J Shen, K Khadarian, S Pang, C Chung, R Goyal, G Asatrian, X Zhang, $K$ Ting, C Soo. UCLA, Los Angeles, CA.

Background: Skeletal aging is associated with an increase in bone marrow adiposity. Recent study has focused on the role of adipokines and their negative impact on bone maintenance. A novel therapy for osteoporotic bone loss may be to target intramedullary adipogenesis. PPARgamma (PPARg) is considered the master regulator of adipogenesis. Here, we designed a lentiviral delivery system for PPARg shRNA, and examined its effects in an intramedullary injection model.

Design: PPARg shRNA was delivered by a lentiviral vector. In vitro testing confirmed purity, concentration, and efficacy. Next, control (GFP) lentivirus or PPARg shRNA expressing lentivirus were delivered by injection into the femoral intramedullary space of SCID mice. At four weeks post injection, analysis was performed, including high resolution micro CT, histology and histomorphometry, and immunohistochemistry for markers of adipocytes, OBs, and OCs.

Results: PPARg shRNA delivery reduced Pparg expression in BMSC, accompanied by a reduction in adipogenic differentiation.

A
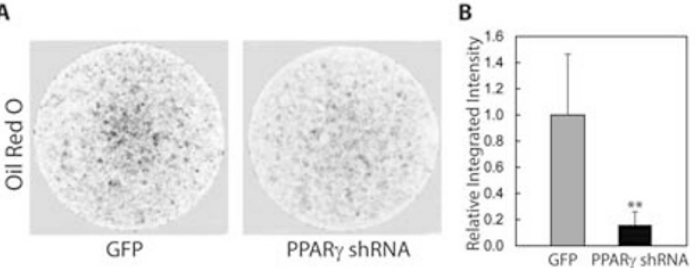

C

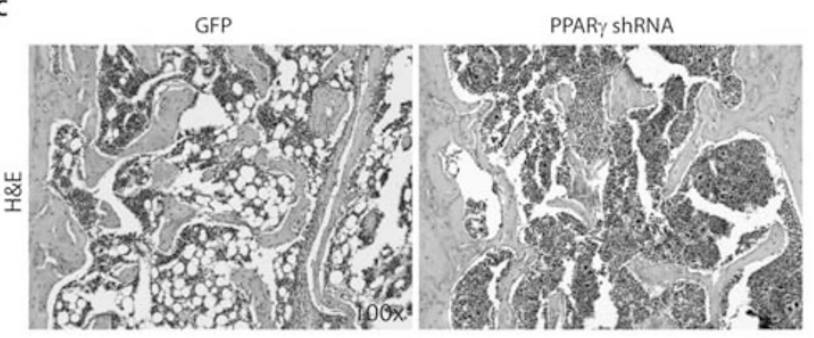

Figure 1

In vivo, a near total reduction in mature marrow adipocytes was observed. This was accompanied by reductions in adipocyte markers PPARg and FABP4. 


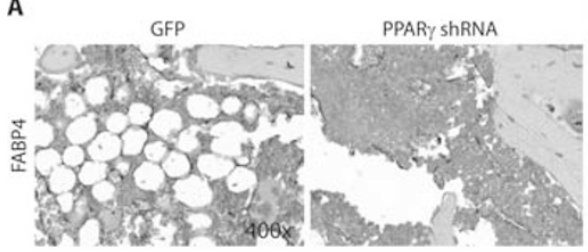

C

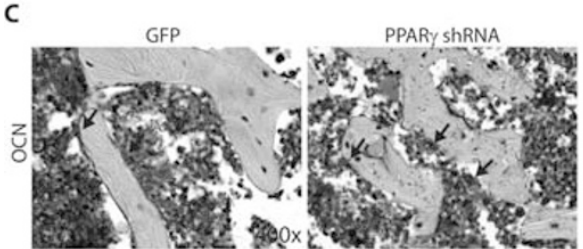

E

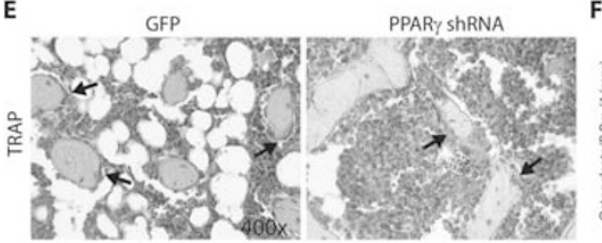

D
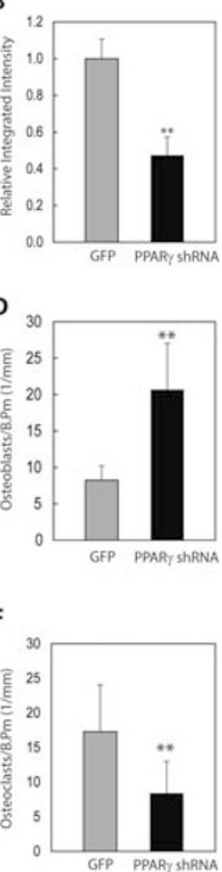

Figure 2

Unexpectedly, trabecular bone was increased by microCT and histomorphometric analyses. Immunohistochemistry demonstrated an increase in the number of bone-lining OBs and a decrease in multinucleated OCs.

Conclusions: These findings suggest that loss of PPARg in the bone marrow compartment has a significant role beyond anti-adipogenic effects. Specifically, we found improved trabecular bone microarchitecture, increased osteoblastic and decreased osteoclastic effects. PPARg silencing may either have had a direct effect on OB and OC cell types, or altered the adipokine milieu to favor intramedullary bone formation.

61 Epithelioid Malignant Peripheral Nerve Sheath Tumor: Clinicopathologic Features of 48 Cases

VY Jo, CDM Fletcher. Brigham and Women's Hospital and Harvard Medical School, Boston, MA.

Background: Epithelioid malignant peripheral nerve sheath tumor (EMPNST) is rare and may be confused with melanoma. It differs from conventional MPNST by showing diffuse S-100 staining, infrequent association with NF1, and occasional origin in a schwannoma. Loss of INI1 expression is seen in a subset of tumors. We describe the clinicopathologic features of this distinct variant in a large series.

Design: 48 cases were identified in consult files (2000-2013). H\&E and immunohistochemical stains were examined. Follow-up data was obtained from referring pathologists.

Results: Patients were 23 men and 25 women, median age 42.5 years (range 6-80). Anatomic sites were: lower extremity (20), upper extremity (3), neck (2), trunk (16), lip (2), visceral (5). In somatic sites, 4 were dermal, 24 subcutaneous, and 7 subfascial. Grossly tumors were well-circumscribed and cystic, firm, or lobular; 4 were associated with a nerve. Size range was $0.4-16 \mathrm{~cm}$ (median 2.95). Most tumors showed characteristically multilobulated growth of relatively uniform but atypical epithelioid cells; 4 were circumscribed nodules and 2 had solid sheetlike growth. Tumor cells had round nuclei and abundant amphophilic cytoplasm; some had spindled or myxoid foci. 7 tumors showed mild atypia with vesicular round nuclei and small nucleoli. The remainder showed moderate-to-severe atypia with irregular vesicular nuclei and prominent nucleoli. Mitoses ranged from 0-46/10 HPF; atypical forms were seen in 7 cases. 6 tumors had necrosis, all with at least moderate atypia and frequent mitoses. 3 tumors arose in a schwannoma and 1 in a neurofibroma (in an NF1-patient). All tumors expressed S-100: 46 strong and diffuse, 2 multifocal. Mart-1/Melan A(0/45) and HMB$45(0 / 47)$ were negative. GFAP was often positive (23/38); keratin was negative $(0 / 23)$. For EMA, 4/25 showed rare positive cells and a capsule was highlighted in 6 cases. INI1 expression was lost in 28/42 tumors $(67 \%)$. Most patients were treated by resection (5 marginal, 15 with wide margins). Follow-up data is available for 14 patients so far (range 3-144 months): 3 developed local recurrence, 3 had metastases, 6 are alive with no evidence of disease; 2 died of disease.

Conclusions: EMPNST is a distinct variant that affects adults most frequently on the lower extremity, though a wide age range and site distribution are seen. Most are diffusely S-100 positive and two thirds of tumors show INI1 loss. Based on preliminary data, there is a risk of recurrence and metastases; better prognosis may be associated with completely excised small superficial tumors.
62 Relevance of FNCLCC Grade and MDM2 Amplification Levels in Dedifferentiated Liposarcoma

G Jour, A Gullett, M Liu, BL Hoch. University of Washington Medical Center, Seattle, WA; University of Washington, Seattle, WA.

Background: Dedifferentiated Liposarcoma (DDLS) is a form of liposarcoma occurring mostly in the retroperitoneum. A limited number of studies have shown no reliable prognostic factors other than anatomical site. Molecular markers of tumor behavior, including amplification levels of $M D M 2$ and $C D K 4$ genes, have been explored in only a very small number of cases. Furthermore, the prognostic value of grading is uncertain in DDLS. Herein, we investigate whether FNCLCC grade and MDM2 gene amplification levels have prognostic value in DDLS in terms of local recurrence (LR) and disease free survival (DFS).

Design: 50 cases were retrieved. Cases were reviewed and FNCLCC grade was scored for the dedifferentiated component. Testing for $M D M 2$ gene amplification was performed by fluorescence in situ hybridization (FISH). 40 nuclei were counted for each case. Amplification was categorized as high-level when one nucleus had 20 or more MDM2 copies, and as low-level when it had less than $<20$ copies. Follow up data was obtained through chart review and phone calls to clinicians. Log rank test and Cox proportional hazards models were used to determine the effect of grade and level of amplification on LR and DFS outcomes.

Results: Our series includes 50 patients [male $n=28$, female $n=22$ ] with an average age of 63 years old (range 28-88) and a median follow up of 28 months (range 2-120). Tumors were graded as grade $1(6 \%)$, grade $2(58 \%)$, and grade $3(36 \%)$. Tumor sites included retroperitneum $(68 \%)$ and other sites $(32 \%)$. When adjusted for age, sex, site, tumor size, and margin status, grade 3 patients had a higher recurrence rate than grade 1 and $2(\mathrm{HR}=2.07,95 \% \mathrm{CI}: 1.24,7.62 ; \mathrm{p}=0.015)$. Patients with high level of MDM2 amplification $(>20)$ had higher recurrence rate on univariate $(\mathrm{p}=0.028)$ but not on multivariate analysis $(\mathrm{HR}=1.69,95 \% \mathrm{CI}: 0.73,3.94 ; \mathrm{p}=0.221)$. No significant association was seen between FNCLCC grade or level of amplification and DFS. Furthermore, no significant association was noted between amplification and grade $(\chi 2=1.48, \mathrm{p}=0.221)$.

Conclusions: FNCLCC grade 3 dedifferentiation confers a worse prognosis in DDLS in terms of local recurrence. $M D M 2$ amplification level remains a useful diagnostic tool in DDLS, but has no prognostic value in terms of local recurrence. Additional studies investigating potential biomarkers such as $C D K 4$ gene are warranted. This may yield an integrative grading system for DDLS combining both molecular and morphological parameters.

63 Loss of p16 (CDKN2A) Expression Is Rare in Dedifferentiated Liposarcoma and Does Not Promote Tumor Progression

$Y$ Kang, AE Horvai. UCSF, San Francisco, CA.

Background: Well-differentiated liposarcoma (WDL) and dedifferentiated liposarcoma (DDL) constitute the most common subgroup of liposarcomas and may represent a time-dependent form of tumor progression from lipogenic to non-lipogenic phenotype. WDL and DDL share amplification of chromosome subregion 12q13-15 resulting in amplification of the $M D M 2$ and $C D K 4$ genes. Overexpression of the MDM2 and CDK4 proteins likely leads to loss of cell cycle G1 control and initiation of tumorigenesis but the mechanisms of progression from WDL to DDL are poorly understood. Recent studies indicate that the p16 protein (a product of the $C D K N 2 A$ gene) is commonly expressed in WDL. The p16 tumor suppressor plays an antagonistic role to CDK4 by preventing CDK4-mediated inactivation of $\mathrm{Rb}$ and loss of $\mathrm{p} 16$ occurs during neoplastic progression in some malignancies. In a subset of liposarcomas, loss of p16 may compensate for absence of $C D K 4$ amplification, but the role of p16 in progression is unknown. In this study, we investigate the correlation between $\mathrm{p} 16$ expression and progression to a nonlipogenic phenotype, and the relationships between p16 expression and copy number changes of $C D K N 2 A$ and $C D K 4$.

Design: Twenty-one cases of DDL with chromosome 12q13-15 amplification previously confirmed by array comparative genomic hybridization (aCGH) and known MDM2 and CDK4 expression status were identified from the departmental archives. Matched lipogenic and non-lipogenic components of the DDL were immunostained for p16 using standard methods. Staining was scored on a scale of $0-3+$, based on the fraction of cells and intensity of staining. The p16 results were compared to aCGH and IHC data for copy number changes and CDK4 expression, respectively.

Results: All DDL were positive for p16 with complete concordance between staining scores for matched lipogenic and non-lipogenic components. Specifically, 20 tumors $(95 \%)$ demonstrated strong, diffuse $(3+)$ p 16 staining in both components. One tumor $(5 \%)$ showed weak, focal $(1+)$ p16 staining in both components and a corresponding decreased copy number of $9 \mathrm{p} 21.3$, in the region of $C D K N 2 A$. This tumor also lacked $C D K 4$ gene amplification and CDK4 protein expression in both components.

Conclusions: The concordance between p16 staining in matched components of DDL suggests that p16 loss likely does not mediate progression to a non-lipogenic phenotype. In a small subset of DDL, allelic deletion of $C D K N 2 A$ results in decreased p16 expression, and may compensate for absent $C D K 4$ amplification, but this is likely an early event in tumorigenesis.

64 Diagnostic Utility of p16 Immunohistochemistry in Distinguishing Dedifferentiated Liposarcomas from Other Retroperitoneal Sarcomas $Y$ Kang, AE Horvai. UCSF, San Francisco, CA.

Background: The family of well-differentiated and dedifferentiated liposarcomas (WDL and DDL, respectively) are among to most common soft tissue sarcomas in adults. Both WDL and DDL demonstrate amplification of chromosome subregion $12 \mathrm{q} 13-\mathrm{q} 15$ with resultant overexpression of the $M D M 2$ and $C D K 4$ genes. Consequently, immunohistochemistry (IHC) for the MDM2 and CDK4 proteins is often used to 
confirm the diagnosis of WDL and DDL. Recent studies have suggested that IHC for p16 (the product of the $C D K N 2 A$ gene) may be a useful adjunct to MDM2 and CDK4 to differentiate WDL from benign adipocytic tumors. However, the utility of p16 IHC to distinguish DDL from morphologic mimics is unknown. In this study, we examine the utility of p16 IHC in differentiating DDL from other retroperitoneal spindle cell malignancies.

Design: This study compared 44 high-grade DDL to 32 other high-grade retroperitoneal sarcomas for the presence of p16 immunohistochemistry. All DDL showed 12q13-15 amplification and/or positive IHC for MDM2 and CDK4, whereas the control sarcomas lacked MDM2, CDK4 and a WDL component. Based on clinicopathologic features, the control sarcomas consisted of leiomyosarcoma (LMS, $n=10$ ), undifferentiated pleomorphic sarcoma (UPS, $n=13$ ), sarcomatoid carcinoma ( $\mathrm{SCA}, \mathrm{n}=7$ ), endometrial stromal sarcoma (ESS, $\mathrm{n}=1$ ) and malignant gastrointestinal stromal tumor (GIST, $\mathrm{n}=1$ ). IHC for $\mathrm{p} 16$ was performed on paraffin-embedded whole tissue sections using standard methods and the slides were scored 0 to $3+$ based on intensity and fraction of cells staining.

Results: IHC stain results for $\mathrm{p} 16$ are summarized in Table 1. Although p16 staining was more common in DDL than the control sarcomas as a group, the difference was greatest when compared to SCA. By contrast, retroperitoneal LMS could not be distinguished from DDL by p 16 status. The sensitivity of $\mathrm{p} 16$ for DDL was $98 \%$ with negative predictive value of $91 \%$, but specificity $(31 \%)$ and positive predictive value $(67 \%)$ were low when comparing to other retroperitoneal sarcomas.

Table 1. p16 immunohistochemical expression in retroperitoneal sarcomas.

\begin{tabular}{|c|c|c|c|c|c|c|}
\hline Diagnosis & $n$ & p16 positive & & ng sc & & \\
\hline & & & 0 & $1+$ & $2+$ & $3+$ \\
\hline DDL & 444 & $43(98 \%)$ & & 11 & & $\sqrt{42}$ \\
\hline Other sarcomas & $\sqrt{32}$ & $22(69 \%)$ & {$[10$} & 1 & $\sqrt{3}$ & |18 \\
\hline LMS & 10 & $10(100 \%)$ & & & 2 & 8 \\
\hline UPS & 13 & $9(69 \%)$ & 4 & 1 & 11 & 7 \\
\hline SCA & $\sqrt{7}$ & $2(29 \%)$ & 5 & & & 2 \\
\hline ESS & $\sqrt{1}$ & {$[0(0 \%)$} & 1 & & & \\
\hline Malignant GIST & 1 & $1(100 \%)$ & & & & $\sqrt{1}$ \\
\hline
\end{tabular}

Conclusions: These results demonstrate the utility of p16 in the diagnosis of retroperitoneal sarcomas insofar as a negative p 16 result largely excludes the diagnosis of DDL. However, a positive result lacks sufficient specificity to exclude other sarcomas, especially LMS.

\section{Impact of Fluorescence In Situ Hybridization Analysis on the Final} Diagnosis of Dermatofibrosarcoma Protuberans: About $\mathbf{4 4 8}$ Cases

M Karanian, JM Coindre, G Perot, F Chibon, A Neuville. Institut Bergonie, Bordeaux, France.

Background: Dermatofibrosarcoma protuberans (DFSP) is a rare superficial mesenchymal tumor of intermediate malignancy, locally aggressive but rarely metastasizing. Cytogenetically, most DFSP is characterized by chromosomal rearrangements resulting to the COL1A1-PDGFB fusion gene. This abnormality can be detected by fluorescence in situ hybridization (FISH) analysis in routine practice. The aim of this study was to evaluate the role of the molecular analysis in the diagnosis of DFSP.

Design: 448 cases referred for second opinion or for confirmation of a diagnosis of DFSP since 2007 to 2013 were studied. For these cases a FISH had been performed to detect the COL1A1-PDGFB fusion gene. All cases were reviewed by two pathologists and classified in three categories according to the probability of the DFSP diagnosis before molecular analyses. The case was "certain" when DFSP was the only possible diagnosis; "probable" when DFSP remained the first diagnosis, but other differential diagnosis existed; "possible" when DFSP was considered as a differential diagnosis. Final diagnosis was supported by clinico-pathological findings and results of FISH analyses. Immunohistochemical analysis of CD34 was systematically performed and other markers when necessary. FISH assay was performed using the Histology FISH accessory kit $\left(\right.$ Dako $\left.^{R}\right)$ and home-made probes.

Results: For the 200 "certain" cases, 178 (89\%) FISH analyses were positive, 7 (3.5\%) negative and $15(7.5 \%)$ non interpretable (NI). For the 121 "probable" cases: $103(85 \%)$ FISH analyses were positive, $8(6 \%)$ were NI and $10(9 \%)$ were negative. For these 10 cases, final diagnoses were: 1 low-grade myofibroblastic sarcoma, 3 undifferentiated sarcomas, 2 solitary fibrous tumors, 1 spindle cell lipoma, 1 perineurioma, 2 undifferentiated spindle cell tumors without malignant evidence. For the 127 "possible" cases: 91 (71\%) FISH analyses were negative, $14(12 \%)$ were NI and $22(17 \%)$ were positive. In these 22 cases, 7 undifferentiated sarcomas were finally considered as fibrosarcomatous DFSP, 4 myxofibrosarcomas as myxoid DFSP, 2 dermatofibromas, 2 reactive lesions, 1 solitary fibrous tumor, 1 perineurioma, 1 myoepithelioma, 1 benign nerve sheath tumor, and 3 undifferentiated spindle cell tumor without malignant evidence as DFSP. FISH analysis has been helpful for confirming the diagnosis of DFSP in $23 \%$ of cases $(103 / 448)$ and necessary for the diagnosis of DFSP in $5 \%$ of cases $(22 / 448)$. Conclusions: This study highlights the important role of the FISH analysis in the diagnosis of DFSP.

\section{FOSL1 Protein Is Differentially Overexpressed in Desmoplastic} Fibroblastoma and a Subset of Fibroma of Tendon Sheath

I Kato, T Motoi, Y Fukuda, A Yoshida, M Ogawa, H Koiwai, S Horiguchi, T Goto, $T$ Hishima. Tokyo Metropolitan Cancer and Infectious Diseases Center Komagome Hospital, Tokyo, Japan; National Cancer Center Hospital, Tokyo, Japan.

Background: Desmoplastic fibroblastomas (DFBs) and fibromas of tendon sheath (FTSs) are benign fibroblastic tumors that comprise bland spindle cells. A recurrent chromosomal rearrangement involving 11q12 locus has been reported in DFBs and FTSs, suggesting a genetic link between the 2 entities. Recently, a microarray analysis of DFBs revealed mRNA elevation of FOSL1, which encodes a nuclear leucine zipper protein. As the FOSL1 gene is located close to 11q12, it has been proposed as a candidate involving the 11q12 rearrangement. We here explored the diagnostic potential of FOSL1 immunohistochemistry by studying DFBs, FTSs and their histological mimics. In addition, we investigated FOSL1 rearrangement status in selected cases.

Design: FFPE samples of 9DFBs and 10FTSs were immunostained by using antiFOSL1 monoclonal antibody. Ten desmoid-type fibromatoses (DMFs), 10 nodular fasciitis (NDs), 10 superficial fibromatoses (SFs), 6 spindle cell lipomas (SLs), and 3 neurofibromas (NFs) were also stained as histological mimics. Only nuclear reactivity was considered significant. The results were graded as $2+(>50 \%$ of tumor cells stained with strong intensity), $1+(10-50 \%$ of cells stained with strong intensity or $\geq 10 \%$ of cells stained with weak intensity), or 0 ( $<10 \%$ of cells stained with weak intensity). FOSL1 gene status was examined on FFPE samples of 6DFBs, 2FTSs, 1ND, and 1DMF, by using dual-color chromogenic in situ hybridization (CISH) with custom-made breakapart probes spanning FOSL1.

Results: All DFBs (9/9) showed 2+ FOSL1 immunopositivity, whereas 50\% (5/10) of FTSs showed $2+$ reactivity. The remaining FTSs showed $1+(30 \%, 3 / 10)$ or $0(20 \%$, $2 / 10$ ) staining. Histological appearance of FTSs did not predict FOSL1 expression status. Other spindle cell tumors showed $1+(25 \%, 10 / 40)$ or $0(75 \%, 30 / 40)$ reactivity. None of the cases examined by CISH showed FOSL1 break-apart signals.

Conclusions: Strong diffuse immunoreactivity of FOSL1 distinguishes DFBs from histological mimics such as DMFs. In contrast, similar overexpression was seen only in a subset of FTSs, suggesting that FTSs may not be a uniform tumor entity; FOSL1overexpressing subset may be related to DFBs and might harbor 11q12 rearrangement. Our CISH analysis indicated that the FOSLl is not rearranged in DFBs or FTSs, and suggested that FOSL1 expression may be induced by other mechanisms.

\section{MYC Immunohistochemistry and FISH Are Useful in the Diagnosis} of Postradiation Angiosarcoma

V Khachaturov, R Kwan, J Tripodi, RA Garcia, V Najfeld, EG Demicco. Mount Sinai Medical Center, New York, NY.

Background: Postradiation vascular neoplasia in the breast includes benign atypical vascular lesions (AVL) and malignant angiosarcomas (AS). Accurate differentiation between the two lesions is essential for appropriate clinical management and prognosis. Recently, recurrent genetic abnormalities, including high level amplification of $M Y C$, FLT4 and 10p12.33, were reported in postradiation AS and not AVL or primary AS. In order to determine the diagnostic value of these molecular alterations and assess immunohistochemical correlates, we evaluated a series of postradiation vascular proliferations of the breast for amplification of MYC, FLT4, 10p12.33 and Prox 1 and for MYC expression by immunohistochemisty (IHC).

Design: Postradiation vascular lesions of the breast were retrieved from institutional pathology archives. H\&E slides were reevaluated by 2 soft tissue pathologists and classified as AVL or AS. Formalin-fixed paraffin-embedded (FFPE) tissue was stained for MYC (Y69, Ventana). Nuclear stain was scored from 1+ (weak) to 3+ (strong), with only $2-3+$ staining considered positive. Fluorescence in situ hybridization (FISH) was performed on 4-mm FFPE sections using locus specific custom probes for MYC (8q24.21), FLT4 (5q35.3), 10p12.33 and Proxl (1q41) (Empire Genomics). Amplification was defined as $\geq 9$ signals in $\geq 25 \%$ of counted nuclei.

Results: Patients included 18 females with $22 \mathrm{AS}$ and 8 with AVL. MYC and FLT4 amplification were detected in $86 \%$ and $9 \%$ of AS, respectively. No amplification of Prox 1 or 10p12.33 was detected in any AS. All AVLs tested showed no amplification for MYC, FLT4, 10p12.33 and Proxl (Table 1). All MYC IHC positive cases demonstrated $M Y C$ amplification, while 5 cases with $M Y C$ amplification gave false negative IHC results. All cases of AVL were negative for MYC (Table 2).

Table 1. Amplification of Targeted Loci by FISH

\begin{tabular}{|c|c|c|c|c|}
\hline & MYC & FLT4 & Prox1 & $10 \mathrm{p} 12.33$ \\
\hline $\mathrm{AS}(\mathrm{n}=22)$ & $19(86 \%)$ & $2(9 \%)^{*}$ & 0 & 0 \\
\hline $\operatorname{AVL}(\mathrm{n}=8)$ & 0 & 0 & 0 & 0 \\
\hline
\end{tabular}

* one case showed coamplification of MYC

\begin{tabular}{|c|c|c|}
\hline & $\widehat{A S}$ & $A V L$ \\
\hline MYC IHC & MYC AMP FISH & MYC AMP FISH \\
\hline Negative & $1 / 4(25 \%)$ & $0 / 7$ \\
\hline $1+$ & $5 / 5(100 \%)$ & $0 / 1$ \\
\hline $2+, 3+$ & $13 / 13(100 \%)$ & None \\
\hline Total & $19 / 22(86 \%)$ & $0 / 8$ \\
\hline
\end{tabular}

Conclusions: Our results support that $M Y C$ amplification is a reliable test to distinguish postradiation AS from AVL, particularly in small biopsies or cases with overlapping histologic features. MYC IHC is a useful screen for MYC amplification, but may lead to false negative results. Suspected AS with weak staining should be tested for MYC amplification by FISH for definitive confirmation.

68 A Recurrent Point Mutation in MYOD1 Defines a Clinically Aggressive Subset of Embryonal Rhabdomyosarcoma

$S$ Kohsaka, N Shukla, N Ameur, D Lim, A Viale, N Socci, L-X Qin, R Sciot, J Bridge, $S$ Singer, L Wexler, FG Barr, S Dogan, JA Fletcher, M Ladanyi. Memorial Sloan-Kettering Cancer Center, New York, NY; University Hospital Gasthuisberg, Leuven, Belgium; University of Nebraska Medical Center, Omaha, NE; National Cancer Institute, Bethesda, MD; Brigham and Women's Hospital, Boston, MA.

Background: Whereas alveolar rhabdomyosarcoma (ARMS) contains the PAX3FOXO1 or PAX7-FOXO1 fusions that function as aberrant transcription factors (TF) blocking terminal myogenic differentiation, no comparable aberrant $\mathrm{TF}$ has been found in embryonal RMS (ERMS). To identify novel and recurrent oncogenic mutations in RMS, we performed whole exome sequencing (WES) on RMS samples. 
Design: We performed WES on 20 RMS (9 ARMS, 11 ERMS). We also genotyped additional ERMS samples using mass spectrometry-based genotyping (Sequenom). We collected clinicopathologic data and performed survival analyses and functional studies. Results: By WES, 2 ERMS showed the same c. $365 \mathrm{~T}>\mathrm{G}$ somatic point mutation in MYOD1, leading to an L122R substitution. The L122R mutation occurs in the conserved basic region of the DNA-binding domain of MYOD1, at a highly specific residue that is a leucine (L) in all myogenic bHLH TFs (MYOD1, MYF5, myogenin, MRF4) but is an arginine (R) in MYC. We genotyped an additional 93 ERMS for the L122R mutation using Sequenom genotyping. The mutation was found in 8 additional cases, resulting in an overall prevalence of $10 \%(10 / 104)$. The 10 ERMS with the MYOD1 L122R mutation were highly cellular with frequent spindle cell morphology and uniform strong immunopositivity for MYOD1, suggesting, in some cases, an overlap with the recently described adult spindle cell variant of ERMS. Notably, 9 of the 10 ERMS with MYOD1 mutations were diagnosed in adolescence or adulthood (mean age $=25$; median age $=28)$, and they were more likely to arise in the head/neck $(8 / 10 \mathrm{vs} 16 / 80 ; p=0.0003)$. Furthermore, the overall survival with $M Y O D 1$-mutant ERMS was significantly poorer than for ERMS lacking $M Y O D 1$ mutation ( $0 \%$ vs $48 \%$ at $10 \mathrm{yrs}$; $=0.02$ ). We also observed a relationship between $M Y O D 1 \mathrm{~L} 122 \mathrm{R}$ and PIK3CA mutations in 66 cases with genotyping data for both: PIK3CA mutations were seen in 3/10 MYODI L122R cases vs $0 / 56 M Y O D 1$ wild type cases ( $\mathrm{p}=0.003$ ), suggesting possible cooperation between these mutations. Functional studies in $\mathrm{C} 2 \mathrm{C} 12$ cells confirmed that MYOD1 L122R blocks myogenic differentiation and enhances proliferation.

Conclusions: ERMS with MYOD1 L122R represents a novel molecular subset of RMS with distinctive clinicopathologic features that should be considered for high risk protocols and targeted therapeutic development.

\section{An Institutional Review of Non-Epithelial Neoplasms of the Sinonasal Cavity of the Period of $\mathbf{1 0}$ Years at a Tertiary Care Center}

D Kokh, J Choi, AP Burke, JC Papadimitriou. University of Maryland Medical Center, Baltimore, MD

Background: The sinonasal cavities give rise to a heterogeneous group of neoplasms of mesenchymal, neural, epithelial, melanocytic and hematopoietic origin. These account for less than $1 \%$ of all cancers, but their overall frequency is unclear. Recurrence and treatment options are variable depending of the hisopathological profile of the cancer. Design: We retrospectively reviewed all non-epithelial malignancies of the sinonasal cavities at a tertiary care center.

Results: Of 720 sinonasal neoplasms from 2003-2013,37 cases (5\%) were non-epithelial neoplasms. These were comprised of sarcomas $(n=11)$, mucosal melanomas $(n=8)$, benign neoplasms $(n=7)$, lymphomas $(n=2)$, and germ cell tumors $(n=1)$. Of the 37 study cases, 9 arose from bone. Of the sarcomas, four were chondroblastic osteosarcomas of the maxillary bone and the nasal cavity, exclusively in males 33-62 years old, with two tumors being recurrent. Alveolar type rhabdosarcoma for the ethmoid and maxillary sinuses was seen in one male and one female 52 and 57 years of age respectively; recurrence was seen in one patient. One of each of the following malignant soft tissue tumors were seen: a low grade fibromyxoid sarcoma of the nasal cavity in a 23 year old male with no known recurrences, a spindle cell sarcoma of the frontal, maxillary and sphenoid sinuses in a 58 year old male, a myxoid liposarcoma of the maxillary tuberosity in a 13 year old male with known multiple recurrences, and a angiosarcoma of the nasal septum in a 78 year old male. Of the borderline malignancies, the following tumors were observed: a hemangiopericytoma of the nasal cavity in a 49 year old male with no recurrences, a desmoplastic fibroma of the maxilla in a 50 year old male, and five cases of fibrous dysplasia occurring mostly in the maxillary and ethmoid sinus in two females and three males 15-56 years old, with most patients experiencing multiple recurrences. Mucosal malignant melanomas of the nasal cavity, maxillary and ethmoid sinuses were seen in eight patients five of which were females and three were males 51-97 years old with multiple recurrences in 6 cases. Two large B-cell lymphomas were observed in a male and a female patient 52-57 year of age. There was a case of a nasal cavity teratoma in a 10 year old boy with numerous recurrences and eventual death. Conclusions: In our study non-epithelial neoplasms constituted $5 \%$ of primary sinonasal tumors, malignant in their majority, often aggressive with multiple recurrences, heterogeneous, with soft tissue sarcomas most frequent.

\section{Evaluation of CD24 Expression in Mesenchymal Neoplasms: A Specific Biomarker for Low Grade Fibromyxoid Sarcoma?}

G Kristiansen, SC Smith, A McDaniel, PW Harms, JB McHugh, D Thomas, DR Lucas, RM Patel. Universitätsklinikum Bonn, Bonn, Germany; Cedars-Sinai Medical Center, Los Angeles, CA; University of Michigan, Ann Arbor, MI.

Background: The membrane glycoprotein CD24, associated with metastasis and stem cell-like properties, has been shown to be expressed in numerous carcinomas. Recent molecular studies suggest this protein may be induced by the recurrent FUS-CREB3L2/ L1 fusion oncogenes of low grade fibromyxoid sarcoma/Evans tumor/hyalinizing spindle cell tumor with giant rosettes (LGFMS). CD24 has not been evaluated in sarcomas, particularly using new specific protocols detecting the peptide backbone rather than the sugar epitopes that decorate this protein.

Design: Whole sections of confirmed LGFMS $(\mathrm{N}=8)$ and whole sections and tissue microarrays of lesions in its differential $(\mathrm{N}=136)$ as well as other predominantly high grade lesions $(\mathrm{N}=120)$ were immunostained by standard means using a recently validated monoclonal antibody detecting CD24 protein. Tissues were scored by proportion of viable lesional cells positive for CD24 $(0-3+)$, with intravascular granulocytes as positive internal control. Differences in proportions of lesions staining positive $(<1+)$ with CD24 were tested by Fisher's Exact Test.

Results: Of LGFMS, 7/8 showed diffuse $3+$ positivity for CD24, cytoplasmic and membranous. Of lesions in the differential, 0/57 dermatofibrosarcoma protuberans, 0/46 fibromatoses, $0 / 7$ nodular fasciitides, and $0 / 29$ solitary fibrous tumors were positive
$(\mathrm{P}<0.0001)$. Of high grade lesions, $1 / 10$ undifferentiated pleomorphic sarcomas, $6 / 28$ uterine and 5/57 non-uterine leiomyosarcomas, 0/38 Ewings family tumors, 0/6 CICDUX4 sarcomas, 8/33 Synovial sarcomas, 0/27 malignant peripheral nerve sheath tumors, and 2/18 gastrointestinal stromal tumors were positive for CD24.

Conclusions: CD24 expression was robust in LGFMS but undetected in other lesions in the differential, with scattered expression in high grade sarcomas and other lesions studied. These findings suggest that CD24 induction is a specific feature of the molecular alterations pathognomonic for LGFMS and may by useful as a diagnostic surrogate.

71 Myxofibrosarcoma:Reappraisal of Clinical Outcome Including Predictive Effects of Histologic Variables

\section{Langston, E Lawson, F Eilber, D Sarah. UCLA, Los Angeles, CA}

Background: Myxofibrosarcoma (MFS), an infiltrative sarcoma, typically occurs in superficial tissues of elderly patients' extremities. Historically, up to $50 \%$ have local recurrence (LR) and up to $35 \%$ develop distant metastases (DM). Prior grading schemes have not predicted clinically aggressive behavior. We studied a series of MFS, all resected in an oncologic fashion at a single institution, to determine if historic LR/DM rates persist and to identify histologic predictors of clinical outcome

Design: Review of our Pathology database identified 46 MFS cases resected between 2003 and 2013 which underwent definitive excision at our institution. Some cases had prior outside diagnostic biopsy, all reviewed at our institution. Only cases with slides of the original tumor available for review were included. Histologic (ie, margin status, percent non-myxoid stroma, grade by the French system) and clinical (ie, treatment received, LR, DM, status) parameters were assessed.

Results: Our 46 cases ( 22 male, 24 female) mainly arose in the elderly (mean 66 years, range 42-89) with one tumor in a 29 year old female. Tumors ranged from $2-22 \mathrm{~cm}$. Initial resection margins were positive in $23(53 \%) .5$ patients required 2 re-excisions to achieve negative margins on the initial tumor, including one that required amputation. $12 / 46$ initial tumors $(26 \%)$ had less than $25 \%$ non-myxoid stroma. Clinical follow up (mean 48 months) was available for $35 / 46(76 \%) .1 / 35(3 \%)$ died of disease at 24 months. $8 / 35$ (23\%) had LR (mean 18 months, range 6-47 months); 6/8 with LR had negative margins on initial excision, including 4 patients with $>0.5 \mathrm{~cm}$ margins, $2<0.5 \mathrm{~cm}$ and $2<0.1 \mathrm{~cm} .12 / 35(34 \%)$ had more than one LR. 2 patients with LR required amputation for local control. 7/35 (20\%) developed DM (mean 20 months, range $4-77$ ) to the lung (4), lymph nodes (2), axilla (1), thigh (1); 1 patient had 2 DM. By the French system $6 / 7(86 \%)$ patients with DM had high grade and 1/7 (14\%) intermediate grade tumors; all $(7 / 7(100 \%))$ patients with DM had $>25 \%$ non-myxoid stroma.

Conclusions: Our series of MFS indicates that historic rates of LR persist, although rates of DM are lower. MFS is a clinically aggressive tumor with LR in $8(23 \%)$ patients and DM in $7(20 \%)$ in our series. All patients with DM had $>25 \%$ non-myxoid stroma in the initial tumor; the initial tumor was also high grade by the French system in 6/7. We are expanding our series through identification of additional cases with longer follow up to further investigate the effect of non-myxoid stroma and French grade on clinical prognosis.

72 MYC Expression in Sporadic and Radiation-Associated Sarcomas SM Lee, L Huo, AJ Lazar, W-L Wang. University of Texas MD Anderson Cancer Center, Houston, TX

Background: The $M Y C$ proto-oncogene( $8 \mathrm{q} 21$ ) regulates genes involved in cell division, cell growth, and apoptosis. Amplification of $M Y C$ resulting in its overexpression has been described in radiation associated mammary angiosarcoma. However, the role and prevalence of Myc expression in sporadic and other radiation-associated sarcomas has not been extensively investigated.

Design: 53 radiation-associated sarcomas(49 undifferentiated pleomorphic sarcomas (UPS), 2 liposarcomas, and 2 osteosarcomas), 119 sporadic sarcomas(12 liposarcomas, 18 synovial sarcomas, 4 leiomyosarcomas, 71 UPSs, 14 unclassified sarcomas), and 27 radiation associated angiosarcomas(for comparison) were analyzed using formalin-fixed paraffin-embedded tissue microarrays. Immunohistochemical studies were performed using the rabbit monoclonal anti-Myc antibody to the $\mathrm{N}$ terminus of human Myc (Y69; Ventana Medical Systems, Tucson, AZ, USA). Immunoreactivity was graded for intensity(weak, moderate, and strong). Any labeling was considered positive.

Results: In sporadic sarcomas, MYC reactivity was observed in 87 of 119 cases (73\%); 17 of which had moderate to strong labeling. These were seen in UPS(12), synovial sarcoma(2), unclassified sarcoma(2), and leiomyosarcoma(1). In radiation-associated sarcomas(non-angiosarcoma), 22 of 53 cases( $47 \%$ ) had MYC expression, 17 cases with weak and 7 cases, all UPS, with moderate to strong labeling. In contrast, MYC was expressed in 26 of $27(96 \%)$ radiation-associated angiosarcoma, with predominantly diffuse and strong labeling (20/26).

Conclusions: Variable MYC expression, including occasionally moderate to strong labeling, can be seen in sarcomas other than angiosarcomas including other radiationassociated sarcomas though less than radiation associated angiosarcomas. These findings suggest that MYC may play a role in tumoriogenesis in sarcomas and may serve as a potential therapeutic target. The mechanism of overexpression in these tumors has yet to be elucidated.

\section{$73 \quad$ MYC Expression in Mammary Vascular Tumors}

SM Lee, L Huo, AJ Lazar, W-L Wang. University of Texas MD Anderson Cancer Center, Houston, TX

Background: Post radiation angiosarcomas have high level $M Y C$ amplification resulting in its overexpression. Previous studies have shown that MYC immunohistochemical studies are useful in distinguishing post radiation mammary angiosarcoma from 
atypical vascular proliferation. However, the prevalence of MYC expression and its diagnostic utility in evaluating primary mammary vascular tumors have not been extensively studied.

Design: Formalin-fixed paraffin-embedded tissue microarrays and whole tissue sections of 20 mammary hemangiomas, 12 primary mammary angiosarcomas and 24 post radiation mammary angiosarcomas were analyzed by immunohistochemistry using monoclonal anti-Myc antibody to the N terminus (Y69; Ventana Medical Systems, Tucson, AZ, USA). Any amount of staining in the tumor cells was considered positive. The staining intensity was categorized as weak, moderate and strong.

Results: The staining results were summarized in Table 1. Most of the post radiation angiosarcomas had moderate to strong Myc staining (18/24, 75\%). In contrast, only $33 \%$ (4/12) of primary mammary angiosarcomas had moderate to strong staining. Although none of the hemangiomas had strong staining, 2 had moderate staining. Weak staining was observed in tumors of all 3 types.

\begin{tabular}{|c|c|c|c|}
\hline & $\begin{array}{l}\text { Primary } \\
\text { hemangioma }\end{array}$ & $\begin{array}{l}\text { Primary } \\
\text { angiosarcoma }\end{array}$ & $\begin{array}{l}\text { Post radiation } \\
\text { angiosarcoma }\end{array}$ \\
\hline$\#$ of cases positive staining/total \# of cases & $10 / 20$ & $10 / 12$ & $23 / 24$ \\
\hline$\#$ of cases with weak/moderate/strong labeling & $8 / 2 / 0$ & $6 / 3 / 1$ & $5 / 6 / 12$ \\
\hline
\end{tabular}

Conclusions: In breast, the sensitivity of Myc immunohistochemical staining is lower in primary angiosarcomas compared with post radiation angiosarcomas. Strong Myc staining can be potentially used to distinguish angiosarcomas from hemangiomas. While weak staining is not diagnostically helpful, moderate Myc staining in mammary vascular tumors need to be interpreted with caution because it can be seen in a small proportion of hemangiomas.

74 Characterization of the Clinical, Biological, and Therapeutic Relevance of EZH2 Overexpression in Myxofibrosarcoma

C-F Li, H-Y Huang. Chi-Mei Medical Center, Tainan, Taiwan; National Health Research Institutes, Tainan, Taiwan; Southern Taiwan University of Science and Technology, Tainan, Taiwan; Kaohsiung Chang Gung Memorial Hospital and Chang Gung University College of Medicine, Kaohsiung, Taiwan.

Background: Myxofibrosarcoma is genetically complex and insufficiently characterized in molecular determinants of clinical aggressiveness. By reappraising the public transcriptome (GSE21122), EZH2 was identified as a top-ranking differentially upregulated gene among those regulating chromatin organization (GO:0006325) in myxofibrosarcoma tissues.

Design: To validate the pathogenetic role of EZH2 in myxofibrosarcoma, mRNA abundance and protein expression of EZH 2 were determined in independent samples by branched-chain DNA (bDNA) and immunohistochemical assays, yielding 40 and 87 informative cases, respectively. In vitro, RNA interference and DZNep treatment (targeting EZH2 associated PRC2 complex) were applied in EZH2-expressing myxofibrosarcoma cell lines to evaluate for the biological functions and therapeutic relevance of EZH2. The in vivo effect of DZNep treatment was examined in xenograft models established from two myxofibrosarcoma cell lines.

Results: EZH2 protein overexpression was associated with higher histological grade $(\mathrm{p}=0.002)$, worse disease-specific and metastasis-free survival (both $\mathrm{p} \leq 0.0001$ ), and mRNA upregulation $(\mathrm{p}=0.010)$. In all myxofibrosarcoma cell lines, stable EZH2 knockdown resulted in impaired cell proliferative, migratory, and invasive capabilities with concomitant reductions in anchorage-independent colony formation and wellformed endothelial tubes. In vitro, DZNep caused dose- and time-dependent cytotoxicity and downregulated PRC2-associated proteins with induction of cellular apoptosis and attenuation of EZH2 promoter transactivity. The dose-dependent therapeutic efficacy of DZNep was further corroborated in vivo in both xenograft myxofibrosarcoma models. Conclusions: EZH2 overexpression plays an oncogenic role in myxofibrosarcoma pathogenesis and promotes tumor growth, migration/invasion, and angiogenesis, hence conferring clinical aggressiveness and representing a novel therapeutic target of DZNep therapy.

75 MicroRNA Expression Profiling Identifies Potential Prognostic Biomarkers in Osteosarcoma

$Y$ Liang, $K$ Wani, L Long, MT Deavers, AJ Lazar, KD Aldape, W-L Wang. UT MD Anderson Cancer Center, Houston, TX

Background: The most important post-adjuvant prognostic factor of osteosarcoma (OSA) is pathologic response to chemotherapy, evaluated only by a crude assessment of post-treatment necrosis ( $>90 \%$ necrosis predicting a better outcome). Improved prognostic markers are needed to predict recurrences, metastases, and therapeutic responses, ideally prior to adjuvant therapy. MicroRNAs (miRNA) are non-coding RNAs with regulatory functions and their differential expressions have been described in OSA. We compared the miRNA expression profiles in osteosarcomas from patients with good and poor outcomes to identify specific miRNAs with useful prognostic associations on formalin-fixed paraffin embedded (FFPE) tissue.

Design: RNA was extracted from 20 decalcified FFPE pretreatment high-grade OSA biopsy specimens: 10 tumors from patients with disease-free survival in follow-up (good outcome), and 10 tumors from patients with recurrences and metastases (poor outcome). MiRNA expression profiling was performed using a real-time PCR based platform on a Taqman Human miRNA array card containing 378 annotated miRNAs. Expression was normalized to housekeeping miRNAs. Expression level changes of 2.3 folds were considered significant. Two sample t-tests were used on a probe-by-probe basis to compare the two groups with $p$-value $<0.05$ being considered statistically significant. Results: The patients in good outcome group had a median clinical follow-up of 90.5 (range 51.8-113) months without metastases. Within the poor outcome group, all patients had distant metastasis with nine expired at a median survival of 29 (range 13.7-84.9) months and one under palliative care at 28 month follow-up. Two sample t-tests identified the expression of 3 miRNAs: miR-150(7.8 fold), miR-494(5.9 fold), and miR-451(4.8 fold), were significantly increased in the poor outcome group. The expressions of miR-133a miR-376a, miR-410, miR-127, miR-370, and miR-483-5p also treaded toward significance. In contrast, 9 miRNAs: miR-425-5p(16.2 fold), miR-10b(9.8 fold), miR-22(8.4 fold), miR-18a(8.1 fold), let- $7 \mathrm{a}(7.8$ fold), miR-140(6.2 fold), miR-186(4.9 fold), miR-361(3.1 fold), and miR-148a(2.6 fold) expressions were significantly higher in the good outcome group.

Conclusions: We identified a subset of miRNAs that differentiated between OSAs with good and poor outcomes by using formalin fixed paraffin-embedded tissue, irrespective of tumor necrosis. Many of these miRNAs are known to regulate genes associated with apoptosis, metastasis, and cell growth including the PI3kinase pathway. miRNA show promise as useful clinical prognostic biomarkers.

76 Desmoid-Type Fibromatosis: Radiologic-Pathologic Correlation and Clinical Predictors of Recurrence in an 18-Year Retrospective Cohort JG Mantilla Arango, EM Shustef, E Villanueva-Siles. AECOM/Montefiore Medical Center, Bronx, NY.

Background: Desmoid-type fibromatosis is a relatively rare, locally aggressive, fibrous neoplasm with an extensive differential diagnosis. These tumors are estimated to have a $20-45 \%$ risk of recurrence. Higher risk of recurrence has been associated with age, tumor size, and mutation of the CTNNB1 gene. We aimed to establish specific criteria to predict the risk of recurrence and help guide individualized surveillance and treatment. Design: We conducted a retrospective cohort study of 51 patients diagnosed with desmoid-type fibromatosis between 1995 and 2008. The subset of patients and clinical information was extracted from our clinical information system using Clinical Looking Glass; Emerging Health Information Technology, Yonkers, NY. We reviewed initial imaging modalities, diagnoses, and recurrence rates 5-13 years post-diagnosis. Risk of recurrence was determined based on age, sex, race, anatomic site, and surgical margin status.

Results: Of 51 patients, 18 developed post-operative recurrences during follow-up (34.6\%). Mean time to recurrence was 18.3 months. Females were more frequently affected $(68.6 \%)$, and also showed a higher recurrence rate $(42.9 \%)$ compared to their male counterparts $(18.8 \%)$. The median age at diagnosis was 37.5 years, with $72 \%$ of patients below age 50. Blacks were more commonly affected (39.2\%), followed by Hispanics $(23.5 \%)$ and Caucasians $(21.6 \%)$. The latter showed the lowest rate of recurrence. The abdominal wall was most frequently affected $(30.8 \%)$, followed by mesentery $(13.5 \%)$ and upper extremities $(13.5 \%)$. Tumors located in the upper extremities showed a markedly increased rate of recurrence, as well as those with positive and unknown surgical margin status ( 35 and $45 \%$, respectively). Four patients developed different primary neoplasms during follow-up and two patients had separate metachronous fibromatoses. One patient had a prior diagnosis of adenomatous polyposis of the colon. 32 patients underwent imaging studies prior to surgery. The majority received a CT scan as the initial imaging modality (68.5\%), followed by MRI $(21.8 \%)$. The most common radiologic diagnosis was unspecified mass/neoplasm (31.3\%), followed by sarcoma $(28.1 \%)$, hematoma $(21.8 \%)$ and desmoid-type fibromatosis $(21.8 \%)$.

Conclusions: In our cohort, desmoids were observed most commonly in women and patients below age 50. Lesions occurring in women, in the upper extremities or with positive surgical margins showed a higher rate of recurrence. Radiologic diagnosis was often nonspecific. A larger study is necessary to better determine relative risk for recurrence.

77 The Expression of STAT6 in Mesenchymal Tumors: An Immunohistochemical Study with an Emphasis on the Morphological Spectrum of Solitary Fibrous Tumor

A Matsuyama, T Kasai, R Shibuya, E Shiba, M Nakamoto, $M$ Hisaoka. University of Occupational and Environmental Health, Kitakyushu, Fukuoka, Japan.

Background: The soft tissue tumors previously designated by Stout as hemangiopericytoma (HPC) have been classified into several categories of tumors including solitary fibrous tumor (SFT), and the term "HPC" has been preserved only in HPC of central nervous system (CNS) and sinonasal-type HPC in the recent WHO classifications of tumors. In 2013, solitary fibrous tumor has been demonstrated to constantly harbor the NAB2-STAT6 fusion gene. The subsequent immunohistochemical and molecular study showed that SFT and HPC of CNS share the fusion gene and thus they are considered to constitute variants of a single tumor entity. It is recommended to re-evaluate the histological spectrum of SFTs by detection of the STAT6 rearrangement in soft tissue tumors including sinonasal-type HPC.

Design: We examined immunohistochemical expression of STAT6 in 400 mesenchymal tumors including 29 SFTs (2 fat-forming type, 3 giant cell angiofibroma and 3 malignant), 2 sinonasal-type HPC and 4 myopericytomas. Only intranuclear staining was considered to be positive.

Results: Immunohistochemically, 24/29 STFs were diffusely and intensely positive for STAT6. STAT6-negative SFTs included 2 fat-forming types, 2 giant cell angiofibromas and 1 mitotically active SFT. STAT6 was also expressed in 1 myopericytoma, which focally showed characteristic concentric growth of tumor cells and was diffusely immunoreactive to actins but negative for $h$-caldesmon. STAT6 was only focally and weakly stained in other 5 tumors (2/25 spindle cell lipomas, $2 / 28$ nodular fasciitis, $1 / 7$ low grade fibromyxoid sarcoma). On the other hand, the other tumors including tumors simulating SFT or with a hemangiopericytomatous appearance such as angiomyofibroblastoma, cellular angiofibroma, myofibroma and sinonasal-type HPCs were all negative for STAT6.

Conclusions: Our study suggests that STAT6 is a useful immunohistochemical marker for a diagnosis of SFT. Histological variants of SFT include giant cell fibroblastoma, and fat-forming SFT and sinonasal-type HPC may be distinctive entities of tumors 
based on the status of the STAT6 expression. A subset of SFT and myopericytoma may share the morphological and immunohistochemical features including a characteristic concentric growth of actin-positive myoid cells.

\section{A Subset of Solitary Fibrous Tumors Express Nuclear PAX8 and PAX2: A Potential Diagnostic Pitfall}

A McDaniel, N Palanisamy, S Smith, D Robinson, Y-MWu, A Chinnaiyan, JK Greenson, LP Kunju. University of Michigan, Ann Arbor, MI.

Background: Solitary fibrous tumor (SFT) is an uncommon mesenchymal neoplasm of probable fibroblastic origin with widespread anatomic distribution which can be difficult to diagnose in limited samples. We recently encountered a diagnostically challenging fine needle aspirate of a pancreatic mass, which was initially incorrectly diagnosed as metastatic renal cell carcinoma based on strong PAX8 expression by immunohistochemistry (IHC). After Whipple resection, morphologic features along with additional IHC (CD34 positivity) identified this lesion as a SFT. PAX8 and PAX2 are transcription factors commonly used as markers of primary and metastatic renal and Mullerian malignancies. To date, no series has investigated the prevalence of PAX8 or PAX2 expression in SFT.

Design: IHC for PAX8 (Cell Marque) and PAX2 (Invitrogen) was performed on from 37 biopsy and resection specimens of SFT from the pleura and thorax $(n=12)$, head and neck $(n=20)$, pelvis $(n=3)$, extremities $(n=1)$, and retroperitoneum $(n=1)$. The mean patient age was 55.1years (15-86). Confirmatory CD34 staining was present in 21 of 23 cases $(93 \%)$. Tumors ranged in size from 1.2 to $27 \mathrm{~cm}$. Six tumors were considered histologically malignant and six tumors were recurrences of previously incomplete resections. Staining intensity for both PAX8 and PAX2 $(0=$ no stain, $1+=$ unequivocal but weak, $2+$ moderate, $3+$ strong $)$ and extent $(<50 \%$ of nuclei stained $=$ focal, $>50 \%$ of nuclei stained $=$ diffuse) was recorded in each case, with only cases that had $2+$ or $3+$ staining scored as positive.

Results: In our cohort, nuclear PAX8 staining was observed at least focally in $29.7 \%$ (11 of 37) SFT cases; additionally, nuclear PAX2 staining was positive in $16.7 \%$ (6 of 36 cases) SFTs. All cases positive for PAX2 were also positive for PAX-8; while five cases of PAX8 positive SFTs showed no nuclear staining for PAX-2. For both PAX8 and PAX2 positive cases, half $(50 \%)$ of the cases showed diffuse expression across the tumor, while the other half showed focal expression. We found no correlation between PAX8 and PAX2 positivity and patient age, tumor size, site, cellularity, malignant categorization, or recurrence status.

Conclusions: A substantial minority of SFTs demonstrate nuclear expression of PAX8 and PAX2 by IHC, frequently with strong, diffuse expression. These findings present a diagnostic pitfall when evaluating possible metastases from the kidney or gynecologic tract, particularly when the primary tumor shows sarcomatoid or spindle cell morphologies.

\section{Osteosarcoma of the Foot: A Review of 15 Cases}

T Mishra, A Puri, S Desai, B Rekhi, A Gulia, J Bajpai, $N$ Jambhekar. Tata Memorial Hospital, Mumbai, Maharashtra, India.

Background: Osteosarcoma (OS) is the commonest primary tumour of the bone, next only to multiple myeloma. The foot is an uncommon site comprising only $0.17-2.08 \%$ of all OS. This is a review of 15 OS of foot accrued in the Pathology Department of a tertiary cancer hospital over ten years.

Design: Of the 15 cases of OS of the foot between 2003 and 2013, 10 were primarily treated at this hospital, and five were pathology consults. All clinical, radiological and treatment details were abstracted from the charts. All histology material (slides and paraffin blocks) were re-examined.

Results: The 15 cases comprised nine males and six females. The youngest patient was an eight-year old female and the oldest was a 68-year old male (mean age 32 years). All patients had pain and persistent swelling of the affected area, but none had a fracture. The affected bones were calcaneum: 8 cases, talus: 4 , metatarsal: 1 and soft tissue: 2 cases. Radiological data was available in 12 cases, of which 11 were reported as either a "primary bone malignancy" or features suggestive of malignancy. In the single exception, the differential was between a chronic fungal infection or an OS. Histologically all cases were conventional high grade OS, of which five were Chondroblastic, three were Osteosclerotic and one was a Giant Cell Rich OS. Nine of the ten patients treated at this hospital received neoadjuvant chemotherapy followed by below knee amputation. The tenth patient had an extraskeletal OS and underwent a wide local excision. Follow-up was available in seven patients (duration 6 to 60 months). Five patients developed metastasis, of which one had metastasis to inguinal lymph nodes at presentation; the remaining developed metastasis to lung and liver, lung, inguinal lymph nodes and lung at $12,15,18$ and 25 months respectively after treatment. Conclusions: These 15 cases constituted only $0.62 \%$ of all OS accrued in this hospital in a decade. The mean age of foot OS in this study was 32 years, whereas conventional OS mostly affects children and adolescents. Almost half of foot OS affected the calcaneum followed by talus, a finding corroborated in literature. In the largest series of foot OS (Mayo clinic), the majority ( 44 of 52 cases) were high grade, similar to our observation. However, Biscaglia et al (1998) found five of 12 tumors to be low grade. To conclude, primary OS of foot is a rare tumour, which needs to be differentiated from the several more frequent non-osseous lesions affecting the foot.
80 Immunohistochemical and RT-PCR Analyses of NAB2-STAT6 Fusion Gene Products Reveal Immunodetection of Nuclear STAT6 Protein as a Practical Diagnostic Tool for Solitary Fibrous Tumor

TMotoi, I Kato, Y Fukuda, M Ogawa, H Koiwai, S Horiguchi, T Goto, T Hishima. Tokyo Metropolitan Cancer and Infectious Diseases Center Komagome Hospital, Tokyo, Japan. Background: Solitary fibrous tumor (SFT) is a fibroblastic tumor that occasionally poses diagnostic challenges. Recently, NAB2-STAT6 fusion gene derived from the genomic inversion of $12 \mathrm{q} 13$ locus was identified as a constant abnormality of SFTs. In order to utilize the NAB2-STAT6 product as a practical diagnostic tool, we explored its status by using immunohistochemistry and RT-PCR.

Design: Fifteen typical SFTs ( 5 pleural and 10 extrapleural) were immunostained by monoclonal NAB2 and polyclonal STAT6 antibodies. Non-SFT tumors (NTs) that may clinically or histologically mimic SFTs were also examined; they included 16 malignant mesotheliomas, 20 fibroblastic tumors of various types, 5 fibrous histiocytomas (FH), 16 undifferentiated pleomorphic sarcomas (UPS), 17 CD34-positive tumors, 16 miscellaneous spindle cell tumors. The nuclear and cytoplasmic stainings were evaluated separately, and they were scored as $2(\geq 50 \%$ of tumor cells stained with strong intensity), $1(5-<50 \%$ stained with any intensity), or $0(<5 \%$ stained with weak intensity). RT-PCR and direct sequencing were performed using primer sets that were designed to detect previously reported 4 major fusion transcripts.

Results: NAB2 was exclusively localized in the nuclei of positive cells, whereas STAT6 was localized in the nuclei and/or the cytoplasms. Score 2 positivity for NAB2 and nuclear STAT6 was observed in all SFTs. None of NTs showed score 2 positivity for nuclear STAT6, while score 2 NAB2 reactivity was also found in NTs $(37.8 \%, 34 / 90)$ including $68.8 \%(11 / 16)$ of UPSs and $80 \%(4 / 5)$ of FHs. When the reactivity was categorized into positive (score 2 ) or negative (score 0 or 1 ), the sensitivity/specificity for NAB2 and nuclear STAT6 was $100 \% / 62.2 \%$ and $100 \% / 100 \%$, respectively.By RT-PCR, fusion transcripts were detected in $66.7 \%(10 / 15)$ of SFTs, including 7 exon 4-3, 1 exon 6-17, 2 exon 6-18, and 0 exon 7-3 fusion transcripts

Conclusions: Immunohistochemical detection of nuclear STAT6 is the most reliable and practical adjunct for diagnosing SFTs because of a fairly consistent staining pattern regardless of the tumor sites, histology, and fusion transcript subtypes. Concomitant nuclear overexpression of NAB2 and STAT6 presumably indicates the fusion gene products. By contrast, the fusion transcripts were detectable by RT-PCR in only a limited number likely due to the variety in exon combinations.

81 Impacts of a Systematic Histological Review for Desmoid Tumors: About a Series of 735 Cases Reviewed in $\mathbf{4 2}$ Months

A Neuville, A-V Decouvelaere, D Ranchere-Vince, P Terrier, F Collin, J-M Coindre. Institut Bergonie, Bordeaux, France; Centre Léon Bérard, Lyon, France; Institut Gustave Roussy, Villejuif, France; Centre Geoges-François Leclerc, Dijon, France.

Background: Desmoid tumors (DT) are rare tumors which are locally aggressive and for which the treatment is changing towards a "wait and see" strategy demanding a firm diagnosis on core needle biopsy. Previous publications on DT reported biased series from specialized centers.

Design: A national network of pathologists has been set up in France for a systematic review of every new sarcoma, GIST and DT. Moreover, a molecular test is systematically performed for every suspicion of translocation, $M D M 2$ amplification or specific mutation for GIST and DT. The following data are collected in a shared database (https://www. rreps.sarcomabcb.org): sex, age, location, depth and size of tumor, date and type of sampling, type and result of molecular test and histotype.

Results: From January $1^{\text {st }} 2010$ to June $30^{\text {th }} 2013$, 735 DT have been collected, representing $4.9 \%$ of all registered mesenchymal tumors. They occurred in 478 women and 257 men (mean 44 years, range $1-90$, with 31 patients $<15$ ). A previous history of Gardner syndrome was present in 21 cases. Tumor location was abdominal wall (40\%), other trunk wall $(21 \%)$, limbs $(15 \%)$, intra-abdominal $(11 \%)$ and other $(13 \%$ with 24 cases in the breast) in women and intra-abdominal (45\%), abdominal wall (12\%); other trunk wall $(18 \%)$, limbs $(14 \%)$ and others $(11 \%)$ in men. Type of sampling for the diagnosis has been changed from $22 \%$ of core needle biopsies in 2010 to $34 \%$ in 2012. Diagnostic discordance has been observed in $20 \%$ of cases with 86 false negative and 76 false positive diagnoses of DT. A mutational analysis of CTNNBI has been performed for $92 \%$ of cases and was positive for $79 \%$ of cases.

Conclusions: This analysis shows the importance of a structured network for the final diagnosis of DT, the feasibility of an almost systematic use of molecular pathology even on core needle biopsies and a more accurate repartition of DT according to the location since the network reviews about $90 \%$ of expected cases of sarcomas, GIST and DT in France.

Comprehensive Genomic Evaluation of Osteosarcoma with a Targeted Next Generation Sequencing Assay Reveals Frequent Actionable Genomic Abnormalities and Potential New Routes to Targeted Therapies GG Niu, RN Al-Rohil, M Vanushkina, CE Sheehan, T Nazeer, M DiCaprio, G Palmer, SM Ali, D Morosini, VA Miller, PJ Stephens, JS Ross. Albany Medical College, Albany, NY; Foundation Medicine Inc, Cambridge, MA

Background: Although current therapies have significantly reduced disease-related mortality, recurrent and metastatic osteosarcoma (OS) has a poor prognosis. In this study, comprehensive genomic profiling of relapsed/metastatic OS samples by next generation sequencing (NGS) was performed to identify genomic-derived drug targets of therapy not tested for in routine clinical practice.

Design: Hybridization capture of 3,769 exons from 236 cancer-related genes and 47 introns of 19 genes commonly rearranged in cancer was applied to $\geq 50 \mathrm{ng}$ of DNA extracted from 42 FFPE samples of OS and sequenced to high, uniform coverage (mean depth 898X) in a CLIA lab (Foundation Medicine). Genomic alterations (base 
substitutions, small indels, rearrangements, copy number alterations) were determined and then reported for these patient samples. Actionable GA were defined as those targeted by anti-cancer drugs on the market or in registered clinical trials (CT).

Results: There were 17 female and 25 male patients with a median age 32 years (range 9-73 years). There were 4 intermediate grade and 38 high grade tumors. Five OS were Stage II, 3 were stage III and 34 were Stage IV at time of sequencing. Twenty six (23 from bone, 3 from soft tissue,) were primary tumors and 16 were metastases biopsies. A total of $130 \mathrm{GA}$ were identified in $36 / 42(86 \%)$ with an average of $3.1 \mathrm{GA}$ per tumor. The most common non-actionable GA were mutations in TP53 (38\%), RB1 (21\%), and DNMT3A (7\%) and amp in MYC (21\%). Twenty eight (67\%) OS harbored at least 1 actionable GA with an average of 1.62 actionable GA per patient including: mutation/ homozygous deletion in CDKN2A/B (24\%) PTEN (10\%) and PIK3CA (7\%); amp in PDGFRA (14\%), MCL1 (14\%), KIT (12\%), KDR (12\%), CCNE1 (12\%) CDK4 (5\%), and $M E T(5 \%)$. A long tail of actionable alterations in a single case included: $B R C A 2$, CCND1, CCND2, FGFR1, GNAS, FBXW7, MAP2K2, MDM2, PTCH1, RAD50, RICTOR and TSC1.

Conclusions: Two-thirds of OS harbored actionable GA which has the potential to influence and personalize therapy selection. The diverse list of altered genes and involved genomic pathways indicate that a broad diagnostic assay is needed to maximize targeted therapeutic options for each OS patient. Thus, given the limited current treatment options for patients with metastatic OS, comprehensive NGS-based genomic profiling has the potential to identify new treatment paradigms and meet an unmet clinical need for this disease.

$83 \quad$ Epidemiological Comparison of 9,200 Primary Bone Tumors Treated at Ji Shui Tan Hospital in Beijing, China with 11,839 Patients at Mayo Clinic, Rochester, MN, US

$X$ Niu, $H X u$, CY Inwards, Y Li, $Q$ Zhang, Y Ding, D Letson, MM Bui. Beijing Ji Shui Tan Hospital, Peking University, Beijing, China; Mayo Clinic, Rochester, MN; Beijing Ji Shui Tan Hospital, Beijing, China; H. Lee Moffitt Cancer Center and Research Institute, Tampa, FL.

Background: Although primary bone tumors are extremely rare, current literature shows that there are worldwide variations in the epidemiological characteristics of this diverse diasease. Mayo Clinic data, published in the Dahlin's Bone Tumors 6th edition, is the largest and frequently cited regarding to the epidemiological characteristics of primary bone tumors from US. However, there was no parallel published data in China which precludes a direct comparison.

Design: This study utilized a newly created innovative online Bone and Soft Tissue Tumor Database of JST (http://www.sarcoma-jst.org), which encompasses the epidemeological data of 40 years (1973-2012). A comparison study between 9,200 patients treated at Beijing Ji Shui Tan Hospital (JST) and 11,839 patients treated at Mayo Clinic (MC), Rochester MN, US was conducted to identify any potential epidemiological differences. The patients' age and sex, the tumors' site and histologic diagnosis were analyzed. Statistical analysis was performed with the SPSS software (version 20.0; IBM).

Results: Giant cell tumor and osteosarcoma had significantly higher incidences in the JST than the Mayo Clinic patients $(\mathrm{P}<0.001)$. However, JST patients had a significantly lower incidence of Ewing sarcoma, chordoma, fibrosarcoma, myeloma, and malignant lymphoma $(\mathrm{P}<0.001)$. For most benign and malignant bone tumors, the Chinese cohort showed a more distinct male predominance than the US cohort. In the JST patients, malignant bone tumors demonstrated a mono-modal age distribution with the peak incidence in the second decade of life (36.6\%), while a bimodal age distribution in the MC cohort with an initial peak in the second decade of life $(21.0 \%)$ and second peak between the fifth and seventh decades of life. Predilection to femur and tibia was also found in the JST patients $(\mathrm{P}<0.001)$.

Conclusions: This is the first study demonstrating the epidemiological differences of primary bone tumors between the largest Chinese and US cohorts. The result may guide future studies to better understand the contributing genetic and enviromental factors of these tumors.

84 Microarray-Based DNA Methylation Study of Ewing's Sarcoma of Bone

B-J Noh, H-R Park, W-W Jung, H-S Kim, Y-K Park. College of Medicine, Kyung Hee University, Seoul, Republic of Korea; College of Medicine, Hallym University, Anyang, Gyeonggi-do, Republic of Korea; College of Health Science, Korea University, Seoul, Republic of Korea.

Background: Alterations in DNA methylation patterns are a hallmark of malignancy. However, most epigenetic studies of Ewing's sarcoma have focused on the analysis ofonly a few candidate genes and comprehensive studies were thus lacking and necessary. The aim of this study was to identify novel methylation markers in Ewing's sarcoma using microarray analysis.

Design: We report herein the microarray-based DNA methylation study of 1,505 CpG sites of cancer-related 807 genes from 69 Ewing's sarcoma samples. We used Illumina'sGoldenGate Methylation Cancer Panel I microarray.Using appropriate controls $(n=14)$, we identified a total of 92 hypermethylatedgenes in the Ewing's sarcoma samples.

Results: Most of the hypermethylated genes were related to cell adhesion, cell regulation, development and signal transduction. We compared overall methylation meansbetween patients who survived and those who did not. The overall methylation mean was significantly higher in patients who did not survive $(0.25 \pm 0.03)$ than in those who did $(0.22 \pm 0.05)(p=0.0322)$. However, the overall methylation meandid not significantly correlate with age, sexand tumor location. GDF10, OSM, APC and HOXA11 were the most significant differentially methylated genes but their methylation levelsdid not significantly correlate with the survival rate.

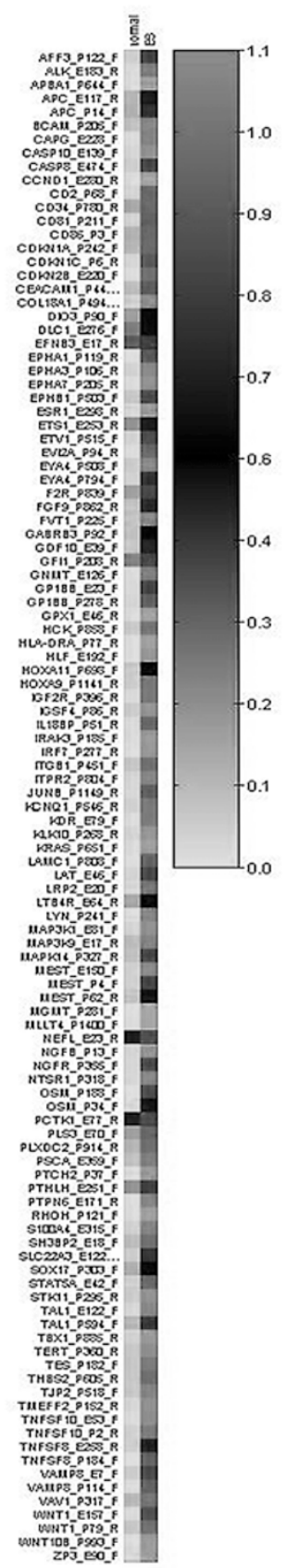

Conclusions: We characterized the DNA methylation profile of Ewing's sarcomas and detected 92 genes that were significantly hypermethylated. A trend toward more aggressive behavior was identified in the methylated group. The results of this study suggest that methylation may play an important role in the development of Ewing's sarcomas.

85 CXCL16 and CXCR6 in Ewing Sarcoma Family Tumor

B Noh, H-S Kim, W-W Jung, J-Y Sung, RK Kalil, YW Kim, Y-K Park. Graduate School of Medicine, Kyung Hee University, Seoul, Republic of Korea; College of Health Science, Korea University, Seoul, Republic of Korea; College of Health Science, Cheongju University, Chungbuk, Republic of Korea; Kyung Hee University Hospital, Kyung Hee University, Seoul, Republic of Korea; Sarah Network of Rehabilitation Hospitals, Brasilia, Brazil.

Background: Chemokines are a family of peptide mediators that play an essential role in cellular migration and intracellular communication in tumor cells as well as immune cells. We hypothesized that the CXCL16-CXCR6 ligand-receptor system plays an important role in Ewing sarcoma family tumor (ESFT) progression.

Design: Using real time quantitative reverse transcription-polymerase chain reaction, we investigated the mRNA expression of CXCL16, CXCR6 and ADAM10 in various cell lines. We also investigated the expression of CXCL16, CXCR6, ADAM10 and ADAM17 in tissue samples from 61 ESFT patients using immunohistochemistry.

Results: The mRNA expression levels of CXCL16 and CXCR6 in the ES cell line were higher than those in the other cell lines. 
Gene expression analysis

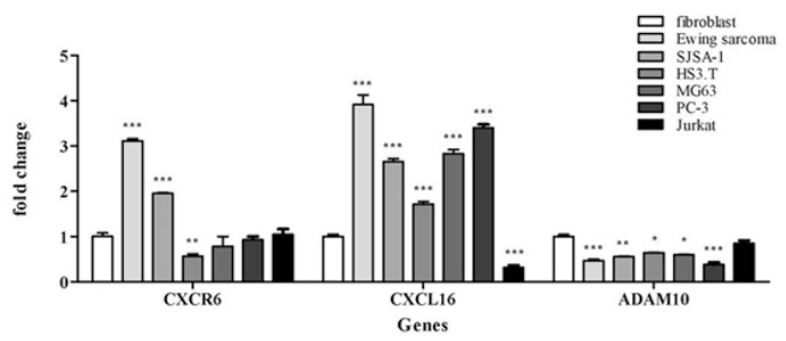

Immunohistochemical staining revealed that CXCL16 and CXCR6 were highly expressed in tumor cells of ESFT and showed a positive correlation between them. The expression of CXCL16 and CXCR6 was associated with the occurrence of lung metastasis. Univariate analysis revealed that CXCL16 or CXCR6 expression was associated with worse prognosis of ESFT patients.
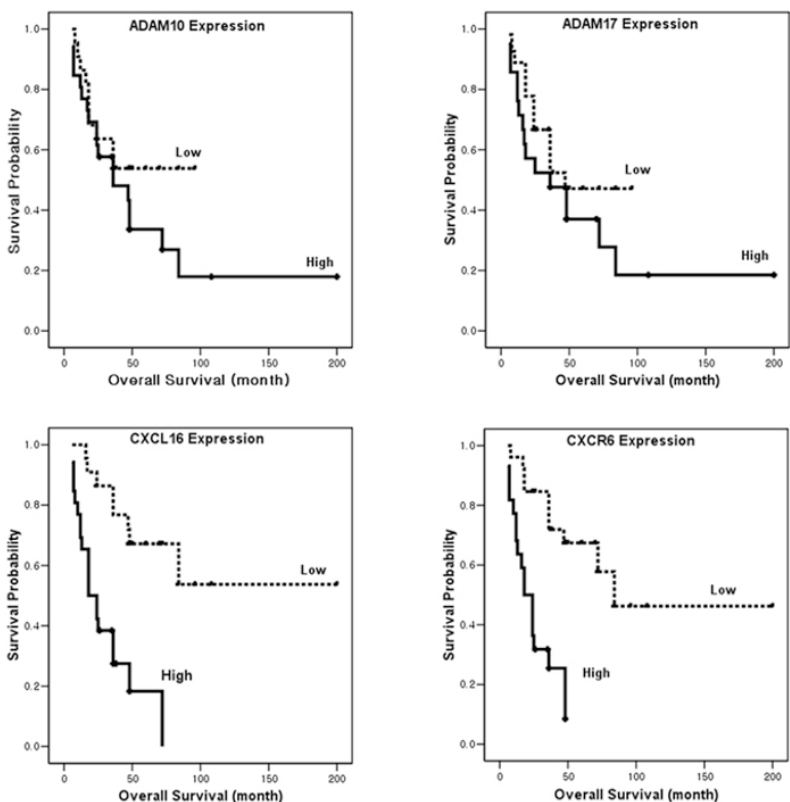

In addition, CXCL16 and CXCR6 expression was associated with shorter overall survival irrespective of other prognostic factors.

Conclusions: Our results suggest that CXCL16/CXCR6 axis appears to be important in the progression of ESFT, resulting in more aggressive clinical behavior. Furthermore, there may be a decrease in the overall survival in ESFT patients who have tumors that stain strongly for CXCL16 and CXCR6.

86 Immunohistochemical Analysis of HMGA2 Expression Fails to Provide Evidence of a Neoplastic Basis for "Primary" Synovial Lipomatosis DR Olson, JT Schowinsky. University of Colorado Denver School of Medicine, Aurora, CO.

Background: Synovial lipomatosis is characterized histologically by villous proliferation of the synovium with expansion and often replacement by mature adipocytes, occasional mild to moderate chronic inflammation, and variable synovial hyperplasia and fibrosis. Clinicopathologically, synovial lipomatosis is divided into primary disease, also known as lipoma arborescens, where the lipomatosis is the predominant pathologic feature in the joint, and secondary disease, where the lipomatosis is deemed to arise as a reaction to pre-existent joint disease, typically osteoarthritis. While some have proposed primary synovial lipomatosis to be a non-neoplastic reactive condition, it has traditionally been viewed as a variant lipomatous neoplasm, and gross and microscopic pictures of synovial lipomatosis are present in the lipoma chapter of the most recent WHO Classification of Tumours of Soft Tissue and Bone. Overexpression of HMGA2 is known to be detectable by immunohistochemistry in up to $86 \%$ of conventional lipomas, but to our knowledge has not yet been explored in synovial lipomatosis.

Design: The available surgical pathology archives of our institution (spanning 10 years) were searched for cases with the words "synovium" or "synovial" and the words "lipoma" or "lipomatosis," as well as for the phrase "lipoma arborescens." All returned cases were reviewed, and all cases clinically and histologically consistent with primary synovial lipomatosis $(\mathrm{n}=5)$ or with features indeterminate between primary and secondary synovial lipomatosis $(\mathrm{n}=3)$ were included in the study. In addition, 9 cases most consistent with secondary synovial lipomatosis (most reflecting incidental histologic findings on review of routine joint replacement specimens) were included, as well as 4 cases of conventional lipoma, and 4 samples of benign adipose tissue. Selected cases underwent immunohistochemical evaluation with a monoclonal antiHMGA2 antibody.
Results: All cases of synovial lipomatosis, regardless of classification as primary, indeterminate, or secondary, were negative for overexpression of HMGA2. 3 of 4 conventional lipomas were positive for overexpression of HMGA2, and all samples of benign adipose tissue were negative.

Conclusions: The findings support the growing hypothesis that synovial lipomatosis, including "primary" cases where the lipomatosis is the predominant pathologic finding in the joint, is a reactive non-neoplastic process.

87 Molecular Characterization of Epithelioid Hemangioendotheliomas Identifies Novel Variants of WWTR1/CAMTA1 Fusion Transcripts

NR Patel, AA Salim, H Sayeed, SF Sarabia, M Warren, J Jakacky, M Tanas, BP Rubin, AJ Lazar, D Lopez-Terrada, W-L Wang. Texas Children's Hospital/Baylor College of Medicine, Houston, TX; The University of Texas MD Anderson Cancer Center, Houston, TX; Cleveland Clinic, Cleveland, $\mathrm{OH}$.

Background: Epithelioid Hemangioendothelioma (EHE) is a lower-grade malignant vascular neoplasm that may be difficult to diagnose due to morphologic features reminiscent of both epithelial and mesenchymal tumors. Recently, two subsets have been identified by translocations resulting in the WWTR1/CAMTA1 and YAP1/TFE3 fusion genes. We sought to develop molecular and immunohistochemical (IHC) assays to aid in the diagnosis and molecular characterization of these tumors.

Design: 62 formalin-fixed, paraffin-embedded (FFPE) cases of EHE diagnosed between 2001 and 2013 were retrieved from the pathology files of our institutions. Ages at presentation ranged from 12 to 78 (median: 46) years, with a male: female ratio of 33:29. Locations included: liver, lung, pericardium, skull, and long bones. Multiple primer sets were designed to optimize a RT-PCR assay for the detection of WWTR1/ CAMTA1 fusion transcripts in FFPE tissue. In order to screen for the YAP1/TFE3 fusion gene, TFE3 protein overexpression was examined by IHC.

Results: RNA was extracted from 32 cases, with more recent cases providing a greater yield of high quality RNA. 8 of $10(80 \%)$ informative cases were positive for WWTR1/CAMTA1 fusion transcripts by RT-PCR, one of which showed malignant features. Previously undescribed in-frame fusion transcripts were identified in 3 cases by Sanger sequencing. In one case, new fusion sites within exon 3 of $W W T R I$ and exon 9 of CAMTA 1 were identified. Two cases demonstrated fusion of WWTR 1 exon 2 and portions of intron 2 to new sites within exon 9 of CAMTA1. IHC revealed variable nuclear staining for TFE3 in 9 of 24 cases; three with patchy staining were found to have the WWTR1/CAMTA1 fusion transcript.

Conclusions: RT-PCR for WWTR1/CAMTA1 fusion transcript detection can be applied to clinical FFPE specimens to support the diagnosis of EHE; and fusion transcripts can be identified in cases with malignant features. We demonstrate additional structural complexity in WWTR1/CAMTA1 fusion transcripts. Initial results suggest that IHC for TFE3 is not entirely specific and further molecular testing is recommended. Molecular testing of additional cases, including identification of YAP1/TFE3 fusion, is currently on-going.

$88 \quad$ CIC-Rearranged Pediatric Sarcomas Associated with Novel Translocations and Unusual Clinical Presentations

NR Patel, D Sugganth, EF Hollingsworth, D Tran, V Mehta, T Peters, BP Rubin, J Hicks, $R$ Angshumoy, D Lopez-Terrada. Texas Children's Hospital/Baylor College of Medicine, Houston, TX; Cleveland Clinic, Cleveland, OH.

Background: The accurate classification of pediatric primitive sarcomas has remained challenging. Some of these cases have recently been shown to harbor recurrent translocations involving the $C I C$ gene, such as $\mathrm{t}(4 ; 19)$ and $\mathrm{t}(10 ; 19)$. In this study, we sought to identify additional pediatric cases to characterize their genetic alterations, as well as their histopathology and clinical characteristics.

Design: We searched the pathology files of our institution for primitive sarcomas confirmed to be negative for sarcoma-associated translocations. 26 cases diagnosed from 2001 to 2012 were identified and 8 unique patient samples with a small blue round cell morphology were retrieved. FISH analysis was performed using a dualcolor interphase break-apart probe for the 5' and 3' regions of the CIC gene. Complete karyotypes were available in 6 cases.

Results: 5 of 7 cases showed $C I C$-rearrangements by FISH; one case could not be interpreted due to poor preservation. Patients with $C I C$-rearranged tumors showed a male: female ratio of $4: 1$ and mean age of 14.8 years at diagnosis. Primary lesions involved the liver, right hemithorax, pelvic soft tissue, and thigh, measuring from 3.5 to $28.9 \mathrm{~cm}$. 3 cases showed multifocal disease at presentation. Histologically, they consisted of sheets of primitive round cells with occasional prominent nucleoli, focal spindle cells, geographic necrosis, and brisk mitotic activity. Immunohistochemical staining for CD99 was variably positive in a cytoplasmic or membranous pattern. One patient presented with a large liver mass encasing the colon and a diaphragmatic implant, and had a complex tumor karyotype with $\mathrm{t}(\mathrm{X} ; 19),+8,+12,+20$; this patient is free of disease 3 years post-resection, chemotherapy and radiation. A second patient presenting with multifocal involvement of the scrotum and inguinal soft tissue - and a tumor carrying a $t(4 ; 19 ; 6),+8$ with a subclone harboring a $t(X ; 5)$ translocation - developed recurrence post-chemotherapy and resection with subsequent death. A third patient presenting with a $28.9 \mathrm{~cm}$ thigh mass and metastases to the lung and lymph nodes also showed chemoresistance and died despite therapy.

Conclusions: $C I C$-rearranged Primitive Sarcomas are clinically aggressive and, thus, critical to recognize. We identified additional pediatric cases showing a wide clinical spectrum and novel cytogenetic findings associated with the $C I C$ rearrangement, in addition to trisomy 8 . Molecular studies are currently being performed to further characterize the variant translocations and fusion transcripts. 


\section{The BCOR/CCNB3 Fusion Gene Is Present in a Subset of} Undifferentiated Pediatric Sarcomas

TL Peters, VKumar, D Lopez-Terrada, A Roy. Texas Children's Hospital, Houston, TX; Baylor College of Medicine, Houston, TX.

Background: The BCOR/CCNB3 fusion gene, resulting from a chromosome $\mathrm{X}$ paracentric inversion, was recently described in $4 \%$ of bone sarcomas with "Ewing's-like" features. Here, we investigated the prevalence of this fusion gene in undifferentiated/unclassified childhood sarcomas, a group of tumors lacking defining morphological features or markers.

Design: An institutional database was searched over a 10-year period to retrieve archived cases of undifferentiated sarcoma, small round cell tumor, peripheral primitive neuroectodermal tumor and/or Ewing sarcoma-like tumor, excluding cases with characteristic fusion genes or translocations detected by RT-PCR, FISH and/or cytogenetics. Three Ewing sarcomas with documented EWSR1 rearrangement served as negative controls. A RT-PCR assay was designed to amplify the BCOR/CCNB3 fusion from formalin-fixed paraffin-embedded and fresh-frozen tissue, with primers on BCOR exon 15 and CCNB3 exon 5. Long-range PCR and primer walking were used to map genomic breakpoints. CCNB3 expression was evaluated using a commercially available antibody.

Results: Twelve samples ( 3 bone, 9 soft tissue) from twelve unique patients (ages 1-17 years; $\mathrm{M}: \mathrm{F}=2: 1$ ) were tested. RT-PCR on RNA from FFPE tissue revealed 3 cases with robust amplification of a 171-bp product with sequencing revealing an in-frame fusion of BCOR exon 15 and CCNB3 exon $5(3 / 12=25 \%)$ that resulted from cryptic splice site activation in BCOR and skipping of the stop codon. The genomic breakpoint in each case mapped to unique sites within CCNB3 intron 4. All three cases were soft tissue tumors in male patients demonstrating both large pleomorphic round cells and areas of spindled cells with brisk mitotic activity, expressing vimentin and variable CD99. Tumor cells had prominent nuclear expression of CCNB3. The fusion was detected in multiple recurrences and metastatic lesions in two positive patients who succumbed to disease. Conclusions: This study expands the clinical and morphological spectrum of BCOR/ CCNB3-positive sarcomas. In our series of undifferentiated/unclassified sarcomas, fusion-positive lesions were soft tissue tumors with undifferentiated round and spindle cell morphology. Molecular techniques unambiguously identified fusion junctions providing a useful aid for diagnosis. The BCOR/CCNB3 oncoprotein may drive tumorigenesis through aberrant cell cycle regulation. Further studies will shed light on the pathobiology of these tumors.

\section{Human Cytomegalovirus Is Present in Alveolar Soft Part Sarcoma} RL Price, H Kurt, L Harkins, AE Chiocca, PZ Zhang, OH Iwenofu. The Ohio State University, Columbus, $\mathrm{OH}$; Brigham and Women's Hospital and Dana-Farber Cancer Institute, Boston, MA; Hospital of the University of Pennsylvania, Philadelphia, PA. Background: Alveolar Soft Part Sarcoma (ASPS) is an exquisitely rare sarcoma of unknown histogenesis, with a predilection for adolescents and young adults, characterized by slow progressive clinical course and high frequency of metastases. These tumors are often chemoresistant, thus surgery remains the mainstay of therapy with very limited treatment options in the metastatic setting. Human cytomegalovirus (HCMV) is a DNA $\beta$-herpes virus and like all herpes viruses, it is characterized by persistent lifelong and latent infection. There is growing evidence to indicate the presence of HCMV proteins and nucleic acids in glioblastoma multiforme (GBM), medulloblastoma and a variety of solid organ malignancies of the breast, prostate, lung and colon at very high prevalence. Immunotherapy based clinical trials targeting specific CMV proteins are currently in progress in the treatment of GBM. Herein, we evaluated for the presence of HCMV proteins (IE1 and pp65), genes (US28 and UL96) and RNA by immunohistochemistry (IHC), DNA polymerase chain reaction (PCR), and in situ hybridization respectively in a cohort of ASPS.

Design: 6 confirmed cases of ASPS were retrieved from the archives of the Departments of Pathology at OSUMC and the University of Pennsylvania. Full thickness sections of formalin fixed paraffin embedded material were stained for anti-HMCV-IE1 and anti-HCMV-pp65. Any nuclear and/or cytoplasmic staining was considered positive. DNA was purified from $50 \mu$ of FFPE material. One hundred ng of DNA was amplified using PCR for primers specific to HCMV-US28 (forward: AGCGTGCCGTGTACGTTAC, and reverse: ATAAAGACAAGCACGACC) and HCMV-UL96 (forward: ACAGCTCTTAAAGGACGTGATGCG, reverse: ACCGTGTCCTTCAGCTCGGTTAAA) using Promega (Madison, WI) Taq polymerase according to manufacturer's protocol. HCMV in situ hybridization (ISH) was performed. Results: All 6 cases of ASPS were positive for both HCMV-IE1 and HCMV-pp65 by IHC. Usable DNA was available in 4 of the 6 cases. DNA from the HCMV-US28 gene was found in $75 \%(3 / 4)$ of cases and HCMV-UL96 gene was detected in 50\% (2/4) of cases. Importantly, all cases tested positive for at least one gene. 3/4 cases were positive for HCMV by ISH.

Conclusions: This is the first study to detect HCMV in ASPS. The presence of HCMV DNA, RNA along with HCMV protein indicates that HCMV is present in ASPS and may contribute to it's pathogenesis and provide a basis for targeted immunotherapy.

91

BCOR-CCNB3 Sarcoma: A Single Institution Experience of 8

Cases

F Puls, A Niblett, G Marland, V Sumathi, L-G Kindblom. Royal Orthopaedic Hospital, Birmingham, United Kingdom.

Background: BCOR-CCNB3 fusion transcripts were recently identified in a series of undifferentiated small cell sarcomas with Ewing's-like features. BCOR-CCNB3 sarcoma appears to be rare: to date only a single case series has been published. To further characterize its morphological features and clinical course as well as diagnostic tools for its recognition, we report our experience of 8 cases of BCOR-CCNB3 sarcoma.
Design: Undifferentiated small cell sarcomas without Ewing's sarcoma associated transcripts and monotonous morphology with small to medium cell size were immunohistochemically stained for CCNB3. RNA was extracted from formalin-fixed paraffin-embedded and freshly frozen tumor material if available. BCOR-CCNB3 fusion transcripts were identified by RT-PCR and confirmed by Sanger sequencing. Clinical follow up was obtained.

Results: 8 sarcomas that harbor BCOR-CCNB3 transcripts were identified among 92 cases of undifferentiated sarcomas with no or unknown transcripts. Immunohistochemistry showed nuclear positivity in all BCOR-CCNB3 cases, but also showed non-specific staining in cytoplasm and in necrotic areas of BCOR-CCNB3 negative sarcomas. On mRNA level all cases showed identical break-points. All cases showed discohesive small, plump to slightly spindle cell morphology with angulated nuclei lying within an edematous matrix. 7 of 8 patients were male. The age range was 13 to 18 years). 5 tumors were primarily located in bone and 3 in deep soft tissues. 3 occurred in the axial skeleton or soft tissues, 5 in the appendicular skeleton. 3 patients had metastatic disease at the time of diagnosis (lung x2, osseous x1). All patients were treated according to Ewing's sarcoma protocols. 3 patients died of disease 27, 28 and 110 months after diagnosis, all of whom had tumors located in the axial skeleton, not amenable to curative resection. 3 patients are alive without evidence of disease at 69 , 116 and 124 months. 2 patients are currently still receiving treatment.

Conclusions: BCOR-CCNB3 sarcomas are rare primitive small cell malignancies occurring in bone as well as in soft tissues. There is a male preponderance and the peak incidence is in the 2 nd decade. Immunohistochemistry is a useful tool for screening, however RT-PCR and sequencing should be used for definite identification. Correct identification of BCOR-CCNB3 sarcoma appears important for further characterization of this rare sarcoma.

92 Histopathological, Immunohistochemical and Molecular Cytogenetic Analysis of 22 Spindle Cell/Sclerosing Rhabdomysarcomas B Rekhi, T Singhvi. Tata Memorial Hospital, Mumbai, Maharashtra, India.

Background: As per the current WHO classification of soft tissue tumors, spindle cell and sclerosing rhabdomyosarcoma(RMS), together, have been recognized as another variant of RMS.

Design: We evaluated clinicopathological, immunohistochemical(IHC) and molecular cytogenetic features of 22 spindle cell/sclerosing RMSs. Eleven tumors were tested for FISH analysis with RMSI, SPEC $t(2 ; 13)$ and RMS II, SPEC $t(1,13)$ dual color fusion probes.

Results: Twenty-two tumors in 16 males and 6 females(M: F, 2.6:1), with age ranging from 2-60 years(Mean,21) and size(14 cases), varying from 3-15 $\mathrm{cm}$ (Average, 7.9) occurred in head and neck(8)(36.3\%), extremities $(8)(36.3 \%)$,paratesticular region(2) (9\%),chest wall(1), abdomen(1),pelvis(1) and paraspinal region(1). Histopathologically, tumors were sclerosing (10)(45.4\%), mostly in extremities; spindle cell (8) (36.3\%),mostly in head and neck and sclerosing with spindle cell types (4)(18.1\%). Tumor cells, including rhabdomyoblasts(7 tumors) were variably arranged, especially sclerosing RMSs in pseudovascular, focal embryonal( 5 cases) and alveolar( 3 cases) patterns; with their cells embedded in hyaline and/ or pseudochondroid matrix. Overall, immunohistochemically, tumor cells were positive for desmin $(22 / 22)(100 \%)$, MyoD1(mostly diffuse)(19/19)(100\%), myogenin(focal)(14/16)(87.5\%), SMA(mostly weak)(2/3) and MIC2(weakly cytoplasmic)(2/8)(25\%). On FISH analysis, only a single tumor exhibited RMS1 fusion.

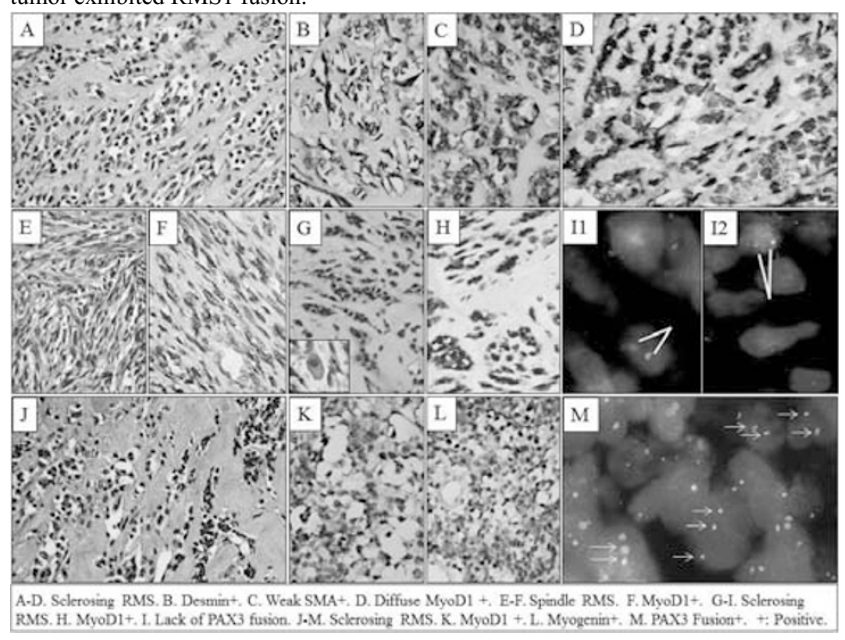

Nineteen patients underwent surgical resection and were offered adjuvant chemotherapy(CT)( 5 cases), adjuvant radiotherapy( 1 case $)$ and adjuvant $\mathrm{CT}+\mathrm{RT}(4$ cases); 2 patients underwentCT and 1 patient underwentCT+RT. On follow-up(17 cases)(2-36 months), 6 tumors recurred and 10 metastasized, mostly to lungs. Finally, 13 patients were alive-with-disease(2-36 months), 3 were free-of-disease(6-12 months) and a single patient died-of-disease( 16 months).

Conclusions: Spindle/sclerosing RMSs are aggressive tumors with proclivity for head and neck and extremities. Spindle and sclerosing RMSs are histopathologically related, considering certain tumors exhibit both patterns. IHC and molecular cytogenetic profile(to an extent) of these tumors are similar to embryonal RMSs. Rare cases can show histopathological and genetic features similar to alveolar RMS. 


\section{Angiosarcoma}

W Rizzo, RI Steven, B Darya, F Karen. Mayo Clinic, Rochester, MN; University of Wisconsin, Madison, WI.

Background: Angiosarcoma may present a diagnostic challenge in poorly differentiated cases. Although several markers of endothelial differentiation are available, their sensitivities vary, and it is not uncommon to encounter an angiosarcoma which is negative for one or more of these. Additionally the therapeutic options for angiosarcoma are limited. Endoglin/CD105 is a homodimeric transmembrane glycoprotein expressed on the surface of endothelial cells, and there is evidence that anti-CD105 antibodies bind preferentially to activated endothelial cells. In this study, we investigated the value of anti-CD105 antibodies in the diagnosis of angiosarcoma. We also explored the expression of CD105 in non-neoplastic blood vessels in tumor and peri-tumoral tissue in angiosarcoma to evaluate CD105 as a potential therapeutic target.

Design: Immunohistochemistry for CD105 (Leica Biosystems) for was performed on 146 sarcomas and 12 benign vascular proliferations from the Mayo Clinic and University of Wisconsin tumor databases including 20 angiosarcomas, 19 chondrosarcomas, 7 Ewing sarcomas, 20 osteosarcomas, 40 leiomyosarcomas (LMS) (20 gynecologic, 20 non-gynecologic), 20 synovial sarcomas, 20 undifferentiated pleomorphic sarcomas and 12 hemangiomas. Following verification of diagnosis, either cytoplasmic or membranous expression was scored semi-quantitatively as $0(<5 \%$ cells staining $), 1+(5-24 \%), 2+$ $(25-49 \%)$, and $3+(\geq 50 \%)$. Tumors with scores of $2+$ and $3+$ were considered positive. Intra-tumoral and peri-tumoral non-neoplastic blood vessels in cases of angiosarcomas were qualitatively evaluated as positive or negative.

Results: $80 \%$ of angiosarcomas $(16 / 20)$ and $67 \%$ of benign vascular proliferations (8/12) were positive for CD105, while the vast majority of nonvascular sarcomas $(113 / 126)$ were negative. No chondrosarcomas $(0 / 19)$ or synovial sarcomas $(0 / 20)$ expressed CD105, while one Ewing sarcoma (1/7), 2 osteosarcomas (2/20), 5 undifferentiated pleomorphic sarcomas (5/20), 2 non-gynecologic LMS (2/20), and 3 gynecologic LMS (3/20) were positive. In cases of angiosarcoma which had evaluable non-neoplastic vasculature, CD105 was positive in all intra-tumoral (17/17) and peri-tumoral (16/16) vessels.

Conclusions: CD105 is a sensitive ( $80 \%)$ and specific $(90 \%)$ marker for angiosarcoma and may be helpful in poorly differentiated cases to confirm lineage. CD105 is a potential therapeutic target as it is expressed in both lesional cells and associated non-neoplastic vessels.

\section{4}

\section{Analysis}

SRossi, M Cacciatore, D Gasparotto, L Toffolatti, V Carraro, G Gallina, E Scaramel, E Boscato, M Niero, I Bearzi, A Mandolesi, F Sessa, A Sonzogni, C Mancini, P Amore, $R$ Maestro, AP Dei Tos. General Hospital, Treviso, Italy; CRO, Aviano, Italy; University of Marche, Ancona, Italy; Macchi Fondation, Varese, Italy; General Hospital, Bergamo, Italy; Parma University, Parma, Italy; Novartis Farma, Origgio, Italy.

Background: V600E BRAF mutation occurs very rarely in GISTs $(5-20 \%$ of KIT/ PDGFRA wild type GISTs) and acts as a mechanism alternative to KIT/PDGFRA mutation. The performance of BRAF-V600E specific antibody (VE1) has been analyzed on a variety of tumors, with heterogeneous results. Our goal was to test the utility of this antibody in GISTs.

Design: A first set of 25 GISTs on freshly cut whole section with 5 BRAF-mutated and 20 BRAF WT cases (16 KIT and 4 PDGFRA-mutated) and a second set of 230 GISTs on tissue microarray (TMA), including 2 BRAF-mutated cases and 228 control cases (152 KIT, 52 PDGFRA mutated and 24 KIT/PDGFRA/BRAF WT cases) were immunostained (clone VE1, Spring Bioscience, Pleasanton, CA) by an automated immunostainer (Dako Autostainer, DakoCytomation, Glostrup, Denmark). In order to optimize the method, a range of conditions were tested ( $\mathrm{pH} 6$ and $\mathrm{pH} 9$ buffer; 1:25, 1:50, 1:100 dilutions) and $\mathrm{pH} 9$ and 1:100 were used. Cases without staining or with a very faint staining barely perceptible at high power were considered as negative. Cases with a cytoplasmic staining were considered positive and scored as weak, moderate and strong. Results: Of the first set, the 5 BRAF-mutated GISTs were VE1 positive ( 2 weak, 1 moderate, 2 strong), with a diffuse staining in 4 cases and focal in 1 . The 20 BRAF-WT cases were negative. Of the second set, the $2 \mathrm{BRAF}$-mutated cases were VE1 positive (1 weak and 1 moderate).and, among the control cases, 209 (91,7\%) were negative, whereas $19(8,3 \%)$ cases were weakly positive. In the latter subset KIT/PDGFRA-mutated VE1 positive cases, co-existence of KIT/PDGFRA and V600E BRAF mutation was ruled out by subsequent BRAF sequencing.

\begin{tabular}{|l|l|l|l|l|l||l||}
\hline \hline & VE1 & & \\
\hline \hline & Lhole section & TMA & Total & \\
\hline \hline & neg & pos $(1+/ 2+/ 3+)$ & neg & pos $(1+/ 2+/ 3+)$ & & \\
\hline $\begin{array}{l}\text { V600E mutated } \\
\text { GISTs }\end{array}$ & 0 & $5(2 / 1 / 2)$ & 0 & $2(1 / 1 / 0)$ & 7 & sensitivity $7 / 7(100 \%)$ \\
\hline Control GISTs & 20 & 0 & 209 & $19(19 / 0 / 0)$ & 248 & Specificity 229/248 (92,3\%) \\
\hline \hline & & & & & 255 & concordance 236/255(92,5\%) \\
\hline
\end{tabular}

Conclusions: Overall the concordance rate between immunohistochemistry and molecular analysis was $92,5 \%$, with VE1 sensitivy of $100 \%$ and specificity of $92,3 \%$. Notably, 3/7 BRAF-mutated GISTs showed a weak VE1 staining as well as a subgroup of control GISTs. Thus, in order to confirm the presence of V600E mutation, GISTs with weak VE1 expression should be sequenced.
95 Aggressive Angiomyxoma (AAM) of Men and Women: Clinicopathologic Review of 20 Cases

AA Salim, D Stockman, J Meis. MD Anderson Cancer Center, Houston, TX.

Background: Aggressive angiomyxomas are rare, locally aggressive, mesenchymal fibromyxoid tumors involving the vulvovaginal, pelvic and perineal regions occurring primarily in women. Aggressiveness in these tumors stems from their diffusely infiltrative nature and difficulty in surgical extirpation due to the intricacy of involved anatomic structures. Clinical misdiagnoses as Bartholin or vaginal cysts, lipomas and hernias and a paucicellular banal appearance in core biopsy specimens frequently lead to delayed diagnoses.

Design: Twenty patients with AAM were referred to our center over the last 27 years (1986 - 2012). Clinical data and specimens, including biopsies and resections, were reviewed.

Results: Tumors occurred in 3 men and 17 women with a median age of 41 years (range, 20 to 86). The tumors ranged from 5.3 to $30 \mathrm{~cm}$ (median $12.7 \mathrm{~cm}$ ) in size, and were distributed as follows: vulva in $2 / 20$ cases $(5 \%)$, pelvis in $13 / 20 \operatorname{cases}(31 \%)$, vagina in $1 / 20$ cases $(2 \%)$, perineum in $3 / 20$ cases $(7 \%)$, and retroperitoneum in $1 / 20$ $(2 \%)$. Histologically, the tumors were comprised of infiltrative, sparsely populated bland-looking spindled and stellate cells in a fibromyxoid matrix with admixed small and large blood vessels. By immunohistochemistry, tumors were positive for ER and PR (11) and desmin (9). Clinical follow-up was available in 15 patients with median follow-up of 20 mo (range, 2-307 mo). There were recurrences in 6/20 cases (30\%); median interval to recurrence was 13 months (range 5-307 mos).

Conclusions: Aggressive angiomyxoma is a potentially aggressive, non-metastasizing soft tissue tumor occurring in both men and women. Definitive diagnosis in core biopsy specimens is particularly difficult when fatty infiltration is not present, small vessels are not numerous, or entrapped smooth muscle is seen. Large caliber vessels are often prominent in resection specimens as are less myxoid zones that may overlap or cause confusion with angiomyofibroblastoma. Cases occurring in males have a less prominent fatty component.

\section{Clinical Next Generation Sequencing of Sarcomas: Initial} Experience of 133 Cases

AA Salim, W-L Wang, K Aldape, R Luthra, M Routbort, A Lazar. MD Anderson Cancer Center, Houston, TX.

Background: Clinical next generation sequencing (NGS) allows for efficient and simultaneous mutational assessment of multiple genes. The results can have important implications in terms of therapeutic selection. Bone and soft tissue sarcomas are uncommon and other than certain gene fusions, relative little is known of the mutations that drive their genomes and malignant states. Since April 2012, clinical NGS has been performed on more than 3,500 tumors at our institution. Here we describe our initial experience with the application of a targeted clinical NGS panel on a variety of sarcoma cases

Design: Our molecular diagnostics lab database of clinical NGS was searched for soft tissue and bone sarcomas and 133 cases were recovered. During the time of this study (4/2012-9/2013) our clinical NGS panel (Ampliseq, Life Technologies) consisted of the commonly mutated regions of 46 genes (focused on clinically actionable or common mutations in carcinoma, melanoma and brain tumors). The results were tabulated to determine mutation prevalence in sarcomas.

Results: Out of these 133 tumors, 20 (15\%) had TP53 mutations, $3(2 \%)$ had $I D H I$ mutations, $3(2 \%)$ had $B R A F$ mutations, $2(1 \%)$ had ATM mutations, and $2(1 \%)$ had $A P C$ mutations. Eight genes showed mutations in one case each (RB1, PTEN, NRAS, KRAS, GNAS, FGFR1, ERBB4 and CDKN2A), while 102 cases (76\%) showed no somatic mutations by this targeted panel. Mutational prevalences varied amongst sarcomas types. For instance, TP53 mutations were very rare in Ewing sarcoma/PNET ( 2 of 29 cases), but relatively common in unclassified sarcomas ( 9 of 34 cases). Of note, five tumors showed more than one mutation: chondrosarcoma ( 1 case), dedifferentiated chondrosarcoma (1), rhabdomyosarcoma (1) and unclassified sarcoma (2).

Conclusions: Our study shows that mutation rates are relatively low amongst sarcomas. However, this may be due to the use of a panel optimized for mutational events in non-mesenchymal tumors. As the genomic features of sarcomas are better defined, it is anticipated that NGS panels more appropriate to sarcomas can be developed.

\section{Myxoid Variant of So-Called "Angiomatoid MFH"}

I-M Schaefer, CDM Fletcher. Brigham and Women's Hospital and Harvard Medical School, Boston, MA.

Background: Angiomatoid malignant fibrous histiocytoma (AMFH) is a tumor of intermediate malignancy and undefined lineage, mostly arising in the extremities of young patients. Examples with a prominent myxoid matrix are very uncommon. Although these lesions seem to show similar epidemiologic, clinical, and molecular characteristics as conventional AMFH, predominant myxoid features may complicate the diagnosis.

Design: Twenty cases of myxoid AMFH (among a total of 414) were identified in consult files between 1992 and 2013. H\&E and immunohistochemical stains were examined and FISH analysis was performed in a subset of cases. Clinical and follow-up information was obtained from referring pathologists.

Results: Twelve patients were female and 8 male, aged between 2 and 51 years (median, 16). The tumors were mostly circumscribed and arose in subcutaneous or deep soft tissue with tumor size ranging from 1 to $8 \mathrm{~cm}$ (median, 2.5). The majority of tumors arose in the extremities (13/20), followed by trunk (4/20) and limb girdles $(3 / 20)$. Characteristic features included a fibrous pseudocapsule (19/20), prominent peritumoral lymphoplasmacytic infiltrates (19/20), and blood-filled cystic spaces (16/20). All tumors showed prominent areas with myxoid morphology, comprising 60 to $100 \%$ of 
the entire tumor. Histiocytoid tumor cells exhibited oval or polygonal vesicular nuclei, inconspicuous nucleoli, and palely eosinophilic cytoplasm with ill-defined borders. Typical multinodular growth was observed with whorls or loose trabeculae of tumor cells in hypocellular areas with prominent myxoid matrix and occasional transition to more solid nodules. Mucin pools and scattered multinucleate giant cells were observed in a subset of cases. Mitoses were scant and no necrosis was present. Mild to moderate atypia was observed in 4 cases; 1 tumor showed rhabdoid features. Tumor cells expressed EMA (11/20), desmin (13/20), CD99 (2/3), and CD68 (2/6). FISH was performed in 7 tumors and revealed $E W S R I$ rearrangement in 4 cases. Five tumors were excised with a suspected diagnosis of sarcoma; 1 patient received preoperative radiotherapy. Followup data, available for 9 cases (median, 40 months), revealed that 3 patients developed local recurrence after 2, 7, and 48 months, respectively. All patients were alive without evidence of disease and none have developed metastases.

Conclusions: AMFH may rarely present with a prominent myxoid matrix, making diagnosis more difficult and causing possible confusion with other myxoid tumors such as extraskeletal myxoid chondrosarcoma, myoepithelial tumors, and low-grade fibromyxoid sarcoma.

98 Validation of Break Apart FISH Probes for the Detection of COL1A1PDGFB Rearrangements in Dermatofibrosarcoma Protuberans

$H-U$ Schildhaus, E Binot, R Buttner, I Tancheva-Poor, E Wardelmann. University Hospital Cologne, Cologne, Germany; University Hospital Göttingen, Göttingen, Germany; University Hospital Münster, Münster, Germany.

Background: Dermatofibrosarcoma protuberans (DFSP) is a malignant mesenchymal tumor of the skin. The underlying molecular mechanisms are various chromosomal rearrangements which result in the formation of a COL1A1-PDGFB fusion gene. Clinically progressive DFSP with these genetic alterations respond to treatment with the tyrosine kinase inhibitor Imatinib. Therefore, the detection of that gene fusion has become one of the rare predictive biomarkers in sarcomas. Traditionally, the COL1A1PDGFB fusion is determined by RT-PCR or so-called dual color dual fusion FISH probes which cover both involved genes. The aim of our study was to compare different FISH approaches and to validate the most suitable one.

Design: A total of fifty tumors (19 DFSP as well as 21 dermatofibromas and cellular/ deep penetrating dermatofibromas) were investigated by using specific COL1A1-DFSP dual color dual fusion, COL1A1-PDGFB dual color single fusion, PDGFB break apart and COL1A1 break apart FISH probes. The percentages of aberrant tumor cells were determined for each lesion and each probe set.

Results: Two out of eighteen evaluable DFSP (11\%) were false negative with the dual color dual fusion probes (but clearly positive for all of the other three probes), indicating that the sensitivity of these probes is comparably low. The dual color single fusion probe approach revealed positive results in 100\% of DFSP. However, 14/16 $(88 \%)$ of all evaluable dermatofibromas revealed $>15 \%$ aberrant tumor cells with those probes indicating a high proportion of false positive cases. Best results were achieved with the break apart probes for both genes which resulted in $100 \%$ sensitivity and specificity. Therefore, cut-off levels were determined for the break apart probes statistically (based on the percentages of randomly "aberrant" signals determined in the dermatofibroma cohort)

Conclusions: We could demonstrate that the widely used dual color dual fusion probes do not recognize all types of rearrangements and that nearly $10 \%$ of cases will be missed by that approach. Dual color single fusion probes, on the other hand, are often false positive and may result in a misdiagnosis of DFSP, especially in cases of cellular/deep penetrating dermatofibromas. $\mathrm{PDGFB} / \mathrm{COL} 1 \mathrm{~A} 1$ break apart probes are reliable techniques for the detection of therapeutically relevant COL1A1-PDGFB fusions in DFSP.

\section{$99 \quad$ Primary Pulmonary Myxoid Sarcoma Versus Pulmonary} Angiomatoid Fibrous Histiocytoma: Cases with Overlapping Features?

L Schmidt, SC Smith, N Palanisamy, B Betz, SA Tomlins, R Mehra, DR Lucas, JL Myers. University of Michigan, Ann Arbor, MI

Background: Primary pulmonary myxoid sarcoma (PPMS) is a recently described, potentially malignant lesion showing polygonal and spindled to stellate cells within myxoid stroma. Reported examples show endobronchial growth and a EWSR1-CREB1 fusion in $\sim 75 \%$ of documented cases. Recent appreciation of an expanded anatomic and morphologic spectrum of angiomatoid fibrous histiocytoma (AFH), which shares the same translocation, raises the possibility that these two might be overlapping entities. Design: We reviewed clinicopathologic features and performed breakapart FISH assays on 3 cases from consultation services at our institution.

Results: Three cases of PPMS arose in one woman and two men (Cases A-C, see Table 1). All patients underwent lobectomy which showed grossly endobronchial- $(A, C)$ or bronchial-based (B) pale tan-gray glistening nodular to partially encapsulated masses with consolidation of distal lung. All cases showed lobulated to multinodular neoplasms comprising a prominent myxoid stroma containing single epithelioid to spindled/stellate cells arranged individually, in clusters, or in a reticular pattern. AFH-like features included at least focal peritumoral fibrous cuffs (A-C), blood-filled spaces (B), and reactivity for desmin (B). Associated inflammation was mild-moderate and included a prominent lymphplasmacytic infiltrate in each case. Atypia was prominent in one case (C). Broad-spectrum keratins were negative; focal EMA positivity was present in all cases. EWSR1 rearrangement was present in two (A, B), one of which additionally showed CREB1 rearrangement (A). No rearrangements were detected at the ATF1 locus. All three patients are free of disease.

Table 1
\begin{tabular}{|l|l|l|l||l|l||}
\hline Patient & Sex/Age & Presentation (Mo). & $\begin{array}{l}\text { Location/ } \\
\text { Size } \mathbf{( c m})\end{array}$ & FISH Result & $\begin{array}{l}\text { Status/ } \\
\text { follow-up } \\
\mathbf{( m o})\end{array}$ \\
\hline \hline A & F/64 & $\begin{array}{l}\text { Recurrent pneumonia } \\
(24)\end{array}$ & LUL/4.0 & EWSR1+, CREB1+, ATF1- & NED/18 \\
\hline B & M/28 & $\begin{array}{l}\text { Recurrent cough } \\
\text { hemoptysis (4) }\end{array}$ & RLL/8.5 & EWSR1+, CREB1-, ATF1- & NED/16 \\
\hline C & M/28 & Chest pain (<1) & RUL/6.0 & EWSR1-, CREB1-, ATF1- & NED/4 \\
\hline
\end{tabular}

Conclusions: All three cases were diagnosed as PPMS. PPMS which may show features overlapping with AFH rendering distinction difficult; future efforts should study the relationship between these lesions in larger multicenter cohorts.

100 Routine Histopathologic Examination of Hip and Femoral Head Excision: A 13-Year Review of Clinicopathologic Discrepancies in Consecutive 2505 Hip Arthroplasty Specimens at a Large Teaching Hospital

U Sheikh, M Khurram, P Kowalski. St. John Hospital \& Medical Center, Detroit, MI. Background: The value of routine histopathologic examination of hip arthroplasty specimens has long been a source of controversy between orthopedic surgeons and histopathologists. For that matter, consensus does not exist as to the appropriate handling of these specimens. The study aimed to quantify the discrepancy rate between clinical and histopathologic diagnosis, and to determine whether microscopic examination of femoral heads is indeed justified.

Design: A 13 year retrospective review of 2505 hip arthroplasty specimens was performed at a large tertiary care teaching institution. Clinical diagnosis was compared with final pathologic diagnosis for each case, and major and minor discrepancies were documented.

Results: Overall, there was a major discrepancy rate of $2 \%$ (48/2505) between the submitted clinical and final pathologic diagnosis. Among these, the highest rate of discrepancy was present in malignant diagnosis [64\% (31/48)], followed by osteomyelitis [22\% (11/48)]. The cases with major discrepancies were those patients for whom the treatment and/or prognosis was altered following histopathologic diagnosis. Major discrepancies included both clinically unsuspected histopathologic findings and clinical suspicion not confirmed by microscopic examination (See table 1). In remainder, they were considered minor, with more academic than therapeutic significance (e.g. osteoarthritis versus avascular necrosis of the femoral head).

Table 1. Major Discrepancies Between Clinical Impression And Histopathologic Findings
\begin{tabular}{|l|l|l||}
\hline \hline Diagnosis & $\begin{array}{l}\text { linically } \\
\text { unsuspected }\end{array}$ & $\begin{array}{l}\text { Clinically suspected but not } \\
\text { identified histopathologically }\end{array}$ \\
\hline Malignancy-Epithelial & 5 & 15 \\
\hline Malignancy-Hematolymphoid & 9 & 2 \\
\hline Osteomyelitis- Acute and Chronic & 11 & 0 \\
\hline Enchondroma & 2 & 0 \\
\hline Degenerative joint disease in hip dysplasia & 2 & 0 \\
\hline Pigmented villonodular synovitis & 2 & 0 \\
\hline
\end{tabular}

Conclusions: Clinical and histopathologic diagnoses of hip arthroplasty specimens were compared, yielding a relatively low overall discrepancy rate of $2 \%$ of all cases harboring major discrepancies. However, the discrepancy rate of malignant diagnosis was fairly high and noted upto $64 \%$, followed by osteomyelitis (22\%). These major diagnostic discrepancies significantly altered management and/or long-term prognosis. In our opinion, histopathologic examination of all hip arthroplasty specimens is indeed justified. Future studies to include a larger cohort are suggested to verify these results.

101 ERG Expression in Chondrogenic Bone and Soft Tissue Tumors $W$ Shon, AL Folpe, KJ Fritchie. University of Florida, Gainesville, FL; Mayo Clinic, Rochester, MN.

Background: $E R G$ gene encodes for an Ets family regulatory transcription factor, and previous studies have shown that ERG immunohistochemistry is relatively specific for vascular tumors, among mesenchymal tumors. However, developmental biologic research has also shown ERG to play a role in chondrocyte differentiation and joint formation. We studied ERG expression in a large series of chondrogenic bone and soft tissue tumors to assess the value of ERG as a possible marker of chondroid differentiation

Design: Formalin-fixed, paraffin embedded whole sections from 103 bone and soft tissue tumors with chondroid differentiation or a morphology that may mimic cartilaginous differentiation were retrieved from our archives. Immunohistochemistry was performed using mouse anti-ERG monoclonal antibody directed against the N-terminus. Nuclear staining was scored as negative $(<5 \%), 1+(5-25 \%), 2+(26-50 \%), 3+(>51 \%)$. Any case with at least $1+$ staining was considered positive. Only cases with positive internal controls (normal endothelial cells) were scored.

Results: All cases of conventional chondrosarcoma (11/11), chondromyxoid fibroma (7/7), chondroblastic osteosarcoma (6/6), and clear cell chondrosarcoma (1/1) were positive for ERG. In cases of dedifferentiated chondrosarcoma, the well differentiated component was positive in $78 \%(7 / 9)$ of cases, while all dedifferentiated foci were negative. In cases of mesenchymal chondrosarcoma, the chondroid component was positive in $100 \%(3 / 3)$ of cases while the primitive component in all cases was negative. Variable positivity was identified in mixed tumors ( $2 / 11$ positive), extraskeletal myxoid chondrosarcomas (4/9 positive) and chondroblastomas (2/8). Only 1 of 11 cases of chordoma was positive for ERG (1+ staining). Interestingly, 15 of 18 enchondromas were negative for ERG.

Conclusions: In this study we have shown relatively constant nuclear ERG expression in selected chondrogenic tumors including conventional chondrosarcoma, chondromyxoid fibroma, chondroblastic osteosarcoma and clear cell chondrosarcoma. While ERG may be a helpful ancillary tool in certain diagnostic scenarios including the differential of conventional chondrosarcoma and chordoma, its utility may be limited as the specificity 
of this marker seems to be decreasing. The low frequency of ERG expression in enchondromas compared with conventional chondrosarcomas is interesting and may be related to the state of chondrocyte differentiation in these tumors. Further study is necessary to determine whether ERG may be useful in differentiating enchondromas from low grade conventional chondrosarcomas.

102 Kaposiform Hemangioendothelioma (KHE) Presenting as a Primary Tumor of Bone: A Clinicopathologic and Radiographic Study of 5 Cases

W Shon, DE Wenger, CY Inwards. University of Florida, Gainesville, FL; Mayo Clinic, Rochester, MN

Background: Kaposiform hemangioendothelioma (KHE) is a rare vascular tumor of intermediate (borderline) malignancy, typically involving the skin and soft tissues of the extremities, head \& neck or retroperitoneum. While KHE of the deep soft tissues can occasionally erode into underlying bone, there are few reports of KHE presenting as a primary bone tumor. We reviewed 5 cases of primary bone KHE to evaluate its clinicopathologic and radiographic features.

Design: 5 cases diagnosed as KHE were retrieved from our institutional and consultation archives. H\&E slides, radiographs (4 cases) or radiologic records (1 case), and clinical information were reviewed. For inclusion in this study, tumors had to be arising from bone and demonstrate the typical histologic features of KHE. Glut-1 immunohistochemistry was performed in 1 case.

Results: All 5 cases met inclusion criteria and included 3 females and 2 males $(10$ mos to $24 \mathrm{yrs}$, mean age $8 \mathrm{yrs}$ ). The tumors occurred in the metacarpal, humerus, ulna, distal femur and sacrum. To the best of our knowledge, none of the patients had KasabachMerritt syndrome. Radiographically, the tumors appeared benign. Histologically, all tumors showed typical features of KHE characterized by infiltrating nodules of small, compressed vessels associated with dense stroma. The neoplastic cells were relatively monomorphic and foci simulating glomeruloid structures were present. A Glut-1 stain was negative in the single tested case. Follow up was available in 2 patients. One patient received interferon therapy immediately following diagnosis and was alive with stable residual tumor at 3 years. One patient, treated with curettage and resection, developed recurrence at 6 years and was treated with pre-operative chemotherapy and surgical resection. One year later he developed multiple bone and soft tissue tumors. At latest follow-up, he was alive with residual disease.

Conclusions: KHE very rarely presents as a primary bone tumor. Similar to its extraosseous counterpart, KHE of bone most commonly occurs in children. The tumors have a benign radiographic appearance. None of our ossesous KHE tumors were associated with Kasabach-Merritt syndrome, a common association with soft tissue KHE. Improved recognition of this rare tumor involving bone will elucidate its behavior and avoid misdiagnosis with histologic mimics such as juvenile hemangioma.

103 Overexpression of ETS-Family Transcription Factors in CICDUX4 Sarcomas: A Useful Diagnostic Adjunct?

SC Smith, N Palanisamy, E-YK Choi, S Schuetze, JB McHugh, DR Lucas, D Thomas, RM Patel. University of Michigan, Ann Arbor, MI.

Background: Among small round cell sarcomas, recent reports identify a novel, highly malignant class harboring a recurrent genomic rearrangements $\mathrm{t}(4 ; 19)$, CIC-DUX4. Studies in vitro suggest that the CIC-DUX4 fusion transgene may induce expression of multiple transcription factors of the ETS family, recapitulating the oncogenesis of ETSfusions characteristic of Ewings family tumors (EFTs) and prostatic adenocarcinoma. Available antibodies for these proto-oncogenes are unsuitable for use as diagnostic adjuncts.

Design: We employed a recently validated, chromogenic RNA in situ hybridization (RISH) strategy for detection of ETV1, ETV4, and ETV5, evaluating a tissue microarray and archival whole sections of 6 confirmed CIC-DUX4 sarcomas and up to $45 \mathrm{t}(4 ; 19)$ negative lesions, predominantly EFTs $(\mathrm{N}=43)$. The stain was scored as $1+$ if present in $>25 \%$ of cells, $2+$ if present in $25-50 \%, 3+$ if diffuse $>50 \%$.

Results: The table below summarizes the results by case and stain score. Considering 1+ stain as positive, sensitivity for CIC-DUX4 sarcomas for ETV1, ETV4, and ETV5 was $100 \%$, and specificity ranged from $94-97 \%$. One EFT showed focal expression of all three ETS family members. Two poorly differentiated synovial sarcomas were uniformly negative.

Conclusions: We identified expression of ETV1, ETV4, and ETV5 in situ in CIC-DUX4 sarcomas in a robust assay using routine paraffin sections. The sensitivity and specificity observed imply potential utility as a diagnostic adjunct, especially as rare focal positivity among EFTs would be excluded definitionally by detection of EWSR1 rearrangement.

\begin{tabular}{|c|c|c|c|c|c|}
\hline Case & Age/Sex & Anatomic Site & ETV1 & ETV4 & ETV5 \\
\hline 1 & $25 / \mathrm{F}$ & Right Calf & 3 & 3 & 3 \\
\hline 2 & $32 / \mathrm{F}$ & ight Buttock & 2 & 2 & 1 \\
\hline 3 & $20 / \mathrm{F}$ & Left Shoulder & 2 & 3 & 3 \\
\hline 4 & $43 / \mathrm{M}$ & Left Knee & 3 & 3 & 3 \\
\hline 5 & $38 / \mathrm{M}$ & Lower Arm & 1 & 1 & 1 \\
\hline \multirow[t]{3}{*}{6} & $23 / \mathrm{F}$ & Left Upper Back & 1 & 3 & 3 \\
\hline & & Sensitivity $(\geq \mathbf{1}+)$ & $100 \%$ & $100 \%$ & $100 \%$ \\
\hline & & Specincity & $97 \%$ & $94 \%$ & $94 \%$ \\
\hline
\end{tabular}

104 Frequent Inactivating Mutations of the Cohesin Complex Gene STAG2 in Ewing's Sarcoma Associated with TP53 Mutations and Poor Outcome

DA Solomon, A Brohl, J-S Kim, W Chang, Y Song, JA Lopez Guerrero, I Machado, Z Wang, JJ Phillips, DH Wai, V Shahbazian, P Sorensen, AE Horvai, TJ Triche, $M$ Miettinen, A Llombart-Bosch, T Waldman, J Khan. University of California, San Francisco, CA; Mount Sinai School of Medicine, New York, NY; Georgetown University, Washington, DC; National Cancer Institute, NIH, Gaithersburg, MD; University of Valencia, Valencia, Spain; National Cancer Institute, NIH, Bethesda, MD; Children's Hospital, Los Angeles, CA; University of British Columbia, Vancouver, Canada.

Background: Ewing's sarcoma family of tumors (ESFT) are a group of malignant small round blue cell tumors most commonly arising in bone or soft tissue of children and young adults which are genetically defined by a translocation between the EWSR 1 gene on chromosome 22 with various partners, most commonly the FLI1 gene on chromosome 11. Identifying the additional driving genetic events, determining molecular subgroups associated with distinct clinical outcomes, and discovering effective targeted therapies are major focuses of current research.

Design: Whole exome sequencing of 68 ESFT primary tumors was performed, with subsequent Sanger sequencing and IHC analysis of the STAG2 gene and protein. Molecular analysis of STAG2 and TP53 in 37 ESFT cell lines was performed. Clinical data from 210 patients with ESFT was correlated with tumor STAG2 status.

Results: Inactivating mutations of the cohesin complex gene STAG2 were identified in $12 / 68$ ESFT primary tumors (18\%) and 15/37 ESFT cell lines (41\%), causing loss of STAG2 protein by IHC or Western blot using a monoclonal antibody directed at the C-terminus. Somatic mutations of STAG2 were recently reported in glioblastoma and urothelial carcinoma which were found to cause chromosomal instability and aneuploidy (Solomon et al, Science 2011 Aug 19; Solomon et al, Nature Genet 2013 Nov). We find that STAG2 mutations in ESFT are significantly correlated with the presence of chromosomal copy number aberrations and concurrent TP53 mutation. In a cohort of 210 patients with ESFT, STAG2 loss was found in 30 tumors (14\%) and correlated with reduced overall survival $(\mathrm{p}=0.03)$. Cancer cell lines harboring STAG2 mutations were found to have increased sensitivity to small molecule inhibitors of the DNA repair enzyme PARP.

Conclusions: These findings identify a molecular subgroup of ESFT defined by STAG2 mutations which harbor frequent TP53 inactivation and have poor prognosis, and suggest PARP inhibitors as a potential new effective therapy for this subgroup of ESFT.

105 A Comparative Immunohistochemical and Molecular Study of CICDUX4-Fusion Positive Round Cell Sarcomas Versus EWSR1-Rearranged Ewing Sarcomas - Further Evidence toward Distinct Pathologic Entities $K$ Specht, YS Sung, G Richter, CDM Fletcher, C Antonescu. TU München, Munich, Germany; Memorial Sloan-Kettering Cancer Center, New York, NY; Brigham and Women's Hospital, Boston, MA

Background: Round cell sarcomas harboring a t( $4 ; 19)(\mathrm{q} 35 ; \mathrm{q} 13)$ or a t $(10 ; 19)(\mathrm{q} 26 ; \mathrm{q} 13)$ with $C I C-D U X 4$ fusion are aggressive tumors arising in soft tissue of young adults. Due to morphologic overlap with Ewing sarcoma (ES) and weak CD99 expression they have been classified under ES family of tumors and managed similarly. A systematic immunohistochemical or molecular comparison between these two groups of tumors has not been conducted. On the basis of an initial observation of WT-1, FLI1 and ERG immunoreactivity in CIC-DUX4 tumors, we performed a comparative IHC and molecular analysis including these markers, to further investigate the pathogenetic relationship between ES and CIC-DUX4 tumors.

Design: 19 CIC-DUX4 sarcomas and 20 EWSR1-rearranged ES were included in the study. Tumors were evaluated by IHC for CD99, FLI1, ERG and WT-1 expression. Additionally, gene profiling by microarray techniques was carried out in CIC-DUX4 cases $(n=8)$ and gene signatures were compared with primary ES $(n=8)$ or other sarcoma subtypes ( $\mathrm{n}=30)$. QRT-PCR was used to validate microarray results.

Results: All 19/19 CIC-DUX4 cases expressed CD99 (focal and weak) and WT-1 (nuclear or nuclear and cytoplasmic positivity). They also expressed Fli1 in 8/8 cases and in $2 / 11(18 \%)$, they showed ERG positivity. In comparison, all ES tested expressed CD99 and FLI, while ERG was only seen in EWSR1-ERG-positive ES (2/2), but not in any of the EWSR1-FLI1-positive ES (0/15). WT1 was negative in all ES (0/17). Expression profiling revealed a distinct gene signature associated with $C I C-D U X 4$ sarcomas with upregulation of ETS transcription factors ETV4, ETV1 and ETV5 among the top differentially upregulated genes as compared to ES and other sarcoma subtypes. Conclusions: CIC-DUX4 sarcomas, showing weak/patchy CD99 reactivity and widespread WT1 expression, have an immunoprofile distinct from ES. WT-1 expression, although highly sensitive $(100 \%)$, is not a specific marker of CIC-DUX4 sarcoma, but can provide a useful clue in the differential diagnosis with ES, which is typically WT-1 negative. Frequent FLI1 and occasional ERG reactivity observed in CIC-DUX4 sarcoma (100\% and $18 \%$, of cases, respectively) are similarly seen in ES and represent diagnostic pitfalls. Furthermore, the gene signature of CIC-DUX4 sarcoma is distinct from classic ES, with significant upregulation of ETV4, ETV1 and ETV5 ETS transcription factors.

106 Sclerosing Epithelioid Fibrosarcoma (SEF) of the Abdominal Cavity and Retroperitoneum: An Aggressive Form of SEF Simulating Carcinomatosis

DL Stockman, JM Meis. University of Texas MD Anderson Cancer Center, Houston, TX. Background: SEF is recognized as a low to intermediate grade sarcoma that is frequently associated with dense fibrous connective tissue or periosteum. Local 
recurrences and metastases occur in approximately $50 \%$ of cases, usually over a protracted period of time. A relationship to low grade fibromyxoid sarcoma (LGMFS) has been postulated.

Design: Fifteen patients with SEF involving the abdominal cavity and retroperitoneum were identified from the files of a cancer referral center. Clinical, histologic, immunohistochemical and molecular features were analyzed.

Results: There were 8 males and 7 females, $19-79$ years of age, who presented with intra-abdominal tumors involving omentum, mesentery, retroperitoneum and/or gastrointestinal tract. Nine cases involved multiple sites, simulating carcinomatosis and 8 were associated with ascites. Tumor sizes ranged from $2-50 \mathrm{~cm}$ (median 17.5 $\mathrm{cm}$ ). All cases were highly cellular with rounded to oval small cells arranged in nests and cords by delicate to coarse strands of collagen. No areas of LGFMS were seen. EWSR1 gene rearrangement was identified in 4 cases; FUS was not detected in two cases. Follow-up was available in 12 patients ranging from 6- 73 mos (median 14 mos); 7 patients died of disease and 5 were alive with disease at median intervals of 17 and 9 mos, respectively. All patients alive with disease had lung metastases.

Conclusions: SEF occurring within the abdominal cavity simulates carcinomatosis and is therefore difficult to diagnose both clinically and pathologically. Mesothelioma and GIST must also be excluded as well as unusual presentations of Ewing sarcoma and other small cell malignancies. SEF arising in the abdominal cavity are more aggressive than those arising in the chest wall and extremities. The distinctive clinical presentation, lack of LGFMS-like areas, presence of EWSR 1 and lack of FUS gene fusion rearrangements in these cases indicate that "pure" cases of SEF are not a subtype of LGFMS, but rather a distinctive and potentially more aggressive sarcoma.

107 Gastrointestinal Neuroectodermal Tumor (GNET)/Clear Cell Sarcoma-Like Tumor of the Gastrointestinal Tract (CCSLTGT): A Clinicopathologic and Molecular Study of 41 Cases

DL Stockman, EV Zambrano, S Suster, JC Chang, AJ Lazar, JL Hornick, P Shenjere. The University of Texas MD Anderson Cancer Center, Houston, TX; Medical College of Wisconsin, Milwaukee, WI; Brigham and Women's Hospital, Boston, MA; The Christie NHS Foundation Trust, Manchester, United Kingdom.

Background: GNET/CCSLTGT is a rare gastrointestinal tumor frequently confused with its soft tissue counterpart, Clear Cell Sarcoma of Tendons and Aponeuroses(CCSTA), because of their shared molecular features despite morphologic, immunophenotypic, ultrastructural and behavioral differences. Previous studies of GNET/CCSLTGT have had too few cases or too little follow-up to provide a comprehensive pathologic and clinical picture of the tumor.

Design: 41 tumors with features of GNET/CCSLTGT as had been previously described were identified and were assessed by either fluorescence in-situ hybridization(FISH) for EWSR1 rearrangement or RT-PCR for EWSR1-ATF1 and/or EWSR1-CREB1 fusion transcripts. Cases were included if there was adequate histologic material and clinical information.

Results: GNET ( $\mathrm{N}=41$ ) occurred in 22F/19M (median $46 \mathrm{yrs)}$ in various gastrointestinal (GI) locations (median size $4 \mathrm{~cm}$, range $2-15 \mathrm{~cm}$ ). Tumors involved the stomach(N=6), small intestine $(\mathrm{N}=24)$, colon $(\mathrm{N}=4)$, rectum $(\mathrm{N}=1)$, omentum and mesentery $(\mathrm{N}=5)$. The typical histologic findings were sheets of highly cellular, polygonal cells with slight nuclear pleomorphism and variable mitotic activity(median 4, range 1-30). Scattered, osteoclast-type giant cells and clear cell morphology was present in less than half the cases. Abortive rosette-like structures were seen in 18 cases. EWSR1 gene rearrangement was present in 32/35 (91\%) cases, EWSR1-ATF1 in 10/25 cases and EWSR1-CREB1 in $8 / 25$ cases. Ten patients died of their tumor after 3 to 115 months (median 31 months). Eighteen patients were alive at latest follow-up of 2 to 93 months (median 23 months), 6 with tumor and 12 without. Eight patients had recurrence, and 15 had metastases (mostly in liver) after periods of up to 107 months. Histologic differences, mitotic activity, and tumor size were not related to tumor behavior or patient survival.

Conclusions: GNET has a range of morphologic, immunophenotypic, ultrastructural and behavioral differences that separates it from CCSTA.

\section{Granular Cell Tumors (GCT) of Soft Tissue: A Clinicopathologic}

\section{Study of 35 Cases}

J Stone, JA Plaza, S Suster, E Zambrano. Medical College of Wisconsin, Milwaukee, WI. Background: GCTs are benign neoplasms of neurogenic origin commonly arising from superficial anatomical locations.

Design: GCTs involving deep soft tissues (i.e. +/- deep dermis and below) with no superficial dermal/mucosal involvement were selected. Clinical and follow-up data was obtained. H\&E sections and immunostains including S-100, EMA, CD34, SMA and MOC 31 were analyzed in all cases.

Results: 35 cases ( 21 female, 14 male; ages: 5-69 years) were identified: arm (7), chest (7), hand (5), axilla (3), leg (3), foot (2), abdomen (2), scalp (2), cheek (1), vulva (1), back (1), finger (1) (size: $1-3.5 \mathrm{~cm}$; median: $2.25 \mathrm{~cm}$ ). Histologically, 22 tumors had a nodular well-circumscribed appearance, 9 tumors had infiltrative borders and 4 tumors were multinodular. Tumors were composed of sheets and nests of oval to polygonal cells with abundant, finely granular, eosinophilic cytoplasm with well-defined cytoplasmic borders, small uniform round to oval nuclei with evenly dispersed chromatin and indistinct nucleoli; 21 cases showed a pure epithelioid appearance, 12 cases showed a mixed epithelioid/spindled appearance and 2 cases had a pure spindle cell appearance. Nine cases showed occasional nuclear pleomorphism; 9 showed perineural invasion and 1 had lymphovascular invasion. Only 2 cases showed rare mitotic figures ( 1 per $10 \mathrm{HPF}$ ) with no atypical mitoses. No necrosis or hemorrhage was identified in any case. All tumors were strongly positive for $\mathrm{S} 100$ protein and negative for all other markers. Follow up from 2 months-4 years revealed only one patient with multiple, multifocal recurrences (35 year-old male; $3 \times 2 \mathrm{~cm}$, arm). No atypical histological features, or perineurial or vascular invasion were seen on initial presentation or upon recurrence.
Conclusions: None of the cases behaved as aggressive malignant neoplasms in spite of their deeper anatomical locations. The presence of occasional atypical features, such as cellular pleomorphism (25.7\%), perineurial invasion $(25 \%)$, mitotic activity $(5.7 \%)$ and lymphovascular invasion $(2.8 \%)$, did not influence prognosis in these deep-seated tumors.

\section{Osteomyelitis: Development of a Scoring System Based on} Histologic Criteria to Improve Diagnosis in the Foot and Ankle

AB Sybenga, D Jupiter, A Rao. Baylor Scott \& White Memorial Hospital/Texas A\&M Health Science Center, Temple, TX.

Background: Histopathologic exam of bone specimens is the gold standard of diagnosis for osteomyelitis (OM); however, strict criteria are not widely recognized. Accurate diagnosis of acute OM (AOM) and chronic OM (COM) is important for optimal treatment of patients. We developed a scoring system based on histologic criteria for $\mathrm{AOM}$ and COM, and correlated it with clinical follow up of OM in foot and ankle for the 2010 to 2013 period.

Design: 750 cases over 3 years were identified. Clinical, radiologic, laboratory and histopathologic findings were collected. From these findings and literature, we developed and weighted 13 histologic criteria to test the feasibility of improving diagnosis of OM. Acute inflammation, abscess, bacteria, involucrum, and sequestrum are the most supported findings in $\mathrm{OM}$ and were assigned a score of 3 . Fibrinoid necrosis and marrow fibroplasia, were assigned a score of 2 . Nonspecific findings, including erosion, remodeling and chronic inflammation, were assigned a score of 1 . A slide review was then performed with strict adherence to the criteria. Also included in the score were radiologic results ( 2 for AOM, 1 for suspicious for OM (SOM)), and bone culture (1 for positive)

Results: A review of previously issued reports indicated $61 \%$ utilized descriptive criteria, none of which included complete criteria. $18 \%$ of amputations had no margins submitted. Bone cultures were performed in only $14 \%$ of cases. The average score for $\mathrm{AOM} / \mathrm{ACOM}$ was 14.89 with $87 \%$ of cases scoring above 11 . The average score for COM was 8.95 with $81 \%$ scoring between 6 and 11 . The average score for no acute OM (NAOM) was 1.94 with $100 \%$ scoring 4 or less. No diagnosis of AOM/ACOM scored less than 9 , and no diagnosis of COM scored less than 6. COM was diagnosed in $25 \%$ of cases, compared to $6 \%$ without the scoring system. Clinical follow up results indicate $50 \%$ of reamputations and non-healing post-op wounds might have been avoided using our criteria. The criteria were particularly helpful in evaluating margins and fragmented biopsies.

Conclusions: Review of reports indicates that current histopathologic criteria for diagnosis of OM is ill defined and inconsistently utilized. Our scoring system shows scores greater than 6 are likely OM, scores greater than 11 are likely AOM, and scores less than 4 are NAOM. Bone cultures are infrequently utilized, but seem to correlate more highly with a diagnosis of AOM.

110 Quantitative miRNA Expression Profiling as an ObjectiveTool to Differentiate Malignant Peripheral Nerve Sheath Tumors and Desmoplastic Melanomas

SK Tian, PP Koty, OP Sangueza, MG Trizna, SA Qasem. Wake School of Medicine, Winston-Salem, NC; Smithsonian Institute, Washington, DC.

Background: Differentiating malignant peripheral nerve sheath tumor (MPNST) from desmoplastic melanoma (DM) can be a diagnostic challenge due to overlapping morphology and immunophenotype. We have recently demonstrated through microarray analysis that miR-138 is differentially expressed and can be used to differentiate these two entities. The aim of this current study is to expand our sample size and confirm our findings using quantitative polymerase chain reaction (qPCR). Initial global miRNA expression profiling was performed on archival formalin-fixed paraffin-embedded tissue. Then a limited panel of candidate miRNAs was converted to a qPCR format for practicality and economy.

Design: A scrreening group of 7 MPNST and 8 DM cases with sufficient tissue was selected. Each case was independently confirmed by a soft tissue pathologist and a dermatopathologist. Universal miRNA expression was analyzed with the GeneChip miRNA 3.0 Array (Affymetrix Inc). Data was imported into R software and normalized using the robust multichip algorithm. Differential expression was assessed using the Wilcoxon signed-rank test. Significant human probe sets by microarray were identified based on a significant $\mathrm{p}$-value $(<0.05)$ and a difference of at least \pm 1.5 fold-change. Candidate miRNAs demonstrating the most significant difference were selected (miR138, mir-141, and miR-200a). A candidate miRNA demonstrating the least variance was selected as a calibrator (miR-892b). Total RNA was used for selective miRNA qPCR (Qiagen Inc). Data was analyzed for relative expression fold change using the $2^{-\Delta \Lambda} C_{\mathrm{T}}$ method.

Results: MiR-138 demonstrated the most significant relative fold change both on microarray $(\mathrm{p}=0.02)$ and $\mathrm{qPCR}(\mathrm{p}=0.006)$. MiR-141 and miR-200a showed a significant relative difference on the microarray; however, the trend could not be confirmed with qPCR (see table).

Relative Expression Fold Difference in MPNST and DM
\begin{tabular}{|l|l|l||}
\hline miRNA & $\begin{array}{l}\text { Microarray; MPNST relative } \\
\text { to DM (p-value) }\end{array}$ & $\begin{array}{l}\text { QPCR; MPNST relative to } \\
\text { DM (p-value) }\end{array}$ \\
\hline \hline hsa-miR-138 & $1.64(0.029)$ & 6.00 (0.006) \\
\hline ha-miR-141 & $-2.31(0.040)$ & $-2.85(0.309)$ \\
\hline hsa-miR-200a & $-2.02(0.029)$ & $-2.59(0.425)$ \\
\hline
\end{tabular}

\begin{tabular}{|l|l|}
\hline hsa-miR-200a & $-2.02(0.029)$ \\
Conclusions: The data supports our previous work suggesting low expression of
\end{tabular} miR-138 in DM relative to MPNST. A targeted miRNA qPCR multiplex panel can be developed and used, in a clinically meaningful way, as a tool for accurate classification of diagnostically challenging cases. This may also help shed light on the pathobiology of these tumors and any possible therapeutic targets. 


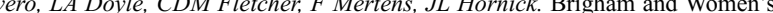
Hospital and Harvard Medical School, Boston, MA; University and Regional Laboratories, Skane University Hospital, Lund University, Lund, Sweden.

Background: Solitary fibrous tumor (SFT) is a fibroblastic neoplasm characterized by a haphazard growth pattern, bland, spindled cytomorphology, variable amounts of collagenous stroma, and dilated, branching vessels. Individual tumor histology, however, can vary greatly and overlap with other soft tissue tumors, making diagnosis challenging at times. Several existing markers, among which CD34 has been studied most extensively, are used for diagnosis of SFT, but none are specific, and all have been shown to stain histologic mimics of SFT. Recent gene expression profiling studies have revealed marked overexpression of the GRIA2 gene (GluR2), which encodes an AMPA-selective ionotropic glutamate receptor subunit, in SFT. To evaluate the potential diagnostic utility of GRIA2, we examined protein expression in SFT and other soft tissue tumors, including histologic mimics.

Design: Paraffin-embedded whole tissue sections of 375 soft tissue tumors were examined, including 105 solitary fibrous tumors, 20 cases of dermatofibrosarcoma protuberans (DFSP), 9 myoepitheliomas, 20 sarcomatoid mesotheliomas, 20 malignant peripheral nerve sheath tumors, 25 dedifferentiated liposarcomas, 20 monophasic synovial sarcomas, 22 low grade fibromyxoid sarcomas, 12 spindle cell/sclerosing rhabdomyosarcomas, 10 nodular Kaposi sarcomas, 10 cases of desmoid fibromatosis, 20 gastrointestinal stromal tumors, 10 cellular schwannomas, 11 deep fibrous histiocytomas, 21 spindle cell lipomas, 10 neurofibromas, and 20 soft tissue perineuriomas. Immunohistochemistry was performed using a rabbit anti-GRIA2 monoclonal antibody (Abcam; EP929Y; 1:100 dilution) following pressure cooker antigen retrieval. Results: In total, 84 of $105(80 \%)$ SFT, including 18 of $21(86 \%)$ malignant SFT and 4 of $4(100 \%)$ dedifferentiated SFT, demonstrated positivity for GRIA2, which was usually moderate or strong in intensity and present in at least $25 \%$ of tumor cells. Expression of GRIA2 was also seen in 15 of $20(75 \%)$ DFSP, 4 of $9(44 \%)$ myoepitheliomas, one monophasic synovial sarcoma ( $<1 \%$ of cells), and one cellular schwannoma. No other soft tissue tumors were positive for GRIA2.

Conclusions: GRIA2 is a useful diagnostic marker to distinguish SFT from most histologic mimics. Among other CD34-positive tumors, GRIA2 is also positive in most DFSP; however, clinical and histologic features aid in their distinction from SFT. GRIA2 shows a very limited distribution in other soft tissue tumors.

\section{HMGA2 Expression Is Useful in the Diagnosis of Spermatic Cord} Fatty Lesions

DP Wang, J Schowinsky, JA Wisell. University of Colorado School of Medicine, Aurora, CO.

Background: Well differentiated liposarcomas (WDL) of the spermatic cord are rare tumors often found incidentally. These may be difficult to recognize and may be misclassified as a 'cord lipoma.' The advent of immunohistochemical (IHC) antibodies against novel antigens has provided a diagnostic tool. IHC antibodies against HMGA2 label a large proportion (up to $86 \%$ ) of lipomas and WDLs. We postulate that the large majority of 'cord' lipomas are not truly lipomas at all, but rather extensions of nonneoplastic preperitoneal adipose tissue into the inguinal canal; as such, they would not be expected to express HMGA2, furthering the diagnostic utility of this antibody. Design: From our files we collected 14 cases diagnosed as 'cord lipoma' and 4 cases diagnosed as lipomas from other locations. We reviewed the morphologic features for all 18 cases. Specifically, we assessed cellular size variability, nuclear pleomorphism and nuclear hyperchromasia of the lesional adipocytes and vascular prominence. HMGA2 IHC studies were performed on all 18 cases.

Results: All 14 'cord lipoma' cases were entirely negative for HMGA2 and contained large muscular vessels within the lesions. All 4 lipomas from other locations had diffuse and strong HMGA2 labeling. Only one of the lipoma cases contained any large vessels within the lesion. All 18 cases lacked nuclear hyperchromasia and pleomorphism. 3 out of 14 'cord lipomas' and 2 out of 4 lipomas displayed cellular size variation.

Conclusions: Our findings support previous anatomic studies that dismiss the longstanding practice of designating fatty deposits within the spermatic cord as 'lipomas.' We found that the presence of muscular vessels within the lesion to be a distinguishing feature of spermatic cord fatty deposits, a feature not typically seen in lipomas. None of the cord lipoma cases exhibited HMGA2 labeling, indicating a different pathogenesis than found in neoplastic adipocytic tumors. In addition to informing the nosology, these findings support the role of HMGA2 as a diagnostic tool when evaluating spermatic cord fatty lesions.

113 Genetics of Melanotic Schwannomas - A Pilot Study by SNP-Array Analysis

L Wang, J Zhou, $N$ Agaram, T Patel, M Rosenblum, M Hameed. Memorial SloanKettering Cancer Center, New York, NY.

Background: Melanotic schwannomas are rare tumors of adulthood with a peak incidence in the $4^{\text {th }}$ decade of life. Derived from neuroectoderm, several theories have been proposed regarding their etiology including melanomatous transformation of Schwann cells, phagocytosis of melanin by Schwann cells and simultaneous presence of two neoplastic populations of proliferating Schwann cells and melanocytes. The tumors occur in the spinal canal, paraspinal regions and rarely in organ systems such as the GI tract. While majority of the melanotic schwannomas behave of an indolent manner, up to $24 \%$ can metastasize and there are no morphological criteria which help distinguish the indolent from aggressive tumors. The genetics of this lesion are largely unknown. The objective of this project is to retrospectively study available melanotic schwannomas at MSKCC and analyze their genetic characteristics by array-based DNA copy number analysis.

Design: Nine cases were identified and in 5 cases FFPE tissue was available for genomic analysis. There were 6 males and 3 females. Median age was 53 years. Tumors were located in chest wall (2), vertebra (2), paraspinal region (2), sacrum (1), ischium (1) and metacarpal bone (1). Tumor size ranged from $2.5 \mathrm{~cm}$ to more than $7 \mathrm{~cm}$. Genomic DNAs was extracted from FFPE tumor material and Affymetrix OncoScan SNP-array was used for copy number and allelic imbalance analysis.

Results: SNP-array analysis on 5 cases revealed non-random loss of multiple chromosomes, and the most common recurrent abnormalities were loss of chromosomes 1,2 and 17; other recurrent abnormalities included loss of chromosomes 3 and 21. Allelic imbalance on chromosome 17q was observed in all 5 cases, three of which presented as deletion and two cases showed copy-neutral loss of heterozygosity (CN-LOH) of 17q. It is worth noting that one case showed multiple chromosomal gains rather than loss, however, multiple $\mathrm{CN}-\mathrm{LOH}$ was also identified in other chromosomes including 1, 2, 3,17 and 21 . Therefore, the combined copy number and allelic imbalance in this case indicate that the genomic abnormalities may result from doubling of a hypodiploid clone. Conclusions: Hypodiploidy is a common genetic feature of Melanotic Schwannoma, and typically involves monosomies of chromosomes 1, 2, and 17. Allelic imbalance on $17 \mathrm{q}$ is the single most common aberration observed in this study, which presents as deletion or copy-neutral $\mathrm{LOH}$ of $17 \mathrm{q}$

\section{Multiple Sporadic Desmoid Fibromatosis: Clinical and Mutational} Analysis

W-L Wang, C Colombo, K Torres, AJ Lazar. The University of Texas MD Anderson Cancer Center, Houston, TX; Fondazione IRCCS Istituto Tumori di Milano, Milano, Italy.

Background: Desmoid fibromatosis is a benign myofibroblastic proliferation often characterized by mutations in CTNNB1 in sporadic cases. Those associated with germline $A P C$ mutations (with familial adenomatous polyposis, FAP) are often multifocal. However, non-FAP related tumors can also rarely be multi-focal either regionally or at distant body sites. We examined a series of such cases and their clinical course. Design: Twenty patients with sporadic multifocal desmoid fibromatosis (without FAP) were identified from prospective databases at two institutions. Demographics, number of tumors, sites involved, CTNNBI mutational analysis, and clinical follow-up were tabulated.

Results: The median age was 39 (range:15-84) years with slight female predominance (M:F 8:12). Sites of involvement included: trunk (10), extremities (6), chest (4), intra-abdominal (3), head and neck(2) and abdominal wall (1), with 7 patients having multiple tumors at different sites. Number of tumors $(\mathrm{n}=12)$ ranged from 2 to 3 . Median size $(\mathrm{n}=14)$ was $5 \mathrm{~cm}$ (range:0.4-24). 12/19 cases developed local recurrence. Follow -up was available in 19 patients: 14 alive with disease, 4 alive and well, and one dead likely from disease. CTNNB1 mutational analysis was available on multiple nodules in four cases: in 2 cases (one extremity and one intra-abdominal) both tumor foci revealed T41A; while two multi-focal intra-abdominal cases show 2 different results for CTNNB1 genotyping (S45P and WT or T41)

Conclusions: Patients can rarely have sporadic multifocal desmoid fibromatosis and interestingly, these tumors can harbor different mutations, arguing that they are separate clonal events. This finding raises the possibility that some patients may be prone to develop CTNNB1 mutations.

\section{Immunohistochemical Study of BRAF V600E Mutant Protein} Expression in High-Grade Sarcomas

$K$ Whiting, A Valente, J Tull, C Maciak, S Zhang. SUNY Upstate Medical University, Syracuse, NY.

Background: V-raf murine sarcoma viral oncogene homolog B1 (BRAF), a serinethreonine protein kinase, is a member of the RAS-REF-MEK-ERK signaling pathway. The BRAF $\mathrm{V} 600 \mathrm{E}$ has been considered as a cancer-driving mutation in a variety of neoplasms including melanomas, thyroid carcinomas, gastrointestinal stromal tumors, colorectal carcinomas, ovarian carcinomas and lung cancers. Zalboraf, a FDA approved $B R A F \mathrm{~V} 600 \mathrm{E}$ kinase inhibitor, has been applied in treatment of melanoma harboring $B R A F$ V600E with considerable response. Although a few studies have been done in sarcomas including Ewing sarcomas and rhabdomyosarcomas, the status of $B R A F$ V600E in high-grade sarcomas remains unclear. In addition, current immunohistochemistry (IHC) with VE1 antibody for $B R A F$ V600E protein is reported highly specific and sensitive to predict $B R A F$ V600E DNA mutation, but its utility on high-grade sarcomas is unknown.

Design: Forty-eight cases of undifferentiated high grade sarcomas were constructed in duplicate or triplicate cores using tissue microarray technology. IHC with VE1 antibody against $B R A F$ V600E protein (Spring Bioscience, 1:50) was performed as per manufacturer's instructions. The microarrays were independently reviewed by two board-certified pathologists and one pathology resident. IHC staining intensity in cytoplasm was graded from 0 to $3+.0$ and $1+$ were considered as negative while $2+$ or greater as positive. Selected cases were tested for $B R A F \mathrm{~V} 600 \mathrm{E}$ gene mutation by real-time PCR for comparison.

Results: Forty-one out of 48 specimens remained intact in both cores after IHC processing. Six of the 41 cases $(15 \%)$ were scored as positive $(2+$ or $3+)$ by consensus. These 6 cases were subsequently tested for the BRAF V600E mutation by PCR, and they were all negative. Additionally, weak nuclear staining, which was considered nonspecific, was seen in $14 \%(12 / 88)$ of cores examined. 
Conclusions: Although IHC with VE1 antibody was previously reported as highly sensitive and specific for $B R A F \mathrm{~V} 600 \mathrm{E}$ in other neoplasms, it shows significant non-specific expression in high grade sarcomas, and caution should be exercised in interpreting the results. Nonetheless, $B R A F \mathrm{~V} 600 \mathrm{E}$ mutation seems rare in high-grade sarcomas.

\section{PTENExpression in Rhabdomyosarcoma:An Immunohistochemistry Study}

EM Williams, MN Svalina, TK Morgan, C Keller, A Mansoor. Oregon Health \& Science University, Portland, OR.

Background: PTEN (phosphatase and tensin homolog) acts as a tumor suppressor protein through inhibition of the PI3K-AKT-mTOR pathway. It regulates multiple cellular processes including cell survival and proliferation. Loss of PTEN leads to absence of this inhibitory signal and a variety of malignancies. Small molecule inhibitors that target members of the PI3K-AKT-mTOR pathway are available and may be of therapeutic benefit in tumors, which have lost PTEN inhibitory function. To date, we are aware of only rare studies evaluating loss of PTEN in rhabdomyosarcoma (RMS), including only one that directly evaluated tissues from a limited number $(\mathrm{n}=12)$ of human subjects. Our objective was to test for the loss of PTEN in human RMS cases by using immunohistochemistry.

Design: We retrospectively identified 222 archival cases of RMS specimens submitted for surgical pathology diagnosis. These cases were previously diagnosed as ARMS ( $\mathrm{n}$ =72), ERMS $(\mathrm{n}=123)$, PRMS $(\mathrm{n}=18)$, and URMS $(\mathrm{n}=9)$ (alveolar, embryonal, pleomorphic, and undifferentiated subtypes, respectively). Representative sections were immunostained for PTEN and signal graded as absent or intact.

Results: PTEN immunostaining was lost in the majority of RMS cases (186/222, 84\%). When stratified by tumor type, there was no statistically significant difference in PTEN expression between subtype groups [table]. However, comparison of PRMS to all other RMS groups combined revealed a tendency toward retention of PTEN in PRMS $\left(\mathrm{X}^{2}\right.$ p-value 0.04; Fisher's exact p-value 0.08)

PTEN Expression by RMS Subtype

\begin{tabular}{|c|c|c|c|c|c|}
\hline Tumor & ARMS & ERMS & PRMS & URMS & Total \\
\hline PTEN intact & $14(19.4 \%)$ & $15(12.2 \%)$ & $6(33.3 \%)^{*}$ & $1(11.1 \%)$ & $36(16.2 \%)$ \\
\hline PTEN loss & $58(80.6 \%)$ & $108(87.8 \%)$ & $12(66.7 \%)$ & $8(88.9 \%)$ & $186(83.8 \%)$ \\
\hline Total & $72(100 \%)$ & $123(100 \%)$ & $18(100 \%)$ & $9(100 \%)$ & $222(100 \%)$ \\
\hline
\end{tabular}

Conclusions: The majority of RMS cases demonstrated loss of PTEN staining by IHC, which identifies a significant subset of RMS patients that may therapeutically benefit from PIK3-Akt-mTOR inhibitors. We observed a potentially significant trend toward increased retention of PTEN in PRMS, suggesting that PIK3-Akt-mTOR pathway may be less contributory in this subtype. A limitation of the current study is the lack of molecular genetic data for FOXO1 in early archival cases. Fusion proteins resulting from FOXO1 rearrangements have been shown to downregulate PTEN in RMS cell lines, thus translocation positive tumors may be more likely to show loss of PTEN.

\section{Primary Sclerosing Epithelioid Fibrosarcoma of Bone: Analysis of a Series}

JB Wojcik, JL Hornick, AM Bellizzi, P Dal Cin, CDM Fletcher, GP Nielsen. Massachusetts General Hospital, Boston, MA; Brigham and Women's Hospital, Boston, MA; University of Iowa, Iowa City, IA

Background: Sclerosing epithelioid fibrosarcoma (SEF) is a rare, aggressive malignant neoplasm characterized by small nests and linear arrays of epithelioid cells embedded in a dense collagenous stroma. Very few primary SEF of bone have been reported. Recognition is critical, as the dense collagenous matrix can be interpreted as osteoid, leading to misdiagnosis as osteosarcoma. MUC4 has recently been identified as a novel diagnostic marker for SEF; MUC4 is consistently negative in osteosarcoma. The purpose of this study was to characterize the clinicopathologic and immunohistochemical features of SEF primary in bone.

Design: Eight primary SEF of bone were identified from case files of three institutions. The clinical and pathological features of the tumors including their histologic and immunohistochemical characteristics were analyzed.

Results: The patients included 5 males and 3 females, aged 25-73 (median 52) years. The original diagnoses were: SEF (4), small-cell osteosarcoma (2), sclerosing epithelioid leiomyosarcoma (1) and low-grade chondroid neoplasm(1). The tumor locations were: femur (3), ulna (2) (1 parosteal), humerus (1), C6 (1) and metacarpal (1). Imaging studies showed a destructive appearance, with extension into adjacent soft tissues. Microscopically, the tumors contained loosely cohesive cells in small clusters and cords, infiltrating through a collagenous stroma. No mineralization was seen. The cells were predominantly epithelioid, though foci of spindled morphology with vaguely fascicular growth were present in several cases, and one case contained a component of low-grade fibromyxoid sarcoma. Immunohistochemically, 6/8 strongly expressed MUC4, 2/8 were positive for SATB2, 3/6 showed at least focal reactivity for EMA, 2/7 at least focal keratin staining and 1/7 focal smooth-muscle actin expression. Both cases that showed SATB2 expression were also positive for MUC4, allowing for distinction between SEF and osteosarcoma. One case harbored EWSRI rearrangement. Treatment was generally with wide local excision. Three patients developed metastases, ranging from 1 month to 7 years after primary excision.

Conclusions: SEF is a rare malignant neoplasm that may present as a primary bone tumor. Histologic features and expression of SATB2 may lead to the misdiagnosis of an osteosarcoma. Immunohistochemical staining for MUC4 is useful in confirming the diagnosis as SEF of bone, similar to its soft tissue counterpart.
118 Denosumab-Treated Giant Cell Tumor of Bone May Mimic Primary Benign and Malignant Tumors of Bone

JB Wojcik, IA Chebib, AE Rosenberg, GP Nielsen, V Desphande. Massachusetts General Hospital, Boston, MA; University of Miami Miller School of Medicine/Jackson Memorial Hospital, Miami, FL.

Background: Giant cell tumor (GCT) of bone is a locally aggressive benign neoplasm characterized by a preponderance of osteoclastic giant cells admixed with mononuclear cells. Giant cell proliferation is induced by the neoplastic mononuclear cells that express high levels of RANKL. Denosumab, a RANKL inhibitor with proven efficacy in the treatment of GCT, can lead to a marked histological alteration of the tumor leading to diagnostic difficulties. Herein we provide the first detailed histomorphologic assessment of denosumab-treated GCT.

Design: Four cases of GCT of bone in patients who had received denosumab therapy were identified from the case files at our institution. Both pre- and post-therapy materials were reviewed.

Results: The patients included two males and two females, aged 16-32 years. All presented with lytic bone lesions with extension into adjacent soft tissues. One tumor was sacral, one tibial, one in the pelvis and one in the first metacarpal bone. The time from initiation of therapy to resection/repeat biopsy/curettage ranged from 7 months to 39 months. In all cases the initial biopsy had classical GCT morphology. The posttherapy histology was highly variable. Osteoclast-type giant cells varied from sparse ( 2 cases) to absent ( 2 cases). In two cases, the histologic appearance was dominated by osteoid which was deposited both in delicate interlacing networks and in large seams. The osteoid was rimmed by a spindle cell population that showed mild to moderate atypia. Osteoblasts were conspicuously absent. The appearance was reminiscent of a low-grade osteosarcoma. One of the other lesions was characterized by a storiform fibrohistiocytic proliferation with moderate cytologic atypia and reactive bone formation. The overall appearance was suggestive of a non-ossifying fibroma. One tumor was composed of oval mononuclear cells and foam cells. The post therapy tumors lacked mitotic activity and did not infiltrate preexisting bone. In two cases the adjacent bone showed evidence of remodeling adjacent to cartilage formation with an appearance reminiscent of osteopetrosis.

Conclusions: The histological appearance of GCT of bone following denosumab therapy is highly variable, and may mimic a variety of benign and malignant tumors of bone. Careful attention to the history of denosumab therapy is crucial to avoid misdiagnosis. Features useful in the distinction between treated GCT and osteosarcoma are the absence of infiltration of pre-existing bone and mitotic activity.

119 Primary Bone Lymphoma: Prognostic Significance of Soft Tissue Extension, International Prognostic Index and Multifocality

$H$ Wu, L Zhang, H Shao, M Bui. Peking Union Medical College Hospital, Beijng, China; Moffitt Cancer Center, Tampa, FL.

Background: Primary bone lymphoma (PBL) is a rare disease. There has not been any study of prognostic factors using the new definition of primary non-Hodgkin lymphoma of bone described in the 2013 WHO Classification of Tumors of Soft Tissue and Bone. Design: We examined PBL cases at Moffitt Cancer Center from 1998 to 2013 using the 2013 WHO criteria. Patient characteristics, survival, and prognostic factors were analyzed and compared with published data in the literature.

Results: Seventy PBLs diagnosed and treated at Moffitt Cancer Center from 1998 to 2013 were found, and $53(75.7 \%)$ were histologically confirmed as primary bone diffuse large B-cell lymphoma (PB-DLBCL). Adjacent soft tissue extension and multifocal bone lesions were both common findings in PBL. The data of PB-DLBCL subgroup were further analyzed for survival. The patients with PB-DLBCL had 3- and 5-year progression free survival (PFS) of $61.2 \%$ and $46.9 \%$ respectively, while 5- and 10 -year overall survival (OS) were $81.1 \%$ and $74.7 \%$. In univariate analysis, soft tissue extension, multifocal lesions, stage IV, elevated LDH, high International Prognostic Index (IPI) score, and single-modality therapy were significant poor prognostic factors for both PFS and OS. Age $\geq 60$ and high Performance Score ( $P S \geq 2$ ) were also significant adverse prognostic factors for OS. Multivariate analysis revealed that soft tissue extension and IPI score were the most important unfavorable prognostic factors for both PFS and OS. Multifocality appeared to be highly associated with a worse PFS and OS, although it was not identified in multivariate analysis due to its incorporation into IPI.

Conclusions: This is the first study using the new 2013 WHO classification to identify prognostic indicators in PBL.PB-DLBCL patients with soft tissue extension or high IPI score were found to have a significantly poor survival. The prognostic significance of soft tissue extension needs further confirmation in larger well-characterized clinical cohorts. Moreover, further investigation is warranted to address whether PBL with multifocal bone lesions could be considered as a systemic disease rather than a conventional PBL.

120 Giant Cell Tumor of Bone with Chondroid Differentiation. A Series of 7 Cases

F Yamani, AE Rosenberg. University of Miami Miller School of Medicine/Jackson Memorial Hospital, Miami, FL.

Background: Giant cell tumor (GCT) of bone is a benign locally aggressive tumor that is composed of neoplastic mononuclear cells and benign multinucleated osteoclast type giant cells. The mononuclear cells usually grow in a syncytial pattern, the giant cells are diffusely distributed, and contain numerous usually centrally located nuclei. GCT may contain intratumoral tumor however areas of chondroid differentiation are extremely rare. In this study we describe the clinicopathologic features of GCT with chondroid areas. 
Design: 7 cases of GCT of bone that contain chondroid areas; 6 cases were derived from the consult series of one of the authors and 1 case from University of Miami Hospital. 3 cases had immunohistochemistry (IHC) for S100, 2 had p63, and 1 had EMA available for review. Radiographic information was available for 4 cases, tumor size for 2, and post resection followup for 3 .

Results: The 7 cases represent a mean age of 24 years (range 8-42), 4 males and 3 females. 4 GCT were located in the distal femur, and the remaining 3 in metatarsal, patella, and scapula. Radiographically, a radiolucent, well circumscribed mass associated with thinning of cortex was present. Tumor size ranged between 4.7 and $6.1 \mathrm{~cm}$. Histologically the tumors contained regions diagnostic of GCT as well as scattered foci of chondroid matrix. The matrix consists of cartilage which is hyaline and fibrocartilaginous in appearance and that in areas appears to be associated with bone. The matrix is surrounded by regions of tumor that have the appearance of GCT and areas where the mononuclear cells have a spindle cell morphology. Minimal necrosis and mitotic activity was observed and no cytologic atypia was observed. By IHC, 2 cases were positive for $\mathrm{S} 100$ in chondroid areas when initially diagnosed but were negative when recently repeated. 1 case was negative for S100 in the chondroid areas. In 1 case p63 was positive in the neoplastic mononuclear cells and the cells inhabiting the chondroid areas. EMA was positive in chondroid areas in 1 case. Followup at this time is limited; 1 patient is free of disease 5 years after curettage and 2 have been disease free for 4 months.

Conclusions: GCT of bone with chondroid differentiation are well circumscribed, radiolucent tumors that arise in the epiphyseal and metaphyseal regions and are extremely uncommon. The differential diagnosis of this tumor includes chondroblastoma, osteoblastoma, giant-cell rich osteosarcoma, and other cartilage containing tumors. Further investigation is required to determine the exact nature of the chondroid matrix.

121 STAT6 Immunohistochemistry Is Helpful in the Diagnosis of Solitary Fibrous Tumors

A Yoshida, K Tsuta, M Yoshida, R Kushima. National Cancer Center Hospital, Tokyo, Japan.

Background: Solitary fibrous tumor (SFT) is an uncommon fibroblastic neoplasm. Although histological characteristics and frequent CD34 expression allow for an accurate diagnosis in the majority of SFT cases, a wide histological spectrum and an occasional unexpected immunophenotype may pose diagnostic challenges. Molecular analyses have discovered that almost all SFTs harbor a NAB2-STAT6 fusion gene, which is considered specific to this tumor type. Recent studies have suggested that STAT6 immunohistochemistry is a reliable surrogate for detection of the fusion gene. Our aim was to validate these findings by examining a large number of SFT cases and a broad array of 30 different types of non-SFT tumors.

Design: A total of 49 SFTs with a range of histological characteristics (including 33 conventional cases and 16 cases with unconventional histology) and 159 benign or malignant tumors that can mimic SFTs were retrieved. A representative block from each case was stained by using STAT6 antibody that recognizes the carboxy-terminal portion of this protein (polyclonal, sc-621, dilution 1:1000; Santa Cruz Biotechnology). The intensity of staining was semi-quantitatively graded as negative, weak, moderate, or strong. The extent of staining was classified as negative $(0$ to $<5 \%)$, focal $(5-50 \%)$, and diffuse ( $>50$ to $100 \%$ ).

Results: All 49 SFTs (100\%) showed STAT6 expression that was restricted in the nucleus, irrespective of the tumor sites and histological patterns. The staining was diffuse in all but 1 case. The intensity was strong in 45 cases, moderate in 3 cases, and weak in 1 case. The staining was uniform in most cases, but was heterogeneous in about $20 \%$ of the cases where zonal staining attenuation was observed likely reflecting variability in fixation or tissue ischemia. In contrast, only 4 non-SFT tumors $(2.5 \%)$ exhibited weak nuclear STAT6 expression, while the remaining 155 cases showed no staining (81 cases) or often weak reactivity in both the cytoplasm and the nucleus (74 cases). Conclusions: Nuclear STAT6 immunoreactivity, typically in a diffuse strong manner, is highly sensitive and specific for SFTs and is diagnostically helpful. This pattern of expression likely reflects the NAB2-STAT6 fusion protein, and should be distinguished from the usually weak cytoplasmic/nuclear expression associated with full-length STAT6 that can be seen in some non-SFT tumors. STAT6 reactivity may depend on preanalytical conditions, and the quality of specimens and preparations needs to be carefully monitored if this technique is to be applied to routine diagnosis of SFTs.

122 Differential SALL4 Immunoexpression in Epithelioid Sarcomas and Malignant Rhabdoid Tumors

A Yoshida, NAsano, A Kawai, A Nakazawa, H Kishimoto, R Kushima. National Cancer Center Hospital, Tokyo, Japan; National Center for Child Health and Development, Tokyo, Japan; Saitama Children's Medican Center, Saitama, Japan.

Background: Epithelioid sarcomas (ESs) and malignant rhabdoid tumors (MRTs) are distinctive malignant neoplasms with characteristic clinicopathologic features. However, these 2 tumors share some phenotypes such as epithelioid/rhabdoid cytology, expression of epithelial markers, and immunohistochemical loss of INI1. This differential can be particularly problematic when tumors with MRT-like histology present in adults, and ancillary diagnostic tools are needed to separate the 2 entities. CD34 expression is widely believed to favor the diagnosis of ES, but no formal comparative study has been performed. Two recent reports showing SALL4 expression in $>80 \%$ of MRTs led us to evaluate the utility of this marker in differentiating ES and MRT.

Design: Archived materials of ESs $(N=34)$ and MRTs $(N=13)$ were retrieved and all diagnoses were confirmed. ESs were of distal $(\mathrm{N}=19)$, proximal $(\mathrm{N}=10)$, or hybrid $(\mathrm{N}=5)$ type, and mainly occurred in adult patients (age range, 15-54 y; median, 32 y). MRTs occurred in the CNS (atypical teratoid/rhabdoid tumors, $\mathrm{N}=4)$, kidney $(\mathrm{N}=$ 3 ), or other sites $(\mathrm{N}=6)$, and were in infants and children (age range, $0-12 \mathrm{y}$; median, 1 y). All ESs and MRTs lacked INI1 reactivity, except for 1 MRT case that retained
INI1 but lacked BRG1. In addition, 2 problematic cases were collected; these tumors occurred in adults and showed diffuse sheets of INI1-deficient uniform small round cells associated with scattered rhabdoid forms. A representative slide from each case was stained using antibodies against SALL4 and CD34, and the reactivity was scored as positive when $>1 \%$ of tumor cells were labeled.

Results: Eight (62\%) of 13 MRTs expressed SALL4, primarily in a focal manner (extent, $<5-90 \%$; median, $45 \%$ ) with variable intensity. In contrast, only $1(3 \%)$ distal variant of the 34 ES cases was SALL4 positive. CD34 staining was observed in $9(69 \%)$ of 13 MRTs and $24(70 \%)$ of 34 ESs. Both INI1-deficient problematic small round cell tumors in adults expressed SALL4.

Conclusions: Despite limited sensitivity and an often focal nature of reactivity, SALL4 expression can aid in distinguishing MRTs from ESs. Contrary to popular belief, CD34 was found to have no utility in making such distinctions. SALL4 expression in our 2 problematic cases may indicate that these tumors may be best classified as MRTs arising in adults.

123 CAMTA1 Immunostaining Is Not Useful in Differentiating Epithelioid Hemangioendothelioma from Its Potential Mimickers

Z Yusifli, K Kosemehmetoglu. Hacettepe University Faculty of Medicine, Ankara, Turkey. Background: Epithelioid hemangioendothelioma (EHE) is a rare member of vascular tumors of intermediate malignancy. CAMTA1 encodes a transcription factor in all multicellular organisms, conserved in Arabidopsis to humans and is known to be highly encountered in the memory related regions of the human brain. Recently, presence of $t(1 ; 3)$ translocation and WWTR1/CAMTA1 gene fusion, which enhances CAMTA1 expression, is found to be specific to EHE. In this study, we investigated the CAMTA1 immune expression profile of EHE and its potential mimickers using a commercially available CAMTA1 antibody immunohistochemically.

Design: Standard whole sections from the formalin fixed, paraffin embedded of 12 EHEs, 10 angiosarcomas, 9 epithelioid sarcomas, 8 malignant melanomas, 8 signet ring carcinomas, 7 lobular carcinomas of breast, 2 epithelioid mesotheliomas, 2 rhabdoid tumors and 12 micellaneous hemangiomas were immunostained for anti-CAMTA1 (ab64119, 1:200; Abcam) after pretreatment with citrate $\mathrm{pH} 6.0$ for 20 minutes at $97^{\circ} \mathrm{C}$. using Leica Bond detection kit with DAB chromogen. Nuclear CAMTA1 expression was scored for its intensity (as weak, moderate and strong) and extent as 'negative' ( $<5 \%$ of cells positive), ' +1 ' ( $5-25 \%$ of cells positive), ' $2+$ ' ( $25-50 \%$ of cells positive) and ' $3+$ ' ' $>50 \%$ of cells positive)

Results: Over $2+$ strong/moderate nuclear staining was seen in 63 out of 70 cases (90\%): $83 \%$ of EHEs, $90 \%$ of angiosarcomas, $89 \%$ of epithelioid sarcomas, $100 \%$ of malignant melanomas, $75 \%$ of signet ring carcinomas, $100 \%$ of lobular carcinomas of breast, $100 \%$ of epithelioid mesotheliomas, $50 \%$ of rhabdoid tumors and $92 \%$ of hemangiomas. Besides neurons, CAMTA1 expression was also observed in squamous epithelium, skin adnexa, breast lobules, prostate glands, bile ducts, colonic mucosa and gastric pits whereas hepatocytes, trophoblasts, lung, thyroid, smooth and striated muscles and fibroblasts were negative.

Conclusions: EHE, its potential morphological mimickers and other benign or malignant vascular tumors showed strong and diffuse CAMTA1 expression, nullifying the potential use of CAMTA1 immunohistochemical staining as an adjunct in the differential diagnosis of epithelioid EHE. CAMTA1 expression was observed throughout the body, especially epithelial compartments, including mucosae, mesothelium and endothelium.

\section{Giant Cell Tumor of Bone in Children and Adolescent -A Series} of 43 Cases

Y Zhang, TW Bauer, JB Wojcik, GP Nielsen, AE Rosenberg. Cleveland Clinic, Cleveland, $\mathrm{OH}$; Massachusetts General Hospital, Boston, MA; University of Miami, Miami, FL. Background: Giant cell tumor of bone (GCT) is a benign, locally aggressive neoplasm, which most frequently involves the epiphyseal ends of long bones in skeletally mature individuals. It rarely arises in individuals younger than 18 years of age. The aim of this study is to examine the clinical and pathologic characteristics of giant cell tumor of bone in children and adolescents.

Design: The study cohort was identified from the authors consult files and from the surgical pathology files of the participating institutions. The clinical, radiological and pathological features of the tumors were analyzed.

Results: 43 patients ( 26 females and 17 males, with median age 14.8 years, ranging from 8 to 18) were identified. Pain was the most common symptom, followed by swelling and pathologic fracture. As in adults, most tumors arose in the ends of long bones, with the femur as the most common site (10), followed by the tibia (9). The tumors also often developed in the small bones of the hands and feet (8), and other sites included the vertebral body (4), pubic ramus (3), fibula (3), sacrum (2), humerus (1), ulna (1), olecranon (1), and maxilla (1). Radiographically, GCT in long bones often manifested as an eccentric, lytic, circumscribed, expansile mass involving the epiphyseal-metaphyseal area. In the short or flat bones, the tumor often completely replaced the underlying bone. On magnetic imaging studies the tumors were typically hypointense on T1 and hyperintense on T2 weighted images. Soft tissue components were common. Microscopically, most tumors had the classic features of GCT. Atypical features included vascular invasion, increased mitotic activity, and focal cytologic atypia. Patients were usually treated with curettage and bone grafting. Follow-up information available on 8 patients ( 22 months to 26 years, av. 10 yrs.) showed that they were all disease free after treatment. Three cases involving the pubic ramus showed the most aggressive features, and one of the cases underwent malignant transformation after multiple recurrences.

Conclusions: While it is rare, GCT occurs in children and adolescents with a female predominance, and a predilection for the ends of major tubular bones $(53.5 \%)$. In comparison to adults it affects the small bones of the hands and feet more frequently $(18.6 \%)$. Curettage is the mainstay of therapy and patients usually have a good outcome. 